\title{
Phase Transition and Metastability in a Stochastic System of Spiking Neurons
}

\author{
Morgan André \\ TESE APRESENTADA \\ AO \\ Instituto DE Matemática e Estatística \\ DA \\ Universidade De SÃo PaUlo \\ PARA \\ OBTENÇÃO DO TÍTULO \\ $\mathrm{DE}$ \\ DOUTOR EM CIÊNCIAS \\ Programa: Estatística \\ Orientador: Prof. Dr. Antonio Galves
}

This thesis was produced as part of the activities of FAPESP Research, Innovation and

Dissemination Center for Neuromathematics (grant number 2013/07699-0, S. Paulo Research Foundation), and the author was supported by a FAPESP scholarship (grant number 2017/02035-7).

São Paulo, setembro de 2020 


\section{Phase Transition and Metastability in a Stochastic System of Spiking Neurons}

Esta versão da tese contém as correções e alterações sugeridas pela Comissão Julgadora durante a defesa da versão original do trabalho, realizada em 13/11/2020. Uma cópia da versão original está disponível no Instituto de Matemática e Estatística da Universidade de São Paulo.

Comissão Julgadora:

- Prof. Dr. Jefferson Antonio Galves (orientador) - IME-USP

- Prof. Dr. Pablo Augusto Ferrari - IME-USP

- Prof ${ }^{a}$. Dr ${ }^{a}$. Eva Löcherbach - CY Cergy Paris Université

- Prof. Dr. Christophe Pouzat - Université de Paris

- Prof ${ }^{a}$. Dra ${ }^{\text {a }}$ Patricia Reynaud-Bouret - Université Côte d'Azur 


\section{Acknowledgement}

First of all I would like to express my gratefulness to Christophe Pouzat and Antonio Galves. Thanks to Christophe for introducing me to the world of research, to the fascinating mathematical problems which arise in the field of neurosciences, and thanks for your inspiring and unexampled attention to details and sense of scientific pragmatism. Thanks also for having me introduced to the NeuroMat team. Thanks to Antonio, for having given to me the opportunity to be part of this team, and for having shared with me during these three years his inextinguishable enthousiasm, his wide knowledge as well as his sense of humor.

Thanks a lot to all the administrative staff, especially to Lourdes Netto, whose legendary efficiency have been of great helps whenever I had trouble with the sometimes tricky Brazilian bureaucracy. Thanks to Elisangela from the international office, who has been of great help as well. Thanks you to all the researchers of the lab, past and present, some of which are now my friends as well as they are my colleagues. Thanks to Guillerme, Aline, Noslen and Ioannis, with whom I have learned as much about mathematics as I have about samba and São Paulo's bars and restaurants. I would also like to thank my friend Léo, who is responsible for at least half of the proof of the main result of Chapter 5, for having been such a stimulating co-author. I thank Fernando and Cecilia for having been stimulating co-authors as well. I am also in debt to Antonio Marcos for insightful discussions, and for the useful references he indicated me while we were working on similar subjects. More generally I would like to thank all the members of NeuroMat here and abroad, who make it such an exciting project to be part of, and who are too numerous for me to list them.

Thanks also to the professors and researchers of the institute of mathematics and statistics of USP for all the instructive lectures and conferences I had the occasion to attend and thanks to the FAPESP for the financial support.

Finally thanks to my beloved Laurie, as well as my old friend Antoine, for having visited me many times in São Paulo along these years. Thanks also to my friend Alexandre for the innumerous transatlantic discussions and chess games we had during my stay in Brazil, whenever I would take a break from my (otherwise studious) mathematical investigations. 


\section{Abstract}

We study a continuous-time stochastic system of spiking neurons from the perspective of phase transition and metastability, using mathematical concepts and techniques borrowed from statistical physics.

The model consists in a finite or countable set of neurons $S$, each of them being associated to a point process modeling its spiking activity. At any time, a neuron either spike at rate one or doesn't spike depending on whether its membrane potential is positive or null. The propagation of a spike is done through chemical synapse, meaning that the membrane potential of the spiking neuron is reset to 0 at the moment of the spike, while the membrane potential of its post-synaptic neurons is increased by one unit. Moreover, each neuron is subject to a phenomenon known as leakage, which is represented as a point process of some parameter $\gamma$. At any of the atoms of this point process, the membrane potential of the neuron concerned is immediately reset to 0 .

What we obtain is a system of interacting point processes, and as the neurons can only be in two possible states (active or quiescent, depending on whether the membrane potential is positive or null), it can be seen as an interacting particles system, i.e. a Markov process taking value in $\{0,1\}^{S}$.

It has already been proven that this model exhibits a phase transition phenomenon with respect to the leakage parameter when the graph of interaction is the one-dimensional lattice (see [FGGL18]), that is $S=\mathbb{Z}$ with nearest neighbors interaction. Namely, there exists a critical value $\gamma_{c}$ such that the process dies almost surely when $\gamma>\gamma_{c}$, or survives for an infinite amount of time if $\gamma<\gamma_{c}$. Here we prove that, in this one-dimensional lattice setting, at least in a portion of the sub-critical region, the process exhibits a metastable behavior. What we mean by this is that the renormalized time of extinction of a finite version of the process is asymptotically memory-less with respect to the number of neurons. We also prove that this result doesn't hold when $\gamma>1$, and that in this case the renormalized time of extinction is actually asymptotically deterministic. We also show that the result of phase transition can be extended to the $d$-dimensional lattice, that is $S=\mathbb{Z}^{d}$ with nearest neighbors interaction. Finally we turn ourselves to the process in which the graph of interaction is the complete graph (everybody interact with everybody), and we show that the result of metastability holds for any $\gamma>0$ in this case.

Keywords: Metastability, Phase transition, Biological neural networks stochastic models, Interacting particle systems. 


\section{Resumo}

Nessa tese, estudamos um sistema estocástico em tempo continuo de neurônios gerando disparos, do ponto de vista dos fenômenos de transição de fase e de metaestabilidade, usando conceitos matemáticos e técnicas emprestado da física estatística.

O modelo consiste em um conjunto $S$ finito o enumerável de neurônios, cada um associado com um processo pontual representando os disparos. A cada tempo, um neurônio pode o emitir disparos com uma intensidade de 1 o não emitir nenhum disparo, dependendo do valor do potencial de membrana (positivo o zero). A propagação dos disparos ocorre através de sinapses químicas, o que significa que o potencial de membrana do neurônio disparando perde o seu potencial de membrana, e que o potencial de membrana dos neurônios post-sinápticos aumenta de uma unidade. Além disso, o potencial de membrana de cada neurônio e afetado por um fenômeno de natural vazamento, o qual e representado com um processo pontual de taxa $\gamma$. A cada átomo desse processo o neurônio perde o potencial de membrana dele sem influenciar os neurônios post-sinápticos.

O que nos obtemos é um sistema de processos pontuais em interação, e tendo em vista que cada neurônio so pode ter dois estados diferente (ativado ou em repouso), ele pode ser visto como um processo de partículas em interação, ou seja um processo Markoviano tendo valores em $\{0,1\}^{S}$.

Foi provado que, no caso no qual o grafo de interação é uma linha, ou seja $S=\mathbb{Z}$ com interação de tipo vizinho mais próximo (veja [FGGL18]), esse modelo está sujeito a uma transição de fase em relação ao parametro $\gamma$. Isto é, existe um valor critico $\gamma_{c}$ tal que o processo morre quase certamente quando $\gamma>\gamma_{c}$, o sobrevive por um tempo infinito quando $\gamma<\gamma_{c}$. Nessa tese nos mostramos que, no caso em qual o grafo de interação é a linha, pelo menos numa porcão da região subcrítica, o processo apresenta um comportamento metaestável. Isso significa que o tempo de extinção renormalizado de uma versão finita do processo é assimptoticamente sem memoria em relação ao numero de neurônios. Nos provamos também que não é o caso quando $\gamma>1$, e que na verdade o tempo de extinção renormalizado e assimptoticamente determinístico nesse caso. Nos generalizamos também o resultado de transição de fase para o caso $S=\mathbb{Z}^{d}$ com interação de tipo vizinhos mais próximos. Em fim, nos consideramos o processo no qual o grafo de interação é o grafo completo (todo mundo interage com todo mundo), e nos mostramos que nesse caso o resultado de metaestabilidade vale para todo $\gamma>0$.

Palavras-chave: Metaestabilidade, Transição de fase, Sistemas de partículas em interação, Modelos estocásticos de redes de neurônios biológicos. 


\section{Content}

$\begin{array}{ll}\text { Frequently Used Notation } & \text { ix }\end{array}$

List of Figures $\quad$ xi

1 Introduction $\quad 1$

1.1 Biological neural networks . . . . . . . . . . . . . . . . 1

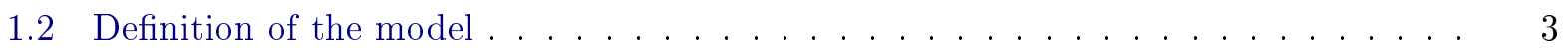

1.3 Phase transition and metastability in biological neural networks . . . . . . . . . . 4

1.4 Results presented in this thesis $\ldots \ldots \ldots \ldots \ldots \ldots$

2 Phase transition in the lattice $\quad 11$

2.1 Phase transition in the one-dimensional lattice and generalization to the d-dimensional lattice ................................ 11

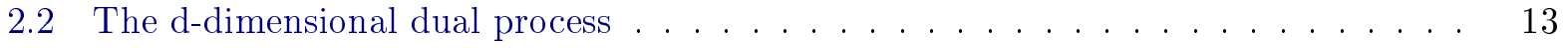

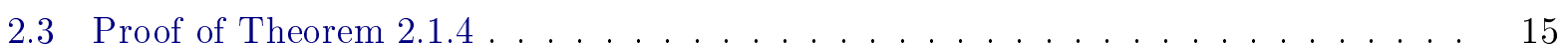

3 Metastability in the sub-critical region for the one-dimensional lattice process 17

3.1 Definitions and result . . . . . . . . . . . . . . . . . . . . 17

3.2 The graphical construction . . . . . . . . . . . . . . . . . . . . 19

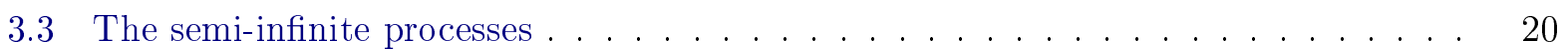

3.4 The dual process . . . . . . . . . . . . . . . . . . 22

3.4.1 Formal definition of the dual process . . . . . . . . . . . . . . . 22

3.4.2 Graphical construction of the dual process . . . . . . . . . . . . . . 23

3.5 Asymptotic behavior . . . . . . . . . . . . . . . . . . . 23

3.5.1 Asymptotic behavior of the infinite processes . . . . . . . . . . . 23

3.5.2 Asymptotic behavior of the semi-infinite processes . . . . . . . . . . 29

3.6 Some technical lemmas . . . . . . . . . . . . . . . . . . . . . 35

3.7 Proof of Theorem $3.1 .1 \ldots \ldots \ldots \ldots \ldots \ldots$

4 Asymptotically deterministic time of extinction in the super-critical region for $\begin{array}{ll}\text { the one-dimensional lattice process } & 47\end{array}$

4.1 Asymptotically deterministic time of extinction . . . . . . . . . . . . 47

4.2 Proof of Theorem $4.1 .1 \ldots \ldots \ldots \ldots \ldots \ldots$

4.2 .1 Proof of Proposition $4.1 .2 \ldots \ldots \ldots \ldots \ldots \ldots$

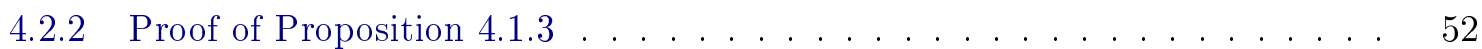


$5 \quad$ The complete graph process $\quad 55$

5.1 Metastability in the complete graph process . . . . . . . . . . . . 55

5.2 Proof of Theorem $5.1 .1 \ldots \ldots \ldots \ldots \ldots \ldots$

6 Conclusion and perspectives $\quad 65$

$\begin{array}{lll}\text { A } & \text { Theory of duality } & 67\end{array}$

$\begin{array}{ll}\text { B Some usefull results } & 69\end{array}$

B.1 A measure determining set of functions . . . . . . . . . . . . . . . . 69

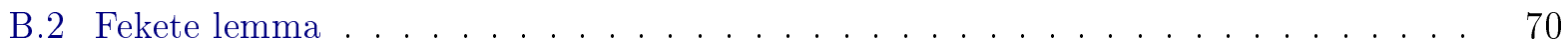

B.3 Continuous time branching process $\ldots \ldots \ldots \ldots \ldots \ldots \ldots \ldots \ldots$

$\begin{array}{ll}\text { Bibliography } & 73\end{array}$ 


\title{
Frequently Used Notation
}

\author{
$\mathbb{Z} \quad$ The one-dimensional integer lattice \\ $\mathbb{Z}^{d} \quad$ The d-dimensional integer lattice. \\ $\mathbb{N} \quad$ The set of the non-negative integers. \\ $\mathbb{R} \quad$ The set of the real numbers. \\ $\llbracket-N, N \rrbracket \quad$ The set of integers $\mathbb{Z} \cap[-N, N]$. \\ $S \quad$ A finite or countable set representing neurons (or sites). \\ $\mathscr{P}(S) \quad$ The class of all subsets of $S$. \\ $A, B \quad$ Typical elements of $\mathscr{P}(S)$ \\ $\{0,1\}^{S} \quad$ The set of configurations of the process, i.e. sequences of 0 and 1 indexed by $S$. \\ $\xi, \eta, \zeta \quad$ Typical elements of $\{0,1\}^{S}$. \\ $\xi \leq \eta \quad$ Partial order relation between configurations. \\ $\xi \equiv 0 \quad$ The "all zero" configuration. \\ $\xi \equiv 1 \quad$ The "all one" configuration. \\ $(\xi(t))_{t \geq 0} \quad$ The Markov Process which represents the system in the one-dimensional lattice \\ setting. \\ $(\widetilde{\xi}(t))_{t \geq 0} \quad$ The dual of the process $(\xi(t))_{t \geq 0}$. \\ $\left(\xi_{N}(t)\right)_{t \geq 0} \quad$ The finite version of $(\xi(t))_{t \geq 0}$. \\ $(\eta(t))_{t \geq 0} \quad$ The Markov Process which represents the system in the d-dimensional lattice \\ setting. \\ $(\widetilde{\eta}(t))_{t \geq 0} \quad$ The dual of the process $(\eta(t))_{t \geq 0}$. \\ $\left(\eta_{N}(t)\right)_{t \geq 0} \quad$ The finite version of $(\eta(t))_{t \geq 0}$. \\ $\left(\zeta_{N}(t)\right)_{t \geq 0} \quad$ The Markov Process which represents the system in the complete graph fo size \\ $N$ setting. \\ $\tau, \kappa \quad$ The times of extinction of the processes in the lattice setting. \\ $\tilde{\tau}, \tilde{\kappa} \quad$ The times of extinction of their dual. \\ $\tau_{N}, \sigma_{N} \quad$ The time of extinction of the finite versions of $(\xi(t))_{t \geq 0}$ and $(\zeta(t))_{t \geq 0}$ respec- \\ tively. \\ $\mu, \nu \quad$ Probability measures on $\{0,1\}^{S}$ (or equivalently on $\mathscr{P}(S)$ ). \\ $\mu \leq \nu \quad$ Partial order relation between probability measures. \\ $\delta_{\emptyset} \quad$ The probability measure putting the whole mass on $\xi \equiv 0$ (or equivalently on \\ $\emptyset)$. \\ $\mathscr{I} \quad$ The set of invariant measures for $(\xi(t))_{t \geq 0}$. \\ $\mathscr{I}_{e} \quad$ The extremal elements of $\mathscr{I}$. \\ $Y_{n} \underset{n \rightarrow \infty}{\stackrel{\mathscr{D}}{\longrightarrow}} Y$. The sequence $\left(Y_{n}\right)_{n \geq 0}$ converges in distribution toward $Y$. \\ $Y_{n} \underset{n \rightarrow \infty}{\stackrel{\mathbb{P}}{\rightarrow}} Y$. The sequence $\left(Y_{n}\right)_{n \geq 0}$ converges in probability toward $Y$. \\ $\mathscr{E}(1) \quad$ An exponentially distributed random variable of mean 1.
}




\section{List of Figures}

1.1 The figure showing the different type of neuronal responses in the original Fuster's

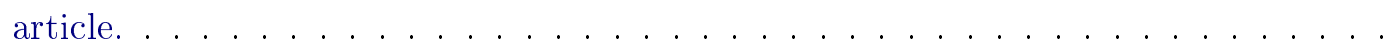

1.2 Simulation of the model with $\gamma=2$ for a system with 100 neurons. On the top left figure the histogram of the renormalized time of extinction is plotted. The histogram itself is normalized so that the area under the curve is equal to one. On the top right figure, the survival function is plotted (in blue). The red curve is the graph of $t \mapsto e^{-t}$ in both plot. Both are constructed on the same data set, which consists on 3000 simulation of the process. One can observe on the histogram and the survival function that the data are not exponentially distributed at all, instead the histogram seems to have its values concentrated around 1 . On the bottom figure a typical trajectory of the process is plotted. One can observe that the number of active neurons constantly decreases and rapidly reaches 0 . No metastability can be seen in these figures. . . .

1.3 Simulation of the model with $\gamma=0.35$ for a system with 100 neurons. On the top left figure the histogram of the renormalized time of extinction is plotted. The histogram itself is normalized so that the area under the curve is equal to one. On the top right figure, the survival function is plotted (in blue). The red curve is the graph of $t \mapsto e^{-t}$ in both plot. Both are constructed on the same data set, which consists on 3000 simulations of the process. One can observe that the data are well approximated by an exponential distribution, as a matter of fact, the survival function is almost indistinguishable from the exponential function. On the bottom figure a typical trajectory of the process is plotted. One can observe that after a rapid decrease the number of active neurons stabilizes in pseudo-stationary state and oscillates before finally dying because of a sequence of abnormally close leaks. This behavior is typical of a metastable dynamic. . . . . . . . . . . . .

3.1 A realization of the random graph $\mathscr{G}$. In blue all the possible valid paths starting from $(0,0)$. Here the configuration of the process at time $t$ when the initial configuration is the singleton $\{0\}$ is the set $\{-4,-2,1\} \ldots \ldots \ldots \ldots$

3.2 A realization of the dual random graph $\mathscr{G}^{\prime}$. In blue all the possible dual-valid paths starting from $(0,0)$. Here the configuration of the dual process at time $t$ when the initial configuration is the singleton $\{0\}$ is the set $\{-1,1,2\} \ldots \ldots$

3.3 Example of a possible contour with $n=7$. Some of the direction vectors are explicitly

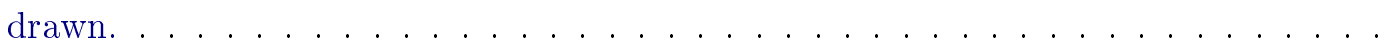

3.4 On the left side we can see $F_{j}^{\dagger}$ and $F_{j}$ that are represented on the time-space diagram. The $\delta$ corresponds to $F_{j}^{\dagger}$ and the double arrow corresponds to $F_{j} . G_{j}^{\dagger}$ and $G_{j}$ are represented on the right side. The red line represents $\Gamma$ and the dashed part of it represents $D_{j}$, which took value $d l d$ on the left, and $u l u$ on the right. . . . . . . 
4.1 The construction of the current section, represented in the space-time diagram introduced in the previous chapter. the horizontal axis represents the lattice $\mathbb{Z}$ and the vertical axis represents the time. The blue points are the elements of the configuration $A_{N}$, while the blue lines represent the valid paths in the graphical construction in a realization of the modified process $\left(\bar{\xi}_{N}^{0}(t)\right)_{t \geq 0}$, starting from these points. The vertical gray lines represents the particles that are not allowed to spike in our construction, acting as hermetic walls in the space-time diagram. The green area represent the portion of the diagram in which the event $E_{t}$ is satisfied. . . . . . . . .

5.1 The transition diagram of the Markov jump process $\left(\widetilde{\zeta}_{N}(t)\right)_{t \geq 0} \ldots \ldots \ldots \ldots$ 


\section{Chapter 1}

\section{Introduction}

\subsection{Biological neural networks}

Biological neural systems are networks containing a typically huge number of components, the neurons. A neuron is a cell whose membrane includes voltage-gated ionic channels that allow fluctuation of the concentrations of ions inside and outside of the cell body, creating a difference of potential called the membrane potential. The evolution of the membrane potential of a neuron over time is punctuated by sudden depolarizations, called action potentials or spikes, which tend to happen when the membrane potential is high enough. These spikes are propagated in the network via chemical synapses. Indeed after the occurrence of a spike, neurotransmitters are released into the synaptic clefts between the neuron that emitted the spike and its postsynaptic neurons, leading to an increase or a decrease in membrane potential for these postsynaptic neurons depending on whether the synapse is excitatory or inhibitory. In the excitatory case, a spike of the presynaptic neuron thus results in an increase of the spiking probability for the postsynaptic neurons. After a spike has occurred, the membrane potential of the neuron that just spiked is always reset to the same resting value, inducing a phenomenon of memory-loss: for a given neuron, the previous spikes of its presynaptic neurons has no influence anymore once it has spiked itself. Moreover, in between spikes the membrane potential tends to decrease as it is affected by the natural diffusion of ions that occurs through the membrane when some equilibrium has not been reach in the cell, a phenomenon known as leakage. It induces a smoother memory-loss effect, due to the fact that if a presynaptic spike has occurred a long time ago, even if the postsynaptic neuron has not spiked up to the current time, the resulting increase in membrane potential will eventually be small or even null. We refer to [GK77] for a detailed account on these subjects.

The mathematical modeling of biological neurons and biological neural networks has now a pretty long history, starting in 1907 with the classical integrate-and-fire model proposed by the french neuroscientist Louis Lapicque, which model the membrane potential of a single neuron by means of a simple differential equation. In 1952, Hodgkin and Huxley proposed the so-called Hogkin-Huxley model, which describes in greater details the membrane potential dynamic using a set of nonlinear differential equations. A wide variety of improvements or alternative models have been then introduced using deterministic models mostly based on 
differential equations.

Then, as more evidence arose during the second half of the twentieth century that biological neural networks behave in an inherently stochastic manner, and likely as a result of the apparition and diffusion of the modern theory of probability as well, stochastic models started to be increasingly considered. We refer the reader to [RD10] for a recent review on the neuroscientific aspects of this matter. One of the most relevant class of stochastic models in continuous-time is the one that make use of point processes. It is indeed now well accepted that spikes are the unitary elements by which biological neural networks encode information and transmit signals, so that in order to understand how the brain work functionally, it is relevant to focus on these spikes understood as punctual events. Some example of point-processes used in theoretical neuroscience are Hawkes processes [HAW71, CCMDRB15, CSK88, HRBR15] and Wold processes [KV01].

The model we consider in this thesis takes it roots in [GL13]. This last article introduced a stochastic model now known as the Galves-Löcherbach model in the literature, or simply GL model. It was originally a discrete time stochastic process with an infinite number of interacting components, where each neuron can be in state 1 or 0 at any time $t \in \mathbb{N}$, depending on whether it spikes or not at this time. In this model we have a countable set of neurons $I$, and if $X_{i}(t)$ denotes the state of neuron $i \in I$ at time $t$, then the probability for a a given neuron to be spiking at time $t \in \mathbb{N}$ is given by

$$
\mathbb{P}\left(X_{i}(t)=1 \mid \mathscr{F}_{t-1}\right)=\phi_{i}\left(\sum_{j \in I} W_{j \rightarrow i} \sum_{s=L_{t}^{i}}^{t-1} g_{j}(t-s) X_{s}(j), t-L_{t}^{i}\right),
$$

where $W_{j \rightarrow i}$ denotes the synaptic weight describing the influence of neuron $j$ on neuron $i$, $g_{j}$ is the function modeling the leakage, $L_{t}^{i}$ is the time of the last spike affecting neuron $i$ up to time $t, \mathscr{F}_{t-1}$ is the standard filtration associated to the process up to time $t$, and $\phi_{i}: \mathbb{R} \times \mathbb{N} \rightarrow[0,1]$ is an activation function associated to neuron $i$.

This model is informally speaking a stochastic version of the leaky integrate and fire model. Since this original paper, other versions of this model has been studied, see for example [GEP19, MGLP15, DO14, FL16]. We refer to [GL15] for a general review.

The model we consider in this thesis is both a simplification and an extension of this original model. It was introduced in [FGGL18], and it is a continuous-time model. For each neuron, the spiking times are represented by a point process. The leakage, unlike in the original model, occurs at discrete times and is represented by a point process as well. This is mathematically convenient as it allows us to see our process as an interacting particle system (see [LIG85]), and to prove results that we believe are out of reach in the original model. At any of the leak times the membrane potential is reset to the resting value, which is 0 by convention. Moreover, like in the original model, the membrane potential of any given neuron is also reset to 0 whenever this neuron spikes. This implies that the time evolution of each single neuron has a memory of variable length, which is an interesting feature both from a mathematical and a biological point of view. In the original setting considered in [FGGL18] there is a countably infinite number of neurons, which is not unreasonable given that the human brain has a number of neurons of the order of $10^{11}$. In this thesis we will consider both finite and infinite systems. Moreover this model assume that all the neurons 
are excitatory with respect to their postsynaptic neurons, with the same synaptic weight, conventionally fixed to 1 . We give a formal definition of the model in the next section.

\subsection{Definition of the model}

The model considered in this work is as follows. $S$ is a finite or countable set representing the neurons, and to each $i \in S$ is associated a set $\mathbb{V}_{i}$ of presynaptic neurons. If you consider the elements of $S$ as vertices, and draw and edge from $i$ to $j$ whenever $i \in \mathbb{V}_{j}$, that is if you define $E=\left\{(i, j): i \in \mathbb{V}_{j}\right\}$, then you obtain the graph of the network $G=(S, E)$. Each neuron $i$ has a membrane potential evolving over time, represented by a stochastic process which takes its values in the set $\mathbb{N}$ of non-negative integers and which is denoted $\left(X_{i}(t)\right)_{t \geq 0}$. Moreover each neuron is also associated with a Poisson process $\left(N_{i}^{\dagger}(t)\right)_{t \geq 0}$ of some parameter $\gamma$, representing the leak times. At any of these leak times the membrane potential of the neuron concerned is reset to 0 . Finally an other point process $\left(N_{i}(t)\right)_{t \geq 0}$ representing the spiking times is also associated to each neuron, which rate at time $t$ is given by $\phi\left(X_{i}(t)\right), \phi$ being some function called the activation function. When a neuron spikes its membrane potential is reset to 0 and the membrane potential of all of its postsynaptic neurons is increased by one (that is the neurons of the set $\left\{j: i \in \mathbb{V}_{j}\right\}$ ). All the point processes involved are assumed to be mutually independent. More formally, beside asking that $\left(N_{i}^{\dagger}(t)\right)_{t \geq 0}$ be a Poisson process of some parameter $\gamma$, this is the same as saying that $\left(N_{i}(t)\right)_{t \geq 0}$ is the point process characterized by the following equations

$$
\mathbb{E}\left(N_{i}(t)-N_{i}(s) \mid \mathscr{F}_{s}\right)=\int_{s}^{t} \mathbb{E}\left(\phi\left(X_{i}(u)\right) \mid \mathscr{F}_{s}\right) d u
$$

where

$$
X_{i}(t)=\sum_{j \in \mathbb{V}_{i}} \int_{l L_{i}(t), t[} d N_{j}(s),
$$

$L_{i}(t)$ being the time of the last event affecting neuron $i$ before time $t$, that is,

$$
L_{i}(t) \stackrel{\text { def }}{=} \sup \left\{s \leq t: N_{i}(\{s\})=1 \text { or } N_{i}^{\dagger}(\{s\})=1\right\} .
$$

$\left(\mathscr{F}_{t}\right)_{t \geq 0}$ is the standard filtration for the point processes involved here, that is the filtration which at any time $t \geq 0$ is equal to the $\sigma$-algebra generated by the family $\left\{N_{i}(s), N_{i}^{\dagger}(s), s \leq\right.$ $t, i \in S\}$.

In the present work the function $\phi$ will always be the hard-threshold function defined for any $x \in \mathbb{R}$ by $\phi(x)=\mathbb{1}_{x>0}$. For any fixed $t \geq 0$ and $i \in S$ the quantity of interest is then given by $\mathbb{1}_{X_{i}(t)>0}$, as it corresponds to the infinitesimal spiking rate of neuron $i$ at time $t$. Depending on whether $\mathbb{1}_{X_{i}(t)>0}$ is equal to 1 or 0 we will say that neuron $i$ is active or quiescent respectively. The dynamic described in the first paragraph of this section can then be reformulated as follows. Whenever a spike occurs, the neuron that spiked becomes quiescent, as can be seen from equations (1.3) and (1.2), while its postsynaptic neurons become active if they weren't already, as can be seen from equation (1.2). The choice of the hard-threshold for the function $\phi$ has however no consequence on the leak times, which 
effect of getting any active neuron to the quiescent state result from equations (1.3) and (1.2). If for any $i \in S$ and $t \geq 0$ we write $\eta_{i}(t)=\mathbb{1}_{X_{i}(t)>0}$, and $\eta(t)=\left(\eta_{j}(t)\right)_{j \in S}$, then the resulting process $(\eta(t))_{t>0}$ is an interacting particle system, that is, a Markovian process taking value in $\{0,1\}^{S}$ (see [LIG85]). His infinitesimal generator (see [FGGL18]) is given by

$$
\mathscr{L} f(\eta)=\gamma \sum_{i \in S}\left(f\left(\pi_{i}^{\dagger}(\eta)\right)-f(\eta)\right)+\sum_{i \in S} \eta_{i}\left(f\left(\pi_{i}(\eta)\right)-f(\eta)\right)
$$

where $f:\{0,1\}^{S} \mapsto \mathbb{R}$ is a cylinder function ${ }^{1}$, and the $\pi_{i}^{\dagger}$ 's and $\pi_{i}$ 's are maps from $\{0,1\}^{S}$ to $\{0,1\}^{S}$ defined for any $i \in S$ as follows:

$$
\left(\pi_{i}^{\dagger}(\eta)\right)_{j}= \begin{cases}0 & \text { if } j=i \\ \eta_{j} & \text { otherwise }\end{cases}
$$

and

$$
\left(\pi_{i}(\eta)\right)_{j}= \begin{cases}0 & \text { if } j=i \\ \max \left(\eta_{i}, \eta_{j}\right) & \text { if } i \in \mathbb{V}_{j} \\ \eta_{j} & \text { otherwise. }\end{cases}
$$

The $\pi_{i}^{\dagger}$ 's correspond to the leakage effect mentioned above, and the $\pi_{i}$ 's correspond to the spikes. Of course the graph of interaction needs to satisfy some conditions of regularity for this infinitesimal generator to even make sense, but the instantiations that we consider in this article are simple enough to trivially satisfy these conditions. A simple way of reading the generator above is the following. Whenever a neuron is active, it is associated with two independent exponential random clocks of parameter 1 and $\gamma$, and it spikes or leaks respectively depending on which one rings first.

\subsection{Phase transition and metastability in biological neural networks}

Three crucial and interconnected areas of research in the study of biological neural networks are the question of phase transition, criticality and metastability. See [WER07] for a global neuroscientific survey on the subject. These concept originally come from physics, and specifically from statistical physics and particle physics. The expression of "phase transition" refers to the property of a complex system, typically a dynamical system or, in our case, a stochastic process, to present two or more qualitatively different behaviors depending on the specific values of the parameters that are chosen. In a stochastic context it typically means that a process suddenly changes from one invariant measure to another as you run through the parameter space. It was shown for example in [FGGL18] that the stochastic process defined in the previous section is subject to a phase transition with respect to the leakage parameter $\gamma$, when the graph $G$ is the one-dimensional lattice $\mathbb{Z}$ with nearest neigh-

\footnotetext{
${ }^{1}$ A cylinder function is a function on $\{0,1\}^{S}$ which depends only on a finite number of sites in $S$.
} 
bor interaction. Namely, there exists a value $\gamma_{c}$ such that the process continues spiking infinitely many time with positive probability if $\gamma<\gamma_{c}$, or stop spiking almost surely if $\gamma>\gamma_{c}$.

When a system is subject to a phase transition, the value at which the shift is made is then said to be a critical value. These critical values are of primary interest as it has been proposed in the literature that in the brain, as well as in other physical systems, the information transmission is maximized at the critical values (see [BAK96, BP03]), while some other authors argue that it might be maximized for slightly super-critical or slightly sub-critical values (see [CGL17]).

The concept of metastability on the other hand can be seen as some kind of dynamical phase transition. Ranging from avalanche to supercooling water and energetic states of atomic nucleus, the adjective "metastable" is used in various fields of knowledge to qualify a state (or a collection of states) which is seemingly stable in the system in which it appears, but such that a tiny perturbation can push the system toward an even more stable state. By extension we often say that the system itself is metastable or subject to metastability, which is the meaning in which the expression will be used mostly in this thesis.

In statistical physics, a metastable dynamic evolves in a pseudo-stationary manner for an unpredictably long time, before falling into the actual equilibrium because of an infinitesimally rare but macroscopically unavoidable deviation from this pseudo-stationary phase. The perturbation here is statistical and inherent to the system, or endogenous, as it consists in the occurrence of a rare event caused by the dynamic of the system itself. This concept has now a long history in statistical physics as well as in probability theory. In a seminal paper (see [LP71]) O. Penrose and J. L. Lebowitz proposed the following characterization for a metastable thermodynamic state :

1. only one thermodynamic phase is present,

2. a system that starts in this state is likely to take a long time to get out,

3. once the system has gotten out, it is unlikely to return.

The first and third points will be easily treated in our model as we will consider finite systems in which the spiking activity will stop almost surely, so that there will be only one invariant measure (one phase), which is the one putting the whole mass on the state where all the neurons have a null membrane potential, which is a trap for the system. About ten years latter, M. Cassandro, A. Galves, E. Olivieri and M. E. Vares introduced in [CGOV84] a refinement of the second point, as they realized that a crucial characteristic of a wide variety of metastable stochastic dynamics is not only that the exit time from the metastable state is long, but that it is also in some sense unpredictable, which translate mathematically by saying that it is exponentially distributed (asymptotically). The reason for this is that the exponential distribution is characterized by the memory-less property: knowing that the system survived up to time $t$ gives you no information about what should happen next. From that point this property has been studied in a wide variety of stochastic dynamics. See for example [SCH85], [MOU93] or [FMNS15] (as a non-exhaustive list of references). Since then alternative approaches has also been developed and we refer to [OV05] for a complete review. 
Moreover the concept of metastability has become increasingly important in the neuroscientific literature during the last decades. It usually refers to the brain ability to switch periodically and spontaneously between different functional networks (see for example [DKJR17]), and it is considered by many to be one of the fundamental tools the brain has to its disposition to process information coming from the noisy, complex and constantly changing environment in which living beings usually evolve.

One of the first work to highlight such phenomenon is maybe the article written by J.M. Fuster in 1973 [FUS73]. It relates an experiment involving recordings of the neuronal activity in the prefrontal cortex of adult monkeys via a set of microelectrodes during a delayed-response test. Schematically the experiment is as follows: an adult male monkey (macaca mulatta) is placed in front of a setting involving two food wells protected by a window, one of the food well being empty and the other one containing a piece of apple. In the first period, called the "cue period", which last 7 seconds, the setting is simply presented to the monkey and, just before the end of this period, the two wells are covered by two pieces of wood. The window is them covered in order to prevent the monkey from seeing the setting, and the second period, called the "delay period", starts. After a duration of 18 seconds the delay period ends, the window is uncovered and the animal is given the choice of one of the two objects, which he can now accesses through unlocked doors below the screen. Microelectrodes are set in various places of the prefrontal cortex of the monkey, and the spiking activity during the three periods are retrieved in the form of spike trains. After that, Fuster was able to classify all unitary recordings (328 in total) into six categories of neuronal responses. The two most interesting categories for us are the one he called type $\mathrm{C}$ and type $\mathrm{D}$. They differ by the fact that type $\mathrm{C}$ presents an increased activity during the cue period while type $\mathrm{D}$ presents an inhibited activity during the same period, but more interestingly they resemble each other as they both exhibit a sustained activity during all the delay period, significantly higher than the baseline activity observed between successive experiments, which only resolves at the end of this delay period. Moreover, in a second experiment in which the monkeys were purposely distracted by visual and auditory stimuli during the delay period, these type $\mathrm{C}$ and type $\mathrm{D}$ responses were shown to be the ones that are the most intensively impacted by these perturbations, giving evidences that these are the types of neuronal responses that are the most likely to be linked with short-term memory. While the expression "metastability" is never used in this paper as the subject was not so widely studied at that time, this kind of phenomenon is clearly reminiscent of metastability.

On a higher time scale and on a higher cognitive level, it is easy to think of various phenomenons which - even if they are only loosely related to the rigorously characterized kind of metastability this thesis is concerned with-are evocative of the phenomenon of metastability. One of them for example is the "eureka effect", which refers to the common experience in which one suddenly come to understand a concept or a problem that was previously incomprehensible for a long time. There is a plethora of examples of such events in everyone's life as well as in the history of scientific discoveries, starting with a wellknown (but fictional) story about Archimedes (the french mathematician and polymath Henri Poincaré is also known to have reported such experiences in a famous conference entitled "L'invention mathématiques" see [POI08]). Such events, in which an individual goes from a state of cognitive confusion to a state of sudden and unexpected comprehension, 


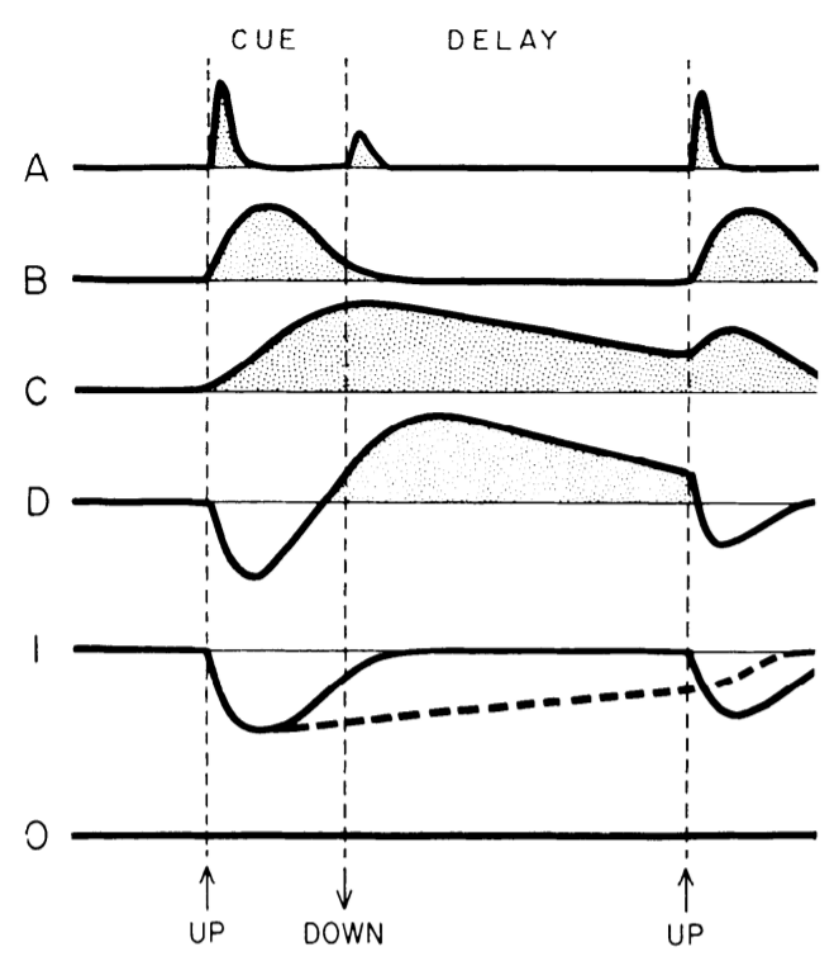

Figure 1.1: The figure showing the different type of neuronal responses in the original Fuster's article.

are reminiscent of a dynamical phase transition, i.e. of metastability, even if it is only on a metaphorical level. Other events of this type are for example the occurrence of some mental diseases, such as schizophrenia, which are known to be dormant for years, and to usually emerge suddenly after a psychotic breakdown either in a seemingly random fashion or triggered by a disruptive event such as the use of some drug, or a perturbation of the environment of the individual.

All this being said, it seems that we lack a formal and rigorous framework to address the subject, which is usually considered in neuroscience from an experimental and macroscopic perspective. The present work can be seen as an attempt to fill this gap. We propose ourselves to address the subject from the statistical physics point of view mentioned above, focusing on the asymptotic law of the time of extinction of finite versions of the system of interacting neurons defined in the previous section. As can be seen from simulations (see Figure 1.2 and Figure 1.3), for the version of the system in which the graph $G$ is the one dimensional lattice with nearest neighbors interaction (i.e. the instantiation studied in [FGGL18]), the system apparently presents a metastable behavior for small values of the parameter $\gamma$, while it seems to be highly non-metastable for high values of $\gamma$. The result of a simulation is nonetheless not a proof, and the purpose of this thesis is to study this model by giving mathematical proofs. This work is to the best of our knowledge the first one to address the problem of metastability in biological neural networks in a well-defined mathematical framework, by means of rigorous proofs. 


\section{Simulations for high values of $\gamma$}
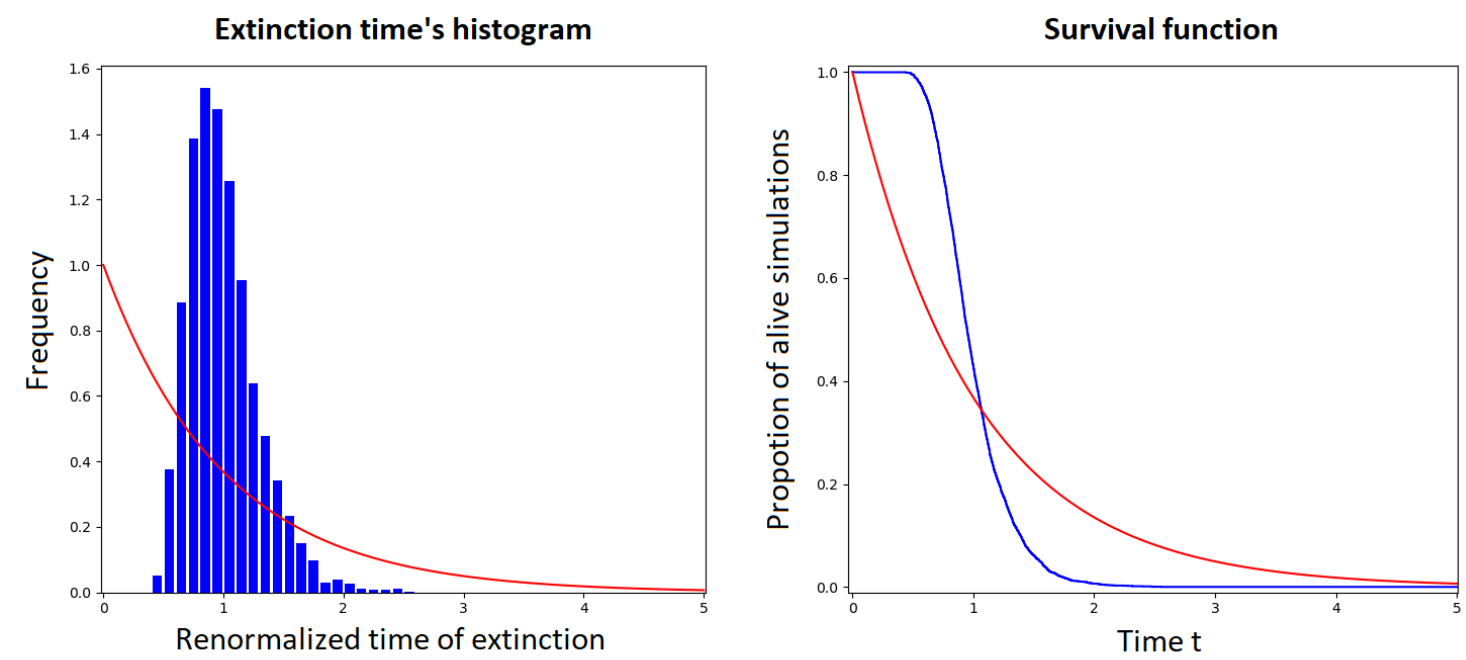

Trajectory

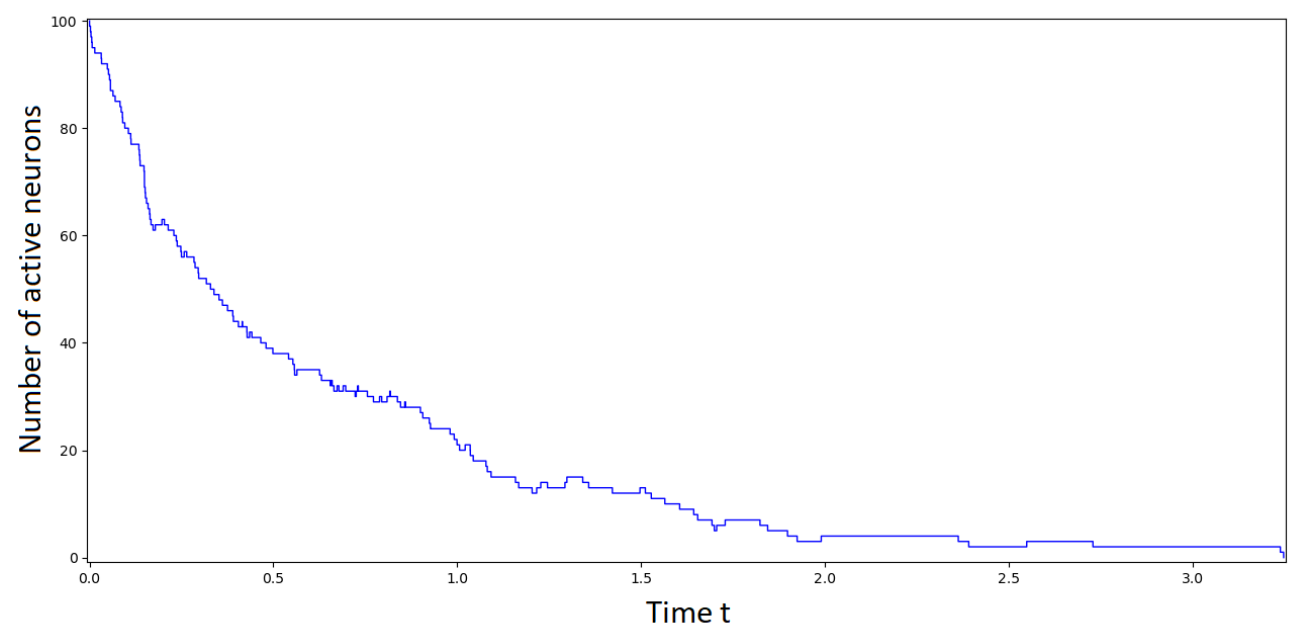

Figure 1.2: Simulation of the model with $\gamma=2$ for a system with 100 neurons. On the top left figure the histogram of the renormalized time of extinction is plotted. The histogram itself is normalized so that the area under the curve is equal to one. On the top right figure, the survival function is plotted (in blue). The red curve is the graph of $t \mapsto e^{-t}$ in both plot. Both are constructed on the same data set, which consists on 3000 simulation of the process. One can observe on the histogram and the survival function that the data are not exponentially distributed at all, instead the histogram seems to have its values concentrated around 1. On the bottom figure a typical trajectory of the process is plotted. One can observe that the number of active neurons constantly decreases and rapidly reaches 0 . No metastability can be seen in these figures. 


\section{Simulations for low values of $\gamma$}
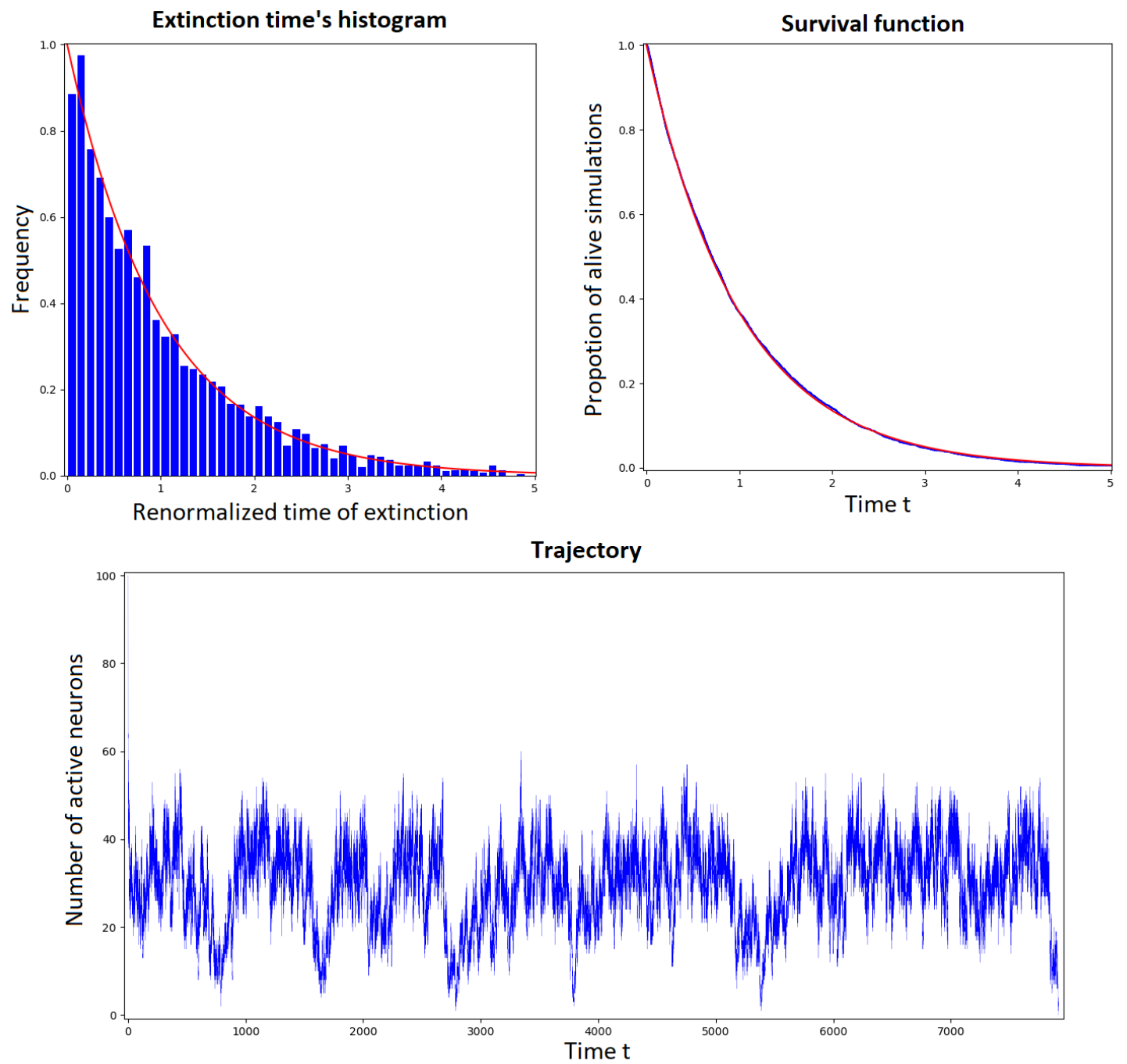

Figure 1.3: Simulation of the model with $\gamma=0.35$ for a system with 100 neurons. On the top left figure the histogram of the renormalized time of extinction is plotted. The histogram itself is normalized so that the area under the curve is equal to one. On the top right figure, the survival function is plotted (in blue). The red curve is the graph of $t \mapsto e^{-t}$ in both plot. Both are constructed on the same data set, which consists on 3000 simulations of the process. One can observe that the data are well approximated by an exponential distribution, as a matter of fact, the survival function is almost indistinguishable from the exponential function. On the bottom figure a typical trajectory of the process is plotted. One can observe that after a rapid decrease the number of active neurons stabilizes in pseudo-stationary state and oscillates before finally dying because of a sequence of abnormally close leaks. This behavior is typical of a metastable dynamic.

\subsection{Results presented in this thesis}

Most of the work of this thesis is concerned with the time of extinction of finite versions of the system defined above, as we consider the asymptotic distribution of these time of 
extinction to be the signature of the metastability or non-metastability of the systems considered, as explained in the previous section.

In Chapter 2, we present the result of phase transition proven in [FGGL18] in the onedimensional lattice setting, and we show that it can be generalized to the process defined on the lattice of any dimension. In Chapter 3 we study the process in the one-dimensional lattice setting of [FGGL18] and we prove that, when there is finitely many neurons, its time of extinction is asymptotically memory-less when $\gamma$ is taken sufficiently small. In order to build the proof of this result we obtain various intermediary results related to the invariant measure of the infinite process. The material of this chapter has been published in Journal of Statistical Physics. In Chapter 4 we show that, in the one-dimensional lattice setting, the result of metastability doesn't hold when $\gamma$ is taken big enough. In fact in this case, the time of extinction is asymptotically deterministic. In Chapter 5 we turn ourselves to the process defined on the complete graph, and prove that in this case the result of metastability holds for any $\gamma>0$. The material of these two last chapters has been accepted for publication in Stochastic Processes and their Applications. Finally we conclude in Chapter 6 and we draw possible future lines of research. 


\section{Chapter 2}

\section{Phase transition in the lattice}

\subsection{Phase transition in the one-dimensional lattice and gen- eralization to the d-dimensional lattice}

The first result obtained on our model is the result of phase transition that was proven in [FGGL18]. In this last paper, the process considered is the process defined on the infinite one-dimensional lattice, that is the process defined as in Section 1.2 of the introduction when we assume the following.

Assumption 2.1.1 Let $S=\mathbb{Z}$ and for all $i \in S, \mathbb{V}_{i}=\{i-1, i+1\}$.

In this process, the neurons are indexed on the integers and each neuron has only two presynaptic neurons, which are his immediate neighbors on the left and on the right. Notice that while the graph of interaction was thought as a directed graph in the definition of Section 1.2, in this specific case if $j \in \mathbb{V}_{i}$ then $i \in \mathbb{V}_{j}$ as well, so that the graph is actually undirected. This will be the case for all the graphs studied in this thesis.

It was proven in [FGGL18] that this instantiation of the process is subject to a phase transition with respect to the leakage parameter $\gamma$. More precisely, the following theorem was proven.

Theorem 2.1.2 Under Assumption 2.1.1 and assuming that $X_{i}(0) \geq 1$ for all $i \in S$, there exists a critical value $\gamma_{c}$ for the parameter $\gamma$, with $0<\gamma_{c}<\infty$, such that for any $i \in S$

$$
\mathbb{P}\left(N _ { i } \left([0, \infty[)<\infty)=1 \text { if } \gamma>\gamma_{c}\right.\right.
$$

and

$$
\mathbb{P}\left(N _ { i } \left([0, \infty[)=\infty)>0 \text { if } \gamma<\gamma_{c}\right.\right.
$$

In words there exists a critical value $\gamma_{c}$ that is such that each neuron in the system continues spiking infinitely often with positive probability when $\gamma$ is below it, or stops 
spiking once for all after some time when $\gamma$ is above it. The last sentence can be rephrased saying that for $\gamma>\gamma_{c}$ all neurons become quiescent almost surely as the time goes to infinity while for $\gamma<\gamma_{c}$ every neuron stays infinitely often active with positive probability.

In this chapter we show that the result of phase transition in the one dimensional lattice process can be generalized to the multi-dimensional process. Let $d \geq 1$ be some integer. In the following we consider the process defined on the infinite d-dimensional lattice, that is the process defined as in Section 1.2 of the introduction when we assume the following.

Assumption 2.1.3 $S=\mathbb{Z}^{d}$ and for all $i \in S, \mathbb{V}_{i}=\left\{j \in \mathbb{Z}^{d}:\|i-j\|_{d}=1\right\}$, where $\|\cdot\|_{d}$ is the $d$-dimensional norm defined for any $i \in \mathbb{Z}^{d}$ by

$$
\|i\|_{d} \stackrel{\text { def }}{=} \sum_{k=1}^{d}\left|i_{k}\right| .
$$

Notice that if $d=1$ Assumption 2.1.3 above is simply Assumption 2.1.1. For any $i \in \mathbb{Z}$ and $t \geq 0$ we write $\eta_{i}(t)=\mathbb{1}_{X_{i}(t)>0}$, and $\eta(t)=\left(\eta_{j}(t)\right)_{j \in S}$. The process $(\eta(t))_{t \geq 0}$ is a Markov process taking value in $\{0,1\}^{\mathbb{Z}^{d}}$. The generator of this process is given by

$$
\mathscr{L}^{d} f(\eta)=\gamma \sum_{i \in \mathbb{Z}^{d}}\left(f\left(\pi_{i}^{\dagger, d}(\eta)\right)-f(\eta)\right)+\sum_{i \in \mathbb{Z}^{d}} \eta_{i}\left(f\left(\pi_{i}^{d}(\eta)\right)-f(\eta)\right),
$$

where the $\pi_{i}^{\dagger, d}$ 's and $\pi_{i}^{d}$ 's are maps from $\{0,1\}^{\mathbb{Z}^{d}}$ to $\{0,1\}^{\mathbb{Z}^{d}}$ defined for any $i \in \mathbb{Z}^{d}$ as follows:

$$
\left(\pi_{i}^{\dagger, d}(\eta)\right)_{j}= \begin{cases}0 & \text { if } j=i \\ \eta_{j} & \text { otherwise }\end{cases}
$$

and

$$
\left(\pi_{i}^{d}(\eta)\right)_{j}= \begin{cases}0 & \text { if } j=i \\ \max \left(\eta_{i}, \eta_{j}\right) & \text { if }\|i-j\|_{d}=1 \\ \eta_{j} & \text { otherwise. }\end{cases}
$$

For any $\eta \in\{0,1\}^{\mathbb{Z}^{d}}$ we will write $\left(\eta^{\eta}(t)_{t \geq 0}\right)$ for the process having generator (2.1) and initial state $\eta$. By convention when no indication is given the initial state of the process is the state where all neurons are actives.

The existence and uniqueness of this process are guaranteed by standard theory in interacting particle systems (see Theorem A.0.5 in appendix, or Theorem 3.9 in [LIG85]). This is essentially a consequence of the fact that the range of the interaction is finite as well as the translation invariance of the process (the local dynamic doesn't depend on the neuron we are looking at).

We are aimed to prove the following result

Theorem 2.1.4 Under Assumption 2.1.3 and assuming that $X_{i}(0) \geq 1$ for all $i \in \mathbb{Z}^{d}$, 
there exists a critical value $\gamma_{c}^{(d)}$ for the parameter $\gamma$, with $0<\gamma_{c}^{(d)}<\infty$, such that for any $i \in \mathbb{Z}^{d}$

$$
\mathbb{P}\left(N _ { i } \left([0, \infty[)<\infty)=1 \text { if } \gamma>\gamma_{c}^{(d)}\right.\right.
$$

and

$$
\mathbb{P}\left(N _ { i } \left([0, \infty[)=\infty)>0 \text { if } \gamma<\gamma_{c}^{(d)}\right.\right.
$$

\subsection{The d-dimensional dual process}

An important tool that we are going to use in order to reach our purpose is the theory of duality. The existence of a dual process was already crucial in the proof of phase transition for the one-dimensional setting studied in [FGGL18], and it will not only be useful here to generalize the phase transition result to the dimension $d$ but also to prove the result of metastability of the next chapter. The theory of duality thus play an important role in half of this thesis, and a brief reminder of its main results is given in Appendix A. A more detailed introduction to the subject can be found in [BG77].

As an application of Theorem A.0.5 Our process $\left(\eta_{t}\right)_{t \geq 0}$ admits a dual. This is a consequence of the important fact that the process is additive in the sense that for any $i \in \mathbb{Z}^{d}$ and $A \in \mathscr{P}\left(\mathbb{Z}^{d}\right)$ (here $\mathscr{P}\left(\mathbb{Z}^{d}\right)$ is only an alternative way to represent the state space ${ }^{1}$ ) the following holds

$$
\pi_{i}^{\dagger, d}(A)=\bigcup_{j \in A} \pi_{i}^{\dagger, d}(\{j\})
$$

and

$$
\pi_{i}^{d}(A)=\bigcup_{j \in A} \pi_{i}^{d}(\{j\})
$$

The dual process is denoted $\left(\tilde{\eta}_{t}\right)_{t \geq 0}$. It is the process taking value in $\{0,1\}^{\mathbb{Z}^{d}}$ with the following infinitesimal generator

$$
\tilde{\mathscr{L}}^{d} g(F)=\gamma \sum_{i \in \mathbb{Z}}\left(g\left(\tilde{\pi}_{i}^{\dagger, d}(F)\right)-g(F)\right)+\sum_{i \in \mathbb{Z}} \xi_{i}\left(g\left(\tilde{\pi}_{i}^{d}(F)\right)-g(F)\right)
$$

where $g$ is a cylindrical function and $F \in \mathscr{P}\left(\mathbb{Z}^{d}\right)$. The $\tilde{\pi}_{i}^{\dagger, d}$ 's and $\tilde{\pi}_{i}^{d}$ 's are defined as follows:

\footnotetext{
${ }^{1}$ Indeed we can identify the state space $\{0,1\}^{\mathbb{Z}^{d}}$ to the state space $\mathscr{P}\left(\mathbb{Z}^{d}\right)$, the set of all subsets of $\mathbb{Z}^{d}$, as any element $\eta$ of $\{0,1\}^{\mathbb{Z}^{d}}$ can be bijectively mapped to an element $A$ of $\mathscr{P}\left(\mathbb{Z}^{d}\right)$-via the obvious relation $A=\left\{i \in \mathbb{Z}\right.$ such that $\left.\eta_{i}=1\right\}$ - so that we can indifferently use both ways. In practice we will use both of them without explicitly specifying it each time. By convention we will generally use letters such as $\eta, \xi$ and $\zeta$ for elements of $\{0,1\}^{\mathbb{Z}^{d}}$ and letters such as $A, B$ and $C$ for elements of $\mathscr{P}\left(\mathbb{Z}^{d}\right)$. What we mean should be clear from the context.
} 


$$
\tilde{\pi}_{i}^{\dagger, d}(F)=F \backslash\{i\}
$$

for all $F \in \mathscr{P}\left(\mathbb{Z}^{d}\right)$ and $i \in \mathbb{Z}^{d}$, and

$$
\tilde{\pi}_{i}^{d}(\{j\})= \begin{cases}\emptyset & \text { if } j=i \\ \{i, j\} & \text { if }\|i-j\|_{d}=1 \\ \{j\} & \text { otherwise }\end{cases}
$$

for all $i \in \mathbb{Z}^{d}$ and $j \in \mathbb{Z}^{d}$, the map for bigger sets $F \in \mathscr{P}\left(\mathbb{Z}^{d}\right)$ being given for all $i \in \mathbb{Z}^{d}$ by

$$
\tilde{\pi}_{i}^{d}(F)=\bigcup_{j \in F} \tilde{\pi}_{i}^{d}(\{j\})
$$

We adopt the same convention regarding the notation of the initial state of the process as for the original process. The important result that links the original process with its dual is the following

Theorem 2.2.1 For any $A, B \in \mathscr{P}\left(\mathbb{Z}^{d}\right)$ such that at least one of them is finite, and for any $t \geq 0$ we have

$$
\mathbb{P}\left(\eta^{A}(t) \cap B \neq \emptyset\right)=\mathbb{P}\left(\tilde{\eta}^{B}(t) \cap A \neq \emptyset\right) .
$$

Intuitively this last theorem means that the dual process essentially behave like the initial process with the time reversed.

Let $\mathbf{0}_{d}=(0, \ldots 0) \in \mathbb{Z}^{d}$ denotes the origin of the lattice $\mathbb{Z}^{d}$. We define the time of extinction of this dual process

$$
\tilde{\kappa}^{\mathbf{0}_{d}}=\inf \left\{t \geq 0: \tilde{\eta}^{\mathbf{0}_{d}}(t)=\emptyset\right\} .^{2}
$$

We have the following proposition, which we will use in the proof of the following section.

Proposition 2.2.2 If $\gamma>2 d+1$, then $\mathbb{P}\left(\tilde{\kappa}^{\mathbf{0}_{d}}=\infty\right)=0$.

Proof: This is proven by a coupling of $\left(\left|\tilde{\eta}^{\mathbf{0}_{d}}(t)\right|\right)_{t \geq 0}$ with a branching process, that is to say the joint construction of both processes on the same probability space.

First we construct an intermediate process $\left(Y_{t}\right)_{t \geq 0}$. The coupling is done as follows. At time $0, Y_{0}=1$. For any $t \geq 0$ we define the set $\mathscr{N}(t)$ of neighbors of $\tilde{\eta}^{\mathbf{0}_{d}}(t)$, including $\tilde{\eta}^{\mathbf{0}_{d}}(t)$ itself, that is

$$
\mathscr{N}(t) \stackrel{\text { def }}{=}\left\{j \in \mathbb{Z}^{d}:\|j-i\|_{d} \leq 1 \text { for some } i \in \tilde{\eta}^{\mathbf{0}_{d}}(t)\right\} .
$$

\footnotetext{
${ }^{2}$ Here and in the following, as an abuse of notation, the initial configuration is denoted $\mathbf{0}_{d}$ while strictly speaking we should write $\left\{\mathbf{0}_{d}\right\}$. This kind of abuse of notation will be used repeatedly throughout this thesis without further remark.
} 
Whenever a transformation $\tilde{\pi}_{i}^{\dagger, d}$ occurs with $i \in \tilde{\eta}^{\mathbf{0}_{d}}(t), Y_{t}$ decreases by one. If $Y_{t}>$ $\left|\tilde{\eta}^{\mathbf{d}_{d}}(t)\right|$ for some $t \geq 0$ then additionally $\left(Y_{t}\right)_{t \geq 0}$ also decreases by one at rate $Y_{t}-\left|\tilde{\eta}^{\mathbf{0}_{d}}(t)\right|$. Whenever a transformation $\tilde{\pi}_{i}^{d}$ occurs with $i \in \mathscr{N}(t), Y_{t}$ increases by one. When such a transformation occurs, $\left|\tilde{\eta}^{\mathbf{0}_{d}}(t)\right|$ can either increase by one, stay the same, of decrease by one, depending on the configuration of the process at that time, so that with this coupling we obviously have

$$
\left|\tilde{\eta}^{\mathbf{0}_{d}}(t)\right| \leq Y_{t} \text { for any } t \geq 0
$$

Moreover the increase rate of $Y_{t}$ at any time $t \geq 0$ is equal to $|\mathscr{N}(t)|$, which is maximal when the configuration of $\tilde{\eta}^{\mathbf{0}_{d}}(t)$ is such that every element is isolated ; when this is the case, conditioned on $\left|\tilde{\eta}^{\mathbf{0}_{d}}(t)\right|=n$, we have $|\mathscr{N}(t)|=n(2 d+1)$. Therefore, by the inequality above the increase rate of $Y_{t}$ is always less than $Y_{t}(2 d+1)$, so that it is easy to see that $\left(Y_{t}\right)_{t \geq 0}$ can be coupled with a continuous time branching process $\left(Z_{t}\right)_{t \geq 0}$ having birth rate $2 d+1$ and death rate $\gamma$ (see Section B.3 in the Appendix for details) in such a way that

$$
Y_{t} \leq Z_{t} \text { for any } t \geq 0 \text {. }
$$

Moreover, by Proposition B.3.1 in Appendix B, we have

$$
\mathbb{E}\left(Z_{t}\right)=e^{-(\gamma-(2 d+1)) t}
$$

Therefore, by Markov inequality

$$
\mathbb{P}\left(\tilde{\eta}^{\mathbf{0}_{d}}(t) \neq \emptyset\right) \leq \mathbb{P}\left(Z_{t} \geq 1\right) \leq e^{-(\gamma-(2 d+1)) t}
$$

and when $\gamma>2 d+1$ the right-hand side converges to 0 when $t$ goes to $\infty$, which ends the proof.

\subsection{Proof of Theorem 2.1.4}

If $d=1$, then Theorem 2.1.4 is Theorem 2.1.2, which has already been proven in [FGGL18], so that we can assume $d \geq 2$.

Moreover it is sufficient to consider the case $i=\mathbf{0}_{d}$. Notice that $\mathbb{P}\left(N_{\mathbf{0}_{d}}([0, \infty[)=\infty)\right.$ is a non-increasing function of $\gamma$ (if not obvious, this results from similar reasoning as the proof of Lemma 5 in [FGGL18]), so that it suffices to find two values of $\gamma$ such that the probability is equal to 0 for the first value and strictly bigger than 0 for the second value.

We consider the sub-critical case first. The result follows directly from the one-dimensional phase transition result. Indeed $\mathbb{Z}^{d}$ contains a copy of $\mathbb{Z}$, so that by an obvious coupling we have that

$$
\mathbb{P}\left(N _ { \mathbf { 0 } _ { d } } \left([0, \infty[)=\infty) \geq \mathbb{P}\left(N_{0}([0, \infty[)=\infty),\right.\right.\right.
$$

where in the right-hand side $N_{0}([0, \infty[)$ denotes the number of spikes of neuron 0 for the one-dimensional process (that is the process under Assumption 2.1.1) while in the left-hand 
side $N_{\mathbf{0}_{d}}\left(\left[0, \infty[)\right.\right.$ denotes the number of spikes of neuron $\mathbf{0}_{d}$ for the d-dimensional process (that is the process under assumption 2.1.3). It follows that for any $\gamma<\gamma_{c}$ (where $\gamma_{c}$ is the critical value for the one-dimensional process), we have

$$
\mathbb{P}\left(N_{\mathbf{0}_{d}}([0, \infty[)=\infty)>0,\right.
$$

which ends the proof for the sub-critical part.

For the super-critical part we have by duality that

$$
\mathbb{P}\left(\eta_{\mathbf{0}_{d}}(t)=1\right)=\mathbb{P}\left(\tilde{\kappa}^{\mathbf{0}_{d}}>t\right) \underset{t \rightarrow \infty}{\longrightarrow} \mathbb{P}\left(\widetilde{\kappa}^{\mathbf{0}_{d}}=\infty\right),
$$

and the limit is equal to 0 if we choose $\gamma>2 d+1$ by Proposition 2.2.2. If $\mathbb{P}\left(N_{\mathbf{0}_{d}}([0, \infty[)=\right.$ $\infty)$ were strictly positive then the limit above could not be 0 , so that the proof is over. 


\section{Chapter 3}

\section{Metastability in the sub-critical region for the one-dimensional lattice process}

\subsection{Definitions and result}

In this chapter we study exclusively the one-dimensional lattice process, that is the process obtained under Assumption 2.1.1. As stated in the previous chapter, it was proven in [FGGL18] that this process is subject to a phase transition and possesses a critical value that we denote $\gamma_{c}$.

As usual, the key process we are going to work with is the spiking rates process. For any $i \in \mathbb{Z}$ and $t \geq 0$ we write $\xi_{i}(t)=\mathbb{1}_{X_{i}(t)>0}$, and $\xi(t)=\left(\xi_{j}(t)\right)_{j \in S}$. The main object we are interested in is the resulting process $(\xi(t))_{t \geq 0}$. It is the interacting particle system which dynamic is given by the generator in (1.4), when you replace $S$ by $\mathbb{Z}$ and $\mathbb{V}_{i}$ by $\{i-1, i+1\}$, that is

$$
\mathscr{L}^{1} f(\eta)=\gamma \sum_{i \in \mathbb{Z}}\left(f\left(\pi_{i}^{\dagger, 1}(\xi)\right)-f(\xi)\right)+\sum_{i \in \mathbb{Z}} \eta_{i}\left(f\left(\pi_{i}^{1}(\xi)\right)-f(\xi)\right),
$$

where the $\pi_{i}^{\dagger, 1}$ 's and $\pi_{i}^{1}$ 's are maps from $\{0,1\}^{\mathbb{Z}}$ to $\{0,1\}^{\mathbb{Z}}$ defined for any $i \in \mathbb{Z}$ as follows:

$$
\left(\pi_{i}^{\dagger, 1}(\xi)\right)_{j}= \begin{cases}0 & \text { if } j=i \\ \eta_{j} & \text { otherwise }\end{cases}
$$

and

$$
\left(\pi_{i}^{1}(\xi)\right)_{j}= \begin{cases}0 & \text { if } j=i \\ \max \left(\eta_{i}, \eta_{j}\right) & \text { if } j \in\{i-1, i+1\} \\ \eta_{j} & \text { otherwise. }\end{cases}
$$


We adopt the same convention as in the previous chapter regarding the notation for the initial state. Moreover, for any $\xi \in\{0,1\}^{\mathbb{Z}}$, we define the extinction time of the process $\left(\xi^{\xi}(t)\right)_{t \geq 0}$

$$
\tau^{\xi}=\inf \left\{t \geq 0: \xi^{\xi}(t)_{i}=0 \text { for any } i \in \mathbb{Z}\right\},
$$

with the convention that $\inf \emptyset=+\infty$.

We also consider a finite version of the process $(\xi(t))_{t>0}$, where the neurons aren't anymore in the infinite lattice but only in a finite portion of it. Fix some integer $N \geq 0$ and let $S_{N}=\llbracket-N, N \rrbracket$ (where $\llbracket-N, N \rrbracket$ is a short-hand for $\mathbb{Z} \cap[-N, N]$ ). For any $i \in S_{N}$ let the set $\mathbb{V}_{N, i}$ be defined by

$$
\mathbb{V}_{N, i}= \begin{cases}\{i-1, i+1\} & \text { if } i \in \llbracket-(N-1), N-1 \rrbracket \\ \{N-1\} & \text { if } i=N \\ \{-(N-1)\} & \text { if } i=-N\end{cases}
$$

We write $\left(\xi_{N}(t)\right)_{t>0}$ for the finite version of the lattice process, that is to say the process taking value in $\{0,1\}^{2 N+1}$ which dynamic is given by the generator in (1.4) when you replace $S$ by $S_{N}$ and $\mathbb{V}_{i}$ by $\mathbb{V}_{N, i}$ for any $i \in S_{N}$, that is

$$
\mathscr{L}_{N}^{1} f(\xi)=\gamma \sum_{-N \leq i \leq N}\left(f\left(\pi_{N, i}^{\dagger, 1}(\xi)\right)-f(\xi)\right)+\sum_{-N \leq i \leq N} \eta_{i}\left(f\left(\pi_{N, i}^{1}(\xi)\right)-f(\xi)\right),
$$

where the $\pi_{N, i}^{\dagger, 1}$ 's and $\pi_{N, i}^{1}$ 's are maps from $\{0,1\}^{2 N+1}$ to $\{0,1\}^{2 N+1}$, which definition agree with (3.2) and (3.3) respectively for any $i \in \llbracket-N, N \rrbracket$.

The existence and uniqueness of both the infinite and the finite processes are guaranteed by standard theory in interacting particle systems (see previous chapter).

Let $\tau_{N}$ denote the time of extinction of this finite process, that is to say the first time at which all neurons are quiescent. Formally

$$
\tau_{N} \stackrel{\text { def }}{=} \inf \left\{t \geq 0: \xi_{N}(t)_{i}=0 \text { for any } i \in \llbracket-N, N \rrbracket\right\} .
$$

The purpose of this chapter is to prove one of our main theorems, which is the following.

Theorem 3.1.1 There exists $\gamma_{c}^{\prime}$ such that if $0<\gamma<\gamma_{c}^{\prime}$, then we have the following convergence

$$
\frac{\tau_{N}}{\mathbb{E}\left(\tau_{N}\right)} \underset{N \rightarrow \infty}{\stackrel{\mathscr{D}}{\longrightarrow}} \mathscr{E}(1)
$$

For this purpose we will need an extensive study of the infinite process, and specifically of its asymptotic behavior. 


\subsection{The graphical construction}

Inspired by the graphical construction introduced by Harris in [HAR78] we consider an alternative definition of our process. The reason for introducing this alternative construction is that it proved itself to be a powerful tool in the field of interacting particle systems. It permits to construct the infinite process, the finite process, as well as the semi-infinite processes (see next section) on the same probability space, which allows us to use extensively the technique of coupling - which we already used in the previous chapter - i.e. to construct the processes in such a way that they satisfy a given relation for every realization (every point on the probability space), allowing us to obtain inequalities about the probability of some events involving them (see [THO95] for a review on coupling).

For any $i \in \mathbb{Z}$ let $\left(N_{i}^{\dagger}(t)\right)_{t \geq 0}$ be the homogeneous Poisson process of rate $\gamma$ mentioned in the introduction, and let $\left(N_{i}^{*}(t)\right)_{t \geq 0}$ be an homogeneous Poisson process of rate 1 . Let $\left(T_{i, n}^{\dagger}\right)_{n \geq 0}$ and $\left(T_{i, n}^{*}\right)_{n \geq 0}$ be their respective jump times. We also impose that the Poisson processes are defined on the same probability space $(\Omega, \mathscr{F}, \mathbb{P})$ and are mutually independent.

Moreover we consider the time-space diagram $\mathbb{Z} \times \mathbb{R}_{+}$, and for any realization of the Poisson processes, we do the following:

- for all $i \in \mathbb{Z}$ and $n \in \mathbb{N}$ put a " $\delta$ " mark at the point $\left(i, T_{i, n}^{\dagger}\right)$,

- for all $i \in \mathbb{Z}$ and $n \in \mathbb{N}$ put an arrow pointing from $\left(i, T_{i, n}^{*}\right)$ to $\left(i+1, T_{i, n}^{*}\right)$ and another pointing from $\left(i, T_{i, n}^{*}\right)$ to $\left(i-1, T_{i, n}^{*}\right)$.

That way we obtain a random graph $\mathscr{G}$ which consists of the time-space diagram $\mathbb{Z} \times \mathbb{R}$ augmented by the set of " $\delta$ " marks and horizontal arrows we just described, and which is constructed on the underlying probability space $(\Omega, \mathscr{F}, \mathbb{P})$.

We call a time segment any subset of $\mathbb{Z} \times \mathbb{R}$ of the form $\left\{(i, s), t \leq s \leq t^{\prime}\right\}$, for some $i \in \mathbb{Z}$ and some $t<t^{\prime}$. Moreover, for some $i, j \in \mathbb{Z}$ and $t<t^{\prime}$, and for any realization of the graph $\mathscr{G}$, we say that there is a path from $(i, t)$ to $\left(j, t^{\prime}\right)$ in $\mathscr{G}$ if there is a connected chain of time segment and arrows leading from $(i, t)$ to $\left(j, t^{\prime}\right)$. We say that it is a valid path if it satisfies the following constraints:

- it never cross a " $\delta$ " mark,

- when moving upward, we never cross the rear side of an arrow.

We write $(i, t) \longrightarrow\left(j, t^{\prime}\right)$ when there is a valid path from $(i, t)$ to $\left(j, t^{\prime}\right)$ in $\mathscr{G}$. With this construction we can easily give the following characterization of our stochastic process. For any $A \in \mathscr{P}(\mathbb{Z})$, and for any $t \geq 0$ :

$$
\xi^{A}(t)=\{j \in \mathbb{Z}:(i, 0) \longrightarrow(j, t) \text { for some } i \in A\} .^{1}
$$

As a reward for our hard work we immediately obtain the two following results.

\footnotetext{
${ }^{1}$ As usual we are identifying the state space $\{0,1\}^{\mathbb{Z}}$ with the state space $\mathscr{P}(\mathbb{Z})$. This convention will now be used throughout this thesis without further remark.
} 
Proposition 3.2.1 Let $A \subset B \subset \mathbb{Z}$, then for any $t \geq 0$ we have

$$
\xi^{A}(t) \subset \xi^{B}(t)
$$

Moreover, the process is additive in the following sense

$$
\xi^{A}(t)=\bigcup_{i \in A} \xi^{i}(t)
$$

Proof: It follows from the graphical construction. For the first part of the proposition it suffices to notice that if $(i, 0) \longrightarrow(j, t)$ for some $i$ in $A$ and some $t \geq 0$ then $(j, t) \in \xi^{B}(t)$ as $i$ belongs to $B$ as-well. The second part is simply a reformulation of the definition.

Remark 3.2.2 Proposition 3.2.1 remains obviously true if you replace $(\xi(t))_{t \geq 0}$ by the finite process $\left(\xi_{N}(t)\right)_{t \geq 0}$ or one of the semi-infinite processes which we introduce in the next section. Moreover, the second part of the proposition is also a consequence of Theorem A.0.5 in the Appendix together with the additivity of the maps defining the generator of the process, but we included the result here to give a taste of the simplicity and practicality of the graphical construction.

\subsection{The semi-infinite processes}

In order to prove the result of metastability in which we are interested we need to introduce restricted versions of the infinite system of spiking neurons.

For any $N \in \mathbb{Z}$, the right semi-infinite process, which we denote $\left(\xi_{[N,+\infty[}(t)\right)_{t \geq 0}$, is the process taking values in $\mathscr{P}(\llbracket N,+\infty \llbracket)$ corresponding to the process we obtain from (1.4) when the set of neurons is given by $S_{[N, \infty[}=\llbracket N,+\infty \llbracket$ and when the set of presynaptic neurons for any neuron $i \in S_{[N, \infty[\text {, is defined as }}$

$$
\mathbb{V}_{i}^{[N, \infty[}= \begin{cases}\{i-1, i+1\} & \text { if } i \neq N, \\ \{N+1\} & \text { otherwise }\end{cases}
$$

In term of the graphical construction $\left(\xi_{[N,+\infty[}(t)\right)_{t \geq 0}$ is the process constructed as $(\xi(t))_{t \geq 0}$, with the random graph $\mathscr{G}$, but using only the $\delta$ 's and arrows from the sub diagram $\llbracket N,+\infty \llbracket \times \mathbb{R}_{+}$. The left semi-infinite process $\left(\xi_{-\infty, N]}(t)\right)_{t \geq 0}$ is defined in a similar way, on $\mathscr{P}(\rrbracket-\infty, N \rrbracket)$, considering only $\delta$ 's and arrows on the sub diagram $\rrbracket-\infty, N \rrbracket \times \mathbb{R}_{+}$. We adopt the usual convention regarding the notation of the initial state. Notice that all these processes are constructed on the same probability space $(\Omega, \mathscr{F}, \mathbb{P})$, and that we have the following monotonicity relationships:

$$
\forall A \in \mathscr{P}(\llbracket N,+\infty \llbracket), \forall t \geq 0, \xi_{[N,+\infty[}^{A}(t) \subset \xi^{A}(t),
$$




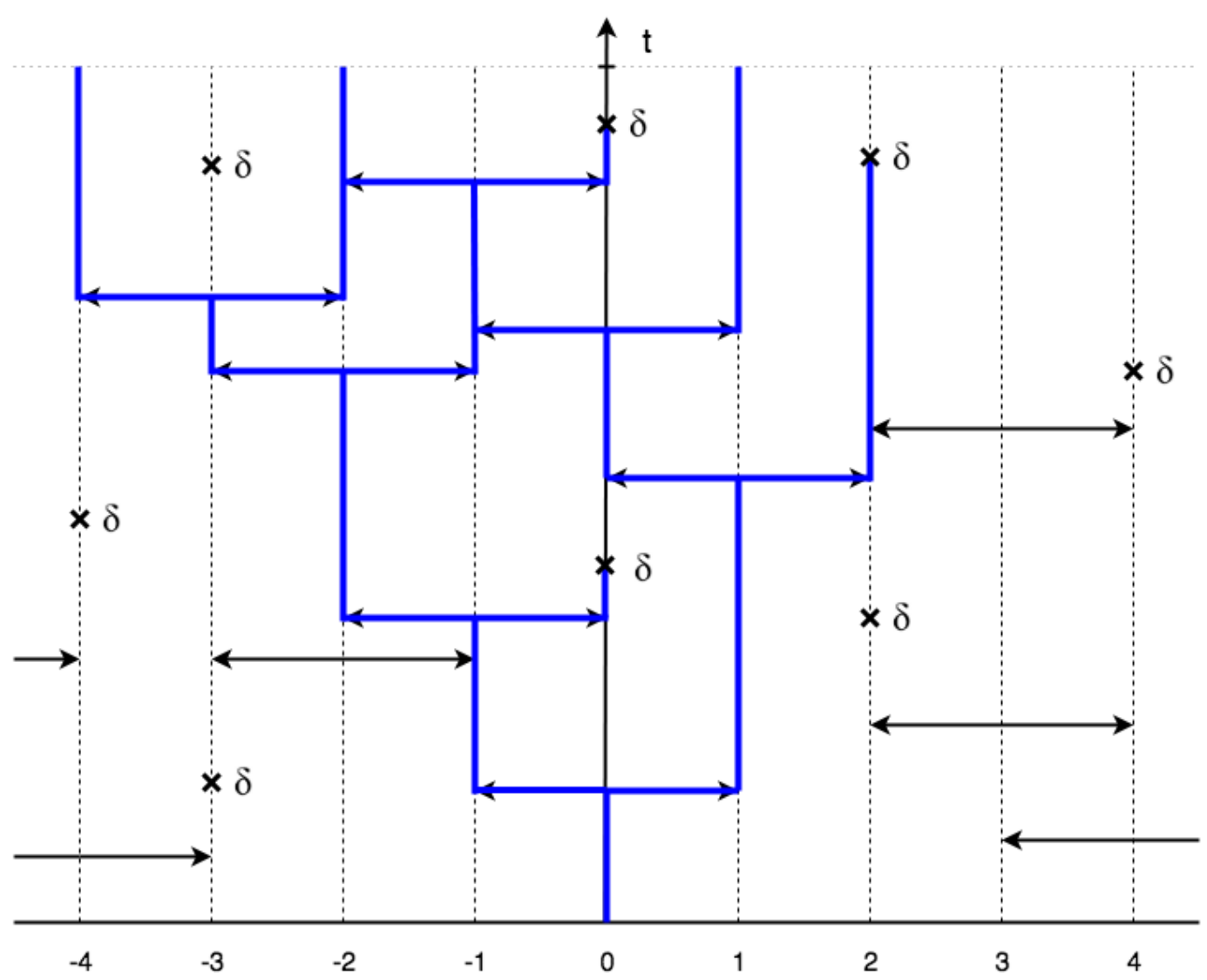

Figure 3.1: A realization of the random graph $\mathscr{G}$. In blue all the possible valid paths starting from $(0,0)$. Here the configuration of the process at time $t$ when the initial configuration is the singleton $\{0\}$ is the set $\{-4,-2,1\}$.

$$
\begin{gathered}
\forall A \in \mathscr{P}(\rrbracket-\infty, N \rrbracket), \forall t \geq 0, \xi_{]-\infty, N]}^{A}(t) \subset \xi^{A}(t), \\
\forall A \in \mathscr{P}(\llbracket-N, N \rrbracket), \quad \forall t \geq 0, \xi_{N}^{A}(t) \subset \xi^{A}(t) .
\end{gathered}
$$

The same way we defined an extinction time for the finite process, for any $N \in \mathbb{Z}$ and any initial configuration $A \in \mathscr{P}(\llbracket N,+\infty \llbracket)$ (resp. $A \in \mathscr{P}(\rrbracket-\infty, N \rrbracket)$ ) we define the extinction time of the right semi-infinite process (resp. left semi-infinite process) denoted $\tau_{[N,+\infty[}^{A}\left(\right.$ resp. $\left.\tau_{]-\infty, N]}^{A}\right)$. 


\subsection{The dual process}

\subsubsection{Formal definition of the dual process}

As explained in the previous chapter, the fact that our process is additive has the nice consequence that it has a dual again we refer to Appendix A for a quick reminder, or to [HAR76] and [BG77] for an in-depth presentation).

The dual process of our system of spiking neurons is defined on the state space $\mathscr{P}(\mathbb{Z})$ of finite subset of $\mathbb{Z}$ and has the following generator (see [FGGL18]):

$$
\tilde{\mathscr{L}}^{1} g(F)=\gamma \sum_{i \in \mathbb{Z}}\left(g\left(\tilde{\pi}_{i}^{\dagger, 1}(F)\right)-g(F)\right)+\sum_{i \in \mathbb{Z}} \xi_{i}\left(g\left(\tilde{\pi}_{i}^{1}(F)\right)-g(F)\right),
$$

where $g$ is a cylindrical function and $F \in \mathscr{P}(\mathbb{Z})$. The $\tilde{\pi}_{i}^{\dagger, 1}$ 's and $\tilde{\pi}_{i}^{1}$ 's are called the dual maps and are defined as follows:

$$
\tilde{\pi}_{i}^{\dagger, 1}(F)=F \backslash\{i\}
$$

for all $F \in \mathscr{P}(\mathbb{Z})$ and $i \in \mathbb{Z}$, and

$$
\tilde{\pi}_{i}^{1}(\{j\})= \begin{cases}\emptyset & \text { if } j=i \\ \{i, j\} & \text { if } j \in\{i-1, i+1\} \\ \{j\} & \text { otherwise }\end{cases}
$$

for all $i \in \mathbb{Z}$ and $j \in \mathbb{Z}$, the map for bigger sets $F \in \mathscr{P}(\mathbb{Z})$ being given for all $i \in \mathbb{Z}$ by

$$
\tilde{\pi}_{i}^{1}(F)=\bigcup_{j \in F} \tilde{\pi}_{i}^{1}(\{j\})
$$

We write $(\widetilde{\xi}(t))_{t \geq 0}$ for the process with generator (3.5). As well as for the original process we define, for any $A \in \mathscr{P}(\mathbb{Z})$, the extinction time for the dual-process:

$$
\tilde{\tau}^{A}=\inf \left\{t \geq 0: \widetilde{\xi}^{A}(t)=\emptyset\right\}
$$

The crucial point is that the original process and its dual are linked by the duality property, already given in the previous chapter (Theorem 2.2.1). This property will be crucial in the following sections of this chapter, where it will often be used in the following form:

$$
\mathbb{P}(\xi(t) \cap\{0\} \neq \emptyset)=\mathbb{P}\left(\widetilde{\xi}^{0}(t) \neq \emptyset\right)=\mathbb{P}\left(\tilde{\tau}^{0}>t\right) .
$$




\subsubsection{Graphical construction of the dual process}

It is also possible to give a graphical construction for the dual process. Again, for any $i \in \mathbb{N}$ and $n \in \mathbb{N}$, let's consider two independent homogeneous Poisson processes $\left(\tilde{N}_{i}^{*}(t)\right)_{t \geq 0}$ and $\left(\tilde{N}_{i}^{\dagger}(t)\right)_{t \geq 0}$ with intensity 1 and $\gamma$ respectively, and let $\left(\tilde{T}_{i, n}^{*}\right)_{n \geq 0}$ and $\left(\tilde{T}_{i, n}^{\dagger}\right)_{n \geq 0}$ be their respective jump times. As previously all the Poisson processes are assumed to be mutually independent.

We consider the time-space diagram $\mathbb{Z} \times \mathbb{R}_{+}$, and for any realization of the Poisson processes, we do the following:

- for all $i \in \mathbb{Z}$ and $n \in \mathbb{N}$ put a " $\delta$ " mark at the point $\left(i, \tilde{T}_{i, n}^{\dagger}\right)$,

- for all $i \in \mathbb{Z}$ and $n \in \mathbb{N}$ put an arrow pointing from $\left(i+1, \tilde{T}_{i, n}^{*}\right)$ to $\left(i, \tilde{T}_{i, n}^{*}\right)$ and another pointing from $\left(i-1, \tilde{T}_{i, n}^{*}\right)$ to $\left(i, \tilde{T}_{i, n}^{*}\right)$.

As previously we get a random graph that we denote $\tilde{\mathscr{G}}$. Now we say that a path in $\tilde{\mathscr{G}}$ is a dual-valid path if it satisfies the following constraints:

- it never cross a " $\delta "$ mark,

- when moving upward, we never cross the tip of an arrow.

We write $(i, t) \stackrel{\text { dual }}{\longrightarrow}\left(j, t^{\prime}\right)$ when there is a dual-valid path from $(i, t)$ to $\left(j, t^{\prime}\right)$ in $\mathscr{G}^{\prime}$. Then, for any $A \in \mathscr{P}(\mathbb{Z})$ and for any $t \geq 0$, we can write :

$$
\widetilde{\xi}^{A}(t)=\{j \in \mathbb{Z}:(i, 0) \stackrel{\text { dual }}{\longrightarrow}(j, t) \text { for some } i \in A\}
$$

The same way we defined the finite and semi-infinite processes in the previous sections, we define the finite and semi-infinite dual processes, using the random graph $\tilde{\mathscr{G}}$, and as previously we denote them $\left(\widetilde{\xi}_{[N,+\infty[}(t)\right)_{t \geq 0},\left(\widetilde{\xi}_{-\infty, N]}(t)\right)_{t \geq 0}$ and $\left(\widetilde{\xi}_{N}(t)\right)_{t \geq 0}$. We define their extinction times as well, denoted $\tilde{\tau}_{N}^{A}, \tilde{\tau}_{[N,+\infty[}^{A}$ and $\tilde{\tau}_{-\infty, N]}^{A}$ for any suitable initial configuration $A \in \mathscr{P}(\mathbb{Z})$.

\subsection{Asymptotic behavior}

\subsubsection{Asymptotic behavior of the infinite processes}

The dual process itself presents some kind of phase transition, as stated in the following theorem (which is Theorem 3 in [FGGL18]).

Theorem 3.5.1 There exists $0<\gamma_{c}<+\infty$ such that for all $i \in \mathbb{Z}$ we have:

$$
\mathbb{P}\left(\tilde{\tau}^{i}=+\infty\right)>0, \text { if } \gamma<\gamma_{c}
$$




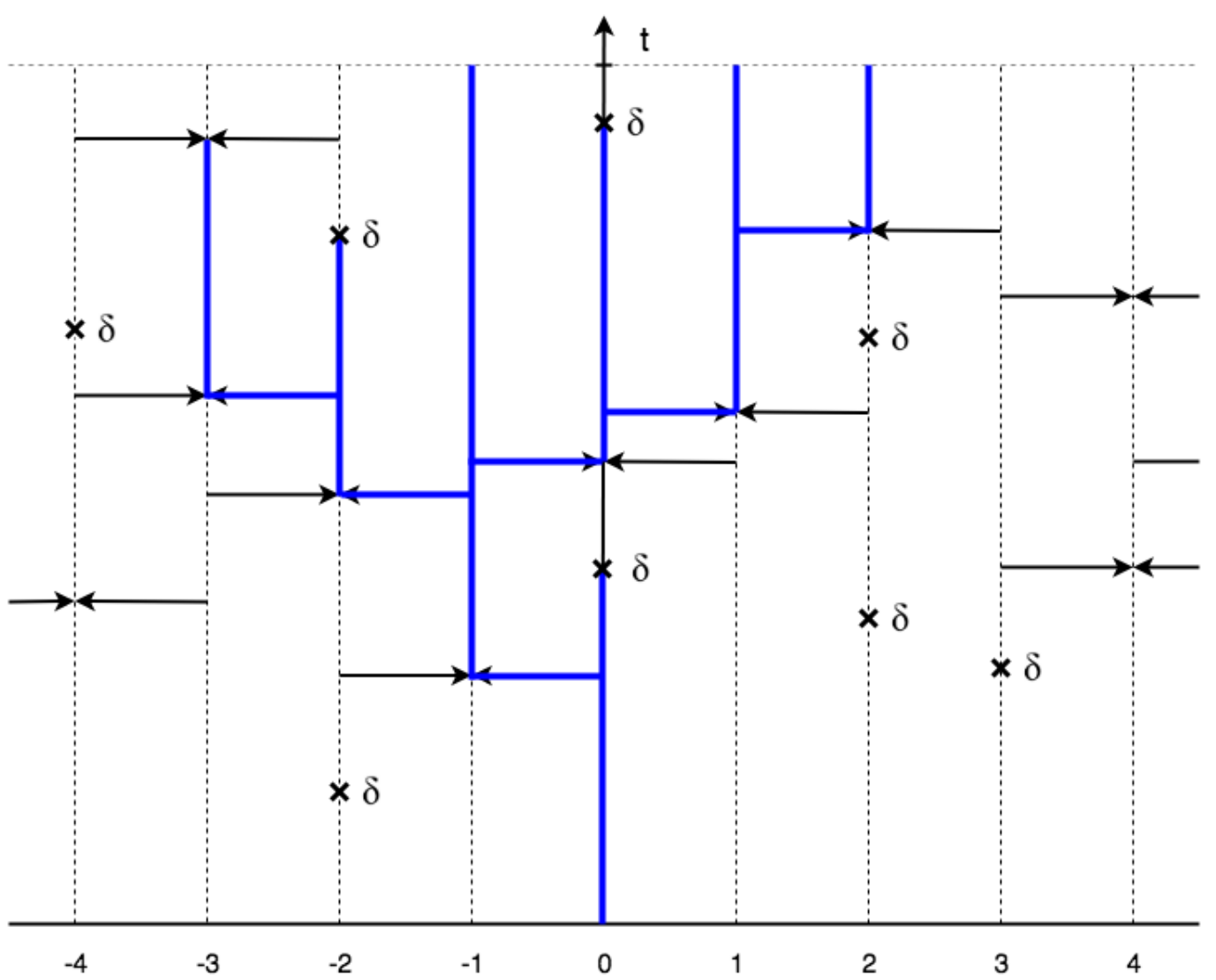

Figure 3.2: A realization of the dual random graph $\mathscr{G}^{\prime}$. In blue all the possible dual-valid paths starting from $(0,0)$. Here the configuration of the dual process at time $t$ when the initial configuration is the singleton $\{0\}$ is the set $\{-1,1,2\}$.

and

$$
\mathbb{P}\left(\tilde{\tau}^{i}=+\infty\right)=0 \text {, if } \gamma>\gamma_{c} .
$$

A central problem we need to address is to determine the invariant measures of each of the different processes we introduced, in the sub-critical regime. Most of this analysis is done by combining Theorem 3.5.1 and the duality property.

Before going any further we begin by giving a topological structure to the state space, which will be needed in what follows. We equip $\{0,1\}$ with the discrete topology so that $\{0,1\}^{\mathbb{Z}}$ can then be equipped with the corresponding product topology. That way $\{0,1\}^{\mathbb{Z}}$ is compact by Tychonoff's theorem and metrizable as any distance of the form $d(x, y)=$ $\sum_{i \in \mathbb{Z}} a_{i} \mathbb{1}_{\{x(i)=y(i)\}}$ generates the product topology (where $\left(a_{i}\right)_{i \in \mathbb{Z}}$ is any sequence satisfying $\left.\sum_{i \in \mathbb{Z}} a_{i}<\infty\right)$. The topological space $\{0,1\}^{\mathbb{Z}}$ is then associated with the corresponding Borel $\sigma$-algebra.

Now let us introduce some order relations on $\{0,1\}^{\mathbb{Z}}$ and on the set of probability measures on $\{0,1\}^{\mathbb{Z}}$ that will be needed in what follows. Given two configurations $\xi^{1}$ and 
$\xi^{2}$, we will say that $\xi^{1} \leq \xi^{2}$ if for any $i \in \mathbb{Z}$ we have $\xi_{i}^{1} \leq \xi_{i}^{2}$. Now, for any continuous function $f$ on $\{0,1\}^{\mathbb{Z}}$, we say that $f$ is increasing if $f\left(\xi^{1}\right) \leq f\left(\xi^{2}\right)$ whenever $\xi^{1} \leq \xi^{2}$. We say that $f$ is decreasing if $-f$ is increasing. Finally, given two probability measures $\nu_{1}$ and $\nu_{2}$ on $\{0,1\}^{\mathbb{Z}}$, we say that $\nu_{1} \leq \nu_{2}$ whenever the following inequality

$$
\int f d \nu_{1} \leq \int f d \nu_{2}
$$

holds for any continuous and increasing function $f$. One of the reasons behind this definition is that it is a well-known fact that the set of continuous and increasing function on $\{0,1\}^{\mathbb{Z}}$ is distribution determining (see Section B.1 in Appendix B), which means that for any probability measures $\nu_{1}$ and $\nu_{2}$ on $\{0,1\}^{\mathbb{Z}}$, if the following equality

$$
\int f d \nu_{1}=\int f d \nu_{2}
$$

holds for any continuous and increasing function $f$ then we have $\nu_{1}=\nu_{2}$. In particular this implies that if $\nu_{1} \leq \nu_{2}$ and $\nu_{1} \geq \nu_{2}$ then $\nu_{1}=\nu_{2}$.

The next result - and therefore most of the results that follow - could be proved using a very general tool called the "basic coupling" (see Theorem 2.4 in Chapter 2 of [LIG85] or section 7 of [DUR81]), nonetheless we give a somewhat more elementary proof based on the graphical construction in order to make this work as self-contained as possible.

Proposition 3.5.2 For any $0 \leq s<t$ we have the following

$$
\mathbb{P}(\xi(s) \in \cdot) \geq \mathbb{P}(\xi(t) \in \cdot) .
$$

Proof: Fix $0 \leq s<t$. Then let $\xi$ be a random variable taking value in $\{0,1\}^{\mathbb{Z}}$ and having the same distribution as $\xi(t-s)$. By the first part of Proposition 3.2.1 we have $\xi(s) \geq \xi^{\xi}(s)$, so that for any continuous and increasing function $f$ we have $f(\xi(s)) \geq f\left(\xi^{\xi}(s)\right)$, and taking the expectation we get $\mathbb{E}(f(\xi(s))) \geq \mathbb{E}\left(f\left(\xi^{\xi}(s)\right)\right)$. But by construction $\xi^{\xi}(s)$ has the same distribution as $\xi(t)$ so that we end up with

$$
\mathbb{E}(f(\xi(s))) \geq \mathbb{E}(f(\xi(t)))
$$

which is the same as

$$
\int f d \mathbb{P}(\xi(s) \in \cdot) \geq \int f d \mathbb{P}(\xi(t) \in \cdot)
$$

Remark 3.5.3 To avoid confusion we call the property of Proposition 3.5.2 stochastic monotonicity, while we will call the first property of Proposition 3.2.1 set monotonicity.

From this last proposition we get the following. 
Corollary 3.5.4 For any $\gamma>0$ there exists a probability measure $\mu_{\gamma}$ that is invariant for $(\xi(t))_{t \geq 0}$ and that is such that

$$
\mathbb{P}(\xi(t) \in \cdot) \underset{t \rightarrow \infty}{\longrightarrow} \mu_{\gamma} .
$$

The Dirac measure on the "all zero" configuration, denoted $\delta_{\emptyset}$, is also invariant and we have

$$
\mathbb{P}\left(\xi^{\emptyset}(t) \in \cdot\right) \underset{t \rightarrow \infty}{\longrightarrow} \delta_{\emptyset} .
$$

Moreover if $\nu$ is any other invariant measure then $\delta_{\emptyset} \leq \nu \leq \mu_{\gamma}$.

Proof: The fact that the process starting from the "all zero" configuration converges weakly to $\delta_{\emptyset}$ is of course entirely trivial as we actually have $\xi^{\emptyset}(t)=\emptyset$ for any $t \geq 0$. For the convergence of the process starting from the "all one" configuration take any continuous and increasing function $f$ and remember that we showed in the proof of the previous proposition that $t \mapsto \mathbb{E}(f(\xi(t)))$ is a decreasing function. It follows that $\mathbb{E}(f(\xi(t)))$ converges to some finite constant when $t$ goes to infinity (remember that $\{0,1\}^{\mathbb{Z}}$ is compact). The set of continuous and increasing functions being distribution determining the result follows.

For the last statement of the proposition suppose we have some invariant measure $\nu$ and write $\left(\xi^{\nu}(t)\right)_{t \geq 0}$ for the process with initial configuration chosen randomly with respect to distribution $\nu$. For any $t \geq 0$ by set monotonicity we have $\xi^{\emptyset}(t) \leq \xi^{\nu}(t) \leq \xi(t)$, so for any continuous and increasing function $f$ we have

$$
\mathbb{E}\left(f\left(\xi^{\emptyset}(t)\right)\right) \leq \mathbb{E}\left(f\left(\xi^{\nu}(t)\right)\right) \leq \mathbb{E}(f(\xi(t)))
$$

or equivalently

$$
\mathbb{P}\left(\xi^{\emptyset}(t) \in \cdot\right) \leq \mathbb{P}\left(\xi^{\nu}(t) \in \cdot\right) \leq \mathbb{P}(\xi(t) \in \cdot) .
$$

The inequality $\delta_{\emptyset} \leq \nu \leq \mu_{\gamma}$ then follows from the convergence results proven above and from the fact that $\mathbb{P}\left(\xi^{\nu}(t) \in \cdot\right)=\nu$ for any $t \geq 0$.

The asymptotic distribution of the process starting from the "all one" configuration will be referred as the upper-invariant measure and the asymptotic distribution of the process starting from the "all zero" configuration will be referred as the lower-invariant measure. The dual process has an upper-invariant measure too, which we denote $\tilde{\mu}_{\gamma}$, and his lowerinvariant measure is also the Dirac $\delta_{\emptyset}$ (to see this it suffices to verify that all the arguments used above work for the dual process as well). Moreover the inequality $\delta_{\emptyset} \leq \nu \leq \tilde{\mu}_{\gamma}$ remains true if $\nu$ is an invariant measure for the dual process.

The fact that the upper-invariant and lower-invariant measures are a lower and upper bound respectively for any invariant measure has the following consequence.

Let define the density of the process $(\xi(t))_{t \geq 0}$ :

$$
\rho_{\gamma}=\mu_{\gamma}\left(\left\{\xi: \xi_{0}=1\right\}\right) .
$$


As a consequence of Theorem 3.5.1 we have the following result for the density.

Proposition 3.5.5 When $\gamma<\gamma_{c}$ we have $\rho_{\gamma}>0$, and therefore $\mu_{\gamma} \neq \delta_{\emptyset}$.

Proof: This follows from duality (Equation 3.6) and can be derived as follows

$$
\begin{aligned}
\rho_{\gamma} & =\lim _{t \rightarrow \infty} \mathbb{P}(\xi(t) \cap\{0\} \neq \emptyset) \\
& =\lim _{t \rightarrow \infty} \mathbb{P}\left(\widetilde{\xi}^{0}(t) \neq \emptyset\right) \\
& =\lim _{t \rightarrow \infty} \mathbb{P}\left(\tilde{\tau}^{0}>t\right) \\
& =\mathbb{P}\left(\tilde{\tau}^{0}=\infty\right),
\end{aligned}
$$

and $\mathbb{P}\left(\tilde{\tau}^{0}=\infty\right)>0$ when $\gamma>\gamma_{c}$.

In order to prove the metastability result we are only interested in the sub-critical regime so that from now on we will just assume that $\gamma<\gamma_{c}$ and omit the dependence in $\gamma$ in the notation, writing simply $\mu$ for $\mu_{\gamma}, \tilde{\mu}$ for $\tilde{\mu}_{\gamma}$, and $\rho$ for $\rho_{\gamma}$. We have the following result, which is the equivalent for the dual process of the second part of Proposition 3.5.5.

Proposition 3.5.6 In the sub-critical regime $\tilde{\mu} \neq \delta_{\emptyset}$.

Proof: We denote by $\xi \equiv 0$ the "all zero" configuration. Then

$$
\begin{aligned}
\tilde{\mu}(\xi \equiv 0) & =\lim _{t \rightarrow \infty} \mathbb{P}(\widetilde{\xi}(t)=\emptyset) \\
& =1-\lim _{t \rightarrow \infty} \mathbb{P}(\widetilde{\xi}(t) \neq \emptyset) \\
& \leq 1-\lim _{t \rightarrow \infty} \mathbb{P}\left(\widetilde{\xi}^{0}(t) \neq \emptyset\right) \\
& =1-\mathbb{P}\left(\tilde{\tau}^{0}=\infty\right),
\end{aligned}
$$

so that $\tilde{\mu}(\xi \equiv 0)<1$.

To state the lemma below let introduce the following notation: $\mathscr{I}$ (resp. $\tilde{\mathscr{I}}$ ) will denote the set of invariant measures of the process $(\xi(t))_{t \geq 0}\left(\right.$ resp. $\left.(\widetilde{\xi}(t))_{t \geq 0}\right)$. We know, by classical theory of Markov processes (see for example proposition 1.8 of chapter 1 in [LIG85]) that $\mathscr{I}$ and $\tilde{\mathscr{I}}$ are convex sets, so that we can define $\mathscr{I}_{e}$ and $\tilde{\mathscr{I}}_{e}$ the set of extreme points of $\mathscr{I}$ and $\tilde{\mathscr{I}}$ respectively, and we know that $\mathscr{I}$ and $\tilde{\mathscr{I}}$ are the convex-hull of $\mathscr{I}_{e}$ and $\tilde{\mathscr{I}}_{e}$ respectively (as a consequence of the Krein-Milman theorem).

Lemma 3.5.7 We have $\left\{\delta_{\emptyset}, \mu\right\} \subset \mathscr{I}_{e}$ and $\left\{\delta_{\emptyset}, \tilde{\mu}\right\} \subset \tilde{\mathscr{I}}_{e}$. In words, the upper-invariant and lower-invariant measures are extremal. 
Proof: We prove the statement for $\mu$. Suppose that there exists $\nu_{1}$ and $\nu_{2}$ in $\mathscr{I}$ such that $\mu=p \nu_{1}+(1-p) \nu_{2}$ for some $0<p<1$. Then Proposition 3.5.4 gives us that $\mu_{1} \leq \mu$ and $\mu_{2} \leq \mu$ so that for any continuous and monotone function $f$ we have

$$
\int f d \nu_{1} \leq \int f d \mu \text { and } \int f d \nu_{2} \leq \int f d \mu
$$

But we also have

$$
\int f d \mu=p \int f d \nu_{1}+(1-p) \int f d \nu_{2}
$$

from what if follows that

$$
\int f d \mu=\int f d \nu_{1}=\int f d \nu_{2}
$$

Using this last lemma it can be shown that not only $\mu$ (as well as $\tilde{\mu}$ ) is different from $\delta_{\emptyset}$ in the sub-critical regime, but it also puts no mass on $\eta \equiv 0$. This is the subject of the following proposition.

Proposition 3.5.8 We have $\mu(\eta \equiv 0)=\tilde{\mu}(\eta \equiv 0)=0$ in the sub-critical regime.

Proof: We prove the statement for $\mu$. Regardless of the value of $\mu(\eta \equiv 0)$ we can always find some $p \in[0,1]$ and some probability measure $\nu$ satisfying $\nu(\eta \equiv 0)=0$ such that

$$
\mu=p \delta_{\emptyset}+(1-p) \nu
$$

We know that $\mu \neq \delta_{\emptyset}$ so that $p$ has to be different from 1 , thus $\nu$ need to be invariant as well. But if $p$ were different from 0 then $\mu$ would be a (non-trivial) convex combination of invariant measures, which would be a contradiction with Lemma 3.5.7. We conclude that $\mu=\nu$.

Finally, one important result we will need in order to prove metastability is the spatial ergodicity of the measure $\mu$. It is stated in the following theorem.

Theorem 3.5.9 The measure $\mu$ is spatially ergodic in the sense that a sequence of random variable $\left(Y_{k}\right)_{k \in \mathbb{Z}}$ taking value in $\{0,1\}$ and such that $Y_{k}$ is distributed like $\mu(\{\xi$ : $\left.\left.\xi_{k}=\cdot\right\}\right)$ would satisfy the following

$$
\frac{1}{n+1} \sum_{k=0}^{n} Y_{k} \underset{n \rightarrow \infty}{\stackrel{a . s}{\rightarrow}} \rho .
$$

Proof: Using a similar coupling as in the proof of Proposition 3.5.2 we can construct an infinite sequence of random variables in $\{0,1\}^{\mathbb{Z}}$, denoted $\left(\xi^{k}\right)_{k \in \mathbb{N} \cup\{\infty\}}$, satisfying $\xi^{0} \geq \xi^{1} \geq$ 
$\xi^{2} \geq \ldots \geq \xi^{\infty}$ and such that $\xi^{0}$ is equal to $\xi \equiv 1$, the "all one" configuration, $\xi^{k}$ has the same distribution as $\xi(k)$ for any $k \geq 0$, and $\xi^{\infty}$ is distributed according to $\mu$. Let $\theta$ be the shift operator on $\{0,1\}^{\mathbb{Z}}$, i.e. the operator defined for any $\xi \in\{0,1\}^{\mathbb{Z}}$ and $i \in \mathbb{Z}$ by $(\theta \xi)_{i}=\xi_{i+1}$. For $k \geq 0$ the composition of order $k$ of $\theta$ with itself will be denoted $\theta^{k}$. Moreover let $\mathbb{1}_{0}$ be the function defined for any $\xi \in\{0,1\}^{\mathbb{Z}}$ by $\mathbb{1}_{0}(\xi)=\mathbb{1}_{\left\{\xi_{0}=1\right\}}$. Then for any $m \geq 0$ and $n \geq 0$ we have

$$
\frac{1}{n+1} \sum_{k=0}^{n} \mathbb{1}_{0}\left(\theta^{k}\left(\xi^{m}\right)\right) \geq \frac{1}{n+1} \sum_{k=0}^{n} \mathbb{1}_{0}\left(\theta^{k}\left(\xi^{\infty}\right)\right) .
$$

For any $t \geq 0$ we know that $\left(\xi_{k}(t)\right)_{k \in \mathbb{Z}}$ is an ergodic stationary sequence (this is true for any system with finite range interaction, see [HOL72] page 1967). Therefore, if we denote by $\mathscr{S}$ the sigma-algebra of shift invariant events with respect to $\mu$ (see Chapter 7 of [DUR10] for precise definitions) then by Birkhoff's ergodic theorem the right-hand side of (3.7) converges to $\mathbb{E}\left(\mathbb{1}_{0}\left(\xi^{\infty}\right) \mid \mathscr{S}\right)$ almost surely when $n$ goes to $\infty$ while the left-hand side converges to $\mathbb{E}\left(\mathbb{1}_{0}\left(\xi^{m}\right)\right)$. It follows that for any $m \geq 0$ we have

$$
\mathbb{E}\left(\mathbb{1}_{0}\left(\xi^{m}\right)\right) \geq \mathbb{E}\left(\mathbb{1}_{0}\left(\xi^{\infty}\right) \mid \mathscr{S}\right) \text { a.s. }
$$

And taking the limit when $m$ goes to $\infty$

$$
\mathbb{E}\left(\mathbb{1}_{0}\left(\xi^{\infty}\right)\right) \geq \mathbb{E}\left(\mathbb{1}_{0}\left(\xi^{\infty}\right) \mid \mathscr{S}\right) \text { a.s. }
$$

But a real-valued random variable which is bounded by its own expectation need to be almost surely equal to it, so that in the end we have

$$
\frac{1}{n+1} \sum_{k=0}^{n} \mathbb{1}_{0}\left(\theta^{k}\left(\xi^{\infty}\right)\right) \underset{n \rightarrow \infty}{\stackrel{a . s .}{\rightarrow}} \mathbb{E}\left(\mathbb{1}_{0}\left(\xi^{\infty}\right)\right),
$$

and it suffices to point out that $\mathbb{E}\left(\mathbb{1}_{0}\left(\xi^{\infty}\right)\right)=\rho$ to end the proof.

\subsubsection{Asymptotic behavior of the semi-infinite processes}

In order to show that the asymptotic behavior of the semi-infinite processes is essentially the same as the asymptotic behavior of the infinite process, we need to make sure that we have an equivalent of Theorem 3.5.1 for the semi-infinite dual process, so that the developments of section 3.5.1 remain valid in the semi-infinite case. This question of whether or not the phase transition remains true, and if it does, with the same critical value, is indeed not trivial. One could indeed imagine that the boundary on the left or on the right somewhat produce a different behavior. Moreover, as it will appear later, it is a crucial point for the proof of Theorem 3.1.1 that the phase transition remains true for the semi-infinite processes. The proof uses a contour argument (see for example [GRI80]) and is somewhat similar to the proof presented in [FGGL18] for the infinite process, nonetheless this former proof is a bit 
elliptical and one of the goals we're pursuing here is to give a clearer argument. We observe that the phase transition for the original semi-infinite process (in the form of Theorem 2.1.2) is a consequence of the Theorem 3.5.11 proven below - which is only concerned with the dual process - using the same standard arguments as in section 4 of [FGGL18]. Nonetheless we don't state this result in this form here as it is unnecessary for our main purpose, which is metastability.

Notice that the following Theorem is stated for the process defined on $\mathscr{P}(\llbracket 0,+\infty \llbracket)$ but that by symmetry it obviously remains true for the process on $\mathscr{P}(\rrbracket-\infty, 0 \rrbracket)$, and more generally for the processes defined on $\mathscr{P}(\llbracket N,+\infty \rrbracket)$ or $\mathscr{P}(\rrbracket-\infty, N \rrbracket)$ for any value of $N \in \mathbb{Z}$.

In order to prove this phase transition, we need a preliminary result of monotonicity in $\gamma$. In the following lemma, we write $\widetilde{\xi}_{[0, \infty[}^{i, \gamma}$ to make explicit the dependence in $\gamma$.

Lemma 3.5.10 For any $i \in \llbracket 0,+\infty \llbracket$ and for any $t \geq 0$ we have the following:

$$
\text { if } \gamma_{1}<\gamma_{2} \text {, then } \mathbb{P}\left(\widetilde{\xi}_{[0, \infty[}^{i, \gamma_{1}}(t)=\emptyset\right) \leq \mathbb{P}\left(\widetilde{\xi}_{[0, \infty[}^{i, \gamma_{2}}(t)=\emptyset\right) \text {. }
$$

Proof: The proof is exactly the same as the proof of Lemma 5 in [FGGL18].

Theorem 3.5.11 There exists $0<\gamma_{c}^{\prime}<+\infty$ such that for all $i \in \mathbb{Z} \cap[0,+\infty]$ we have:

$$
\mathbb{P}\left(\tilde{\tau}_{[0,+\infty[}^{i}=+\infty\right)>0, \text { if } \gamma<\gamma_{c}^{\prime},
$$

and

$$
\mathbb{P}\left(\tilde{\tau}_{[0,+\infty[}^{i}=+\infty\right)=0 \text {, if } \gamma>\gamma_{c}^{\prime} .
$$

Proof: Notice that by Lemma 3.5.10 the function $\gamma \mapsto \mathbb{P}\left(\lim _{t \rightarrow \infty} \widetilde{\xi}_{[0, \infty]}^{0, \gamma}(t)=\emptyset\right)$ is nondecreasing, so that in order to prove the theorem it is sufficient to find two different values of the parameter $\gamma$ such that $\mathbb{P}\left(\tilde{\tau}_{[0,+\infty[}^{i}=+\infty\right)>0$ for any $i \in \mathbb{Z}$ for the first one and $\mathbb{P}\left(\tilde{\tau}_{[0,+\infty[}^{i}=+\infty\right)=0$ for the second one.

The second part of the Theorem is immediate by monotonicity. Indeed, for $\gamma>\gamma_{c}$ (where $\gamma_{c}$ is the critical value for the infinite process) and for any $i \in \mathbb{Z}$

$$
\mathbb{P}\left(\tilde{\tau}_{[0,+\infty[}^{i}=+\infty\right) \leq \mathbb{P}\left(\tilde{\tau}^{i}=+\infty\right)=0
$$

Moreover it has been proven in [FGGL18] that $\gamma_{c}<1$, so that we also have $\gamma_{c}^{\prime}<1$.

To fix ideas, we prove the first part of the Theorem for the process starting at $\{0\}$. The general result will then follow as, for any $i \in \mathbb{Z}^{+}$, we have $\mathbb{P}\left(\tilde{\tau}_{[0,+\infty[}^{i}=+\infty\right) \geq$ $\mathbb{P}\left(\tilde{\tau}_{[0,+\infty[}^{0}=+\infty\right)$ (this can be showed by coupling, using a modification of the graph $\tilde{\mathscr{G}}$ where all events has been shifted spatially to the right $i$ times). 
For the first part of the theorem, we will use the fact that the event $\left\{\tilde{\tau}_{[0,+\infty[}^{0}<\infty\right\}$ is equivalent to the event that $\widetilde{\xi}^{0} \stackrel{\text { def }}{=} \bigcup_{t \geq 0} \widetilde{\xi}_{[0,+\infty}^{0}(t)$ is a finite set. It will therefore be sufficient to prove that $\mathbb{P}\left(\left|\widetilde{\xi}^{0}\right|<\infty\right)<1$. In order to do this we consider a realization of $\left|\widetilde{\xi}^{0}\right|<\infty$ and draw its contour by embedding the time-space diagram $\mathbb{Z} \times \mathbb{R}_{+}$in $\mathbb{R} \times \mathbb{R}_{+}$ and by then defining

$$
E \stackrel{\text { def }}{=}\left\{(y, t):|y-j| \leq \frac{1}{2} \text { for some } j \in \widetilde{\xi}^{i}(t), t \geq 0\right\} .
$$

Now let fill the holes of $E$ to get the set $\tilde{E}$, and let $\Gamma$ be the boundaries of $\tilde{E}$. $\Gamma$ consists of a sequence of alternating horizontal and vertical segments and with a little thought is should be clear that there are exactly $4 n$ of them (for some $n \in \mathbb{Z}$ ). Moreover we encode $\Gamma$ not as a sequence of horizontal and vertical segments but as a sequence of direction triplets $\left(D_{1}, D_{2}, \ldots D_{2 n}\right)$. This is done as follows: start at $\left(\frac{1}{2}, 0\right)$ and follow the boundary of $\Gamma$ in counterclockwise direction, at step $i$ you'll be going trough $D_{i}$, which is one of the seven possible triplets:

$$
\text { uru, ulu, uld, drd, dru, dld, dlu, }
$$

where $u, l, d$, and $r$ means "up", "left", "down", and "right". The last direction of the current triplet is the first direction of the next one.

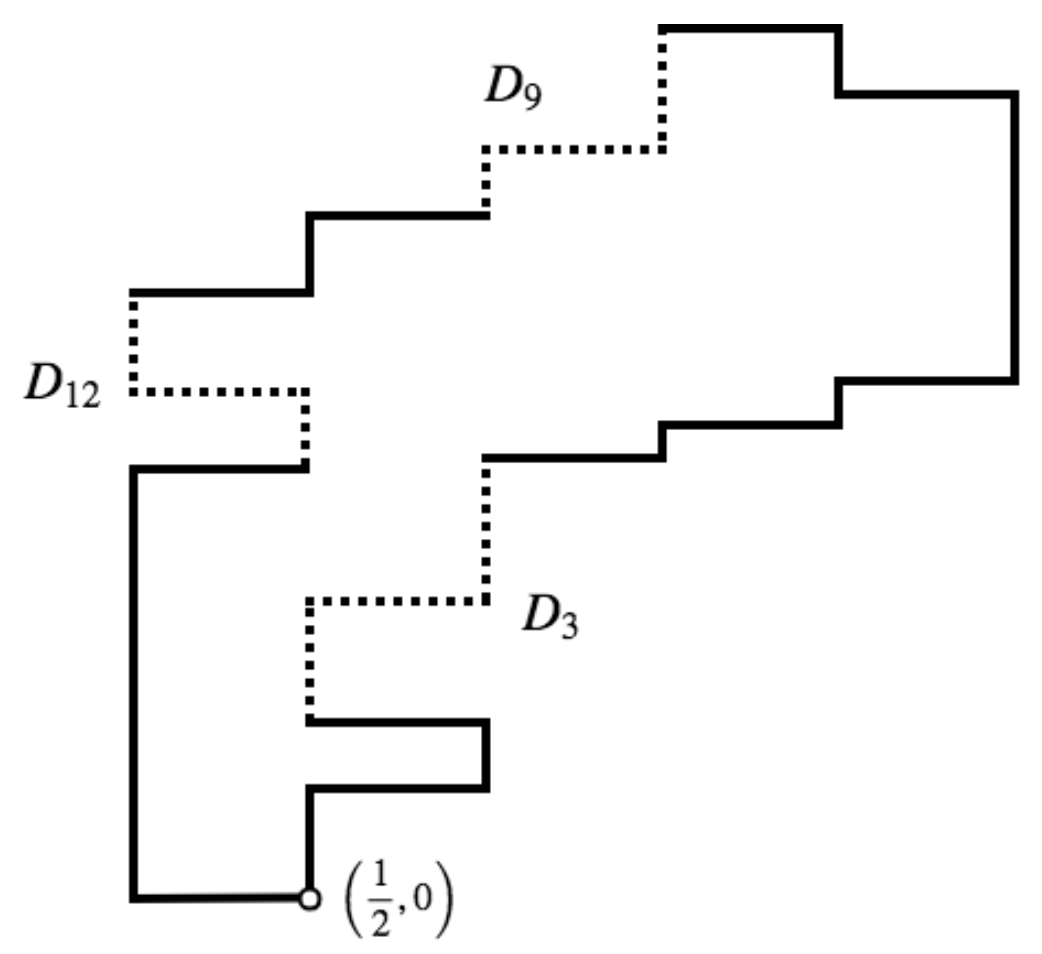

Figure 3.3: Example of a possible contour with $n=7$. Some of the direction vectors are explicitly drawn. 
Now we need to bound the probability of occurrence of some of these events. For reasons that will become clear soon it is sufficient to bound the occurrence of $d l d, d l u$ and $u l u$.

First we find a bound for the probability of occurrence of both $d l d$ and $d l u$. For a given $j \in\{1, \ldots 2 n\}$, let consider what could happen to $D_{j}$. Consider the first horizontal segment which is immediately before the first vertical segment of $D_{j}$ (see figure 3.4 ), there is two possibilities:

- if it is oriented to the left, let $(k, t) \in \mathbb{Z} \times \mathbb{R}_{+}$be the coordinates of the point immediately to the left of its left extremity,

- if it is oriented to the right, let $(k, t) \in \mathbb{Z} \times \mathbb{R}_{+}$be the coordinates of its midpoint.

Notice that there is one possible case in which $k=-1$, when we are hitting the left border of our restricted space-time diagram, but in this particular case the occurrence of a $d l u$ or $d l d$ is not possible, so that we can simply assume that $k \geq 0$. Now let define

$$
F_{j}^{\dagger}=\sup \left\{s \leq t: \tilde{T}_{k, n}^{\dagger}=s, n \geq 0\right\},
$$

and

$$
F_{j}=\sup \left\{s \leq t: \tilde{T}_{k, n}=s, n \geq 0\right\} .
$$

It is not hard to see that (see again figure 3.4)

$$
\left\{D_{j}=d l u\right\} \cup\left\{D_{j}=d l d\right\} \subset\left\{F_{j}^{\dagger} \geq F_{j}\right\} \stackrel{\text { def }}{=} E_{j} .
$$

In words, the occurrence of $d l d$ or $d l u$ implies that when you follow the line $\{k\} \times \mathbb{R}_{+}$ downward starting from $(k, t)$, then the first event you'll encounter is a $\delta$ (see figure 3.4).

Finally, as it doesn't matter for the jump times of a Poisson process whether the time goes upward or downward, we have

$$
\mathbb{P}\left(E_{j}\right)=\frac{\gamma}{1+\gamma} \leq \gamma
$$

It remains to bound the occurrence of ulu. using the same notation as in the precedent cases for the coordinates of the objects considered, let's define

$$
G_{j}^{\dagger}=\sup \left\{s \geq t: \tilde{T}_{k, n}^{\dagger}=s, n \geq 0\right\},
$$

and

$$
G_{j}=\sup \left\{s \geq t: \tilde{T}_{k+1, n}=s, n \geq 0\right\}
$$

Then we have

$$
\left\{D_{j}=u l u\right\} \subset\left\{G_{j}^{\dagger} \leq G_{j}\right\} \stackrel{\text { def }}{=} E_{j}^{\prime} .
$$

Indeed, the occurrence of ulu imply that when you follow simultaneously the two lines $\{k\} \times \mathbb{R}_{+}$and $\{k+1\} \times \mathbb{R}_{+}$upward, starting from $(k, t)$, you'll encounter a $\delta$ on the line 
$\{k\} \times \mathbb{R}_{+}$before you encounter a spike on the line $\{k+1\} \times \mathbb{R}_{+}$(see again figure 3.4 ). Moreover we have

$$
\mathbb{P}\left(E_{j}^{\prime}\right)=\frac{\gamma}{1+\gamma} \leq \gamma
$$
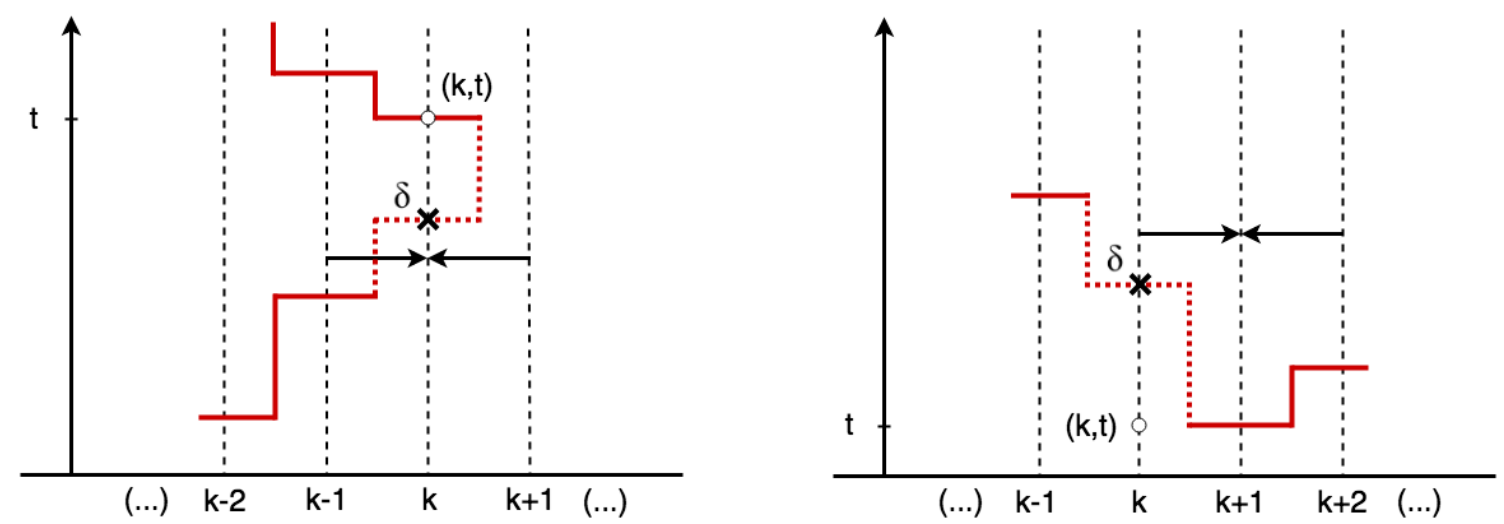

Figure 3.4: On the left side we can see $F_{j}^{\dagger}$ and $F_{j}$ that are represented on the time-space diagram. The $\delta$ corresponds to $F_{j}^{\dagger}$ and the double arrow corresponds to $F_{j} . G_{j}^{\dagger}$ and $G_{j}$ are represented on the right side. The red line represents $\Gamma$ and the dashed part of it represents $D_{j}$, which took value dld on the left, and ulu on the right.

Now we would like to use the mutual independence of all the events $E_{j}$ and $E_{j}^{\prime}$ in order to get a bound for our contour. Unfortunately there is some cases in which these events are actually not independent. Indeed, any event $E_{j}^{\prime}$ (corresponding to an $u l u$ ) makes use of both the deaths occurring on the concerned portion of some line $k \times \mathbb{R}^{+}$and of the double arrows occurring on the concerned portion of the line $(k+1) \times \mathbb{R}^{+}$. However, if the preceding triplet is a $d l u$ (as in the right part of Figure 3.4), then a portion of the line $(k+1) \times \mathbb{R}^{+}$that was used in order to bound the ulu is also used to bound this $d l u$, so that the independence doesn't hold in this specific case. We solve this problem by avoiding contiguity using a partition of the $D_{i}$ 's in two subsets.

A given triplet $D_{j}$ crosses one single line of the form $k \times \mathbb{R}^{+}$. If $k$ is even we say that $D_{j}$ is of type 1 whereas if $k$ is odd we say that $D_{j}$ is of type 2 . That way all $D_{i}$ 's of a given type are non-contiguous (i.e. depend on different Poisson processes or at least on disjoint regions of the same Poisson processes).

Now we write $N(d l d)$ for the number of occurrences of $d l d$ in a given contour, $N(d l u)$ for the number of occurrences of $d l u$ and so on. We also write $N_{1}(d l d)$ and $N_{2}(d l d)$ for the number of $d l d$ of type 1 and type 2 respectively, $N_{1}(d l u)$ and $N_{2}(d l u)$ for $d l u$ and so on.

For a given contour $\Gamma$, from the discussion above and from the fact that we can just discard the occurrences of the other triplets from the intersection of events in which $\Gamma$ consists, it follows that we can bound its probability by

$$
\gamma^{N_{1}(d l d)+N_{1}(d l u)+N_{1}(u l u)},
$$




\section{Chapter 3. Metastability in the sub-critical region for the one-dimensional}

or indifferently by

$$
\gamma^{N_{2}(d l d)+N_{2}(d l u)+N_{2}(u l u)} .
$$

Now notice that $\Gamma$ necessarily contains the same number of left and right oriented segments so that in particular, as $\Gamma$ contains $2 n$ horizontal segments, it shall contain exactly $n$ segments oriented to the left. Thus we have the following equation

$$
N(d l d)+N(d l u)+N(u l d)+N(u l u)=n .
$$

Moreover it is not difficult to see that

$$
N(u l d)=N(d l u)+1
$$

which, together with the previous equation, allows the following bound

$$
N(u l d) \leq \frac{n+1}{2}
$$

It follows that

$$
N(d l d)+N(d l u)+N(u l u) \geq \frac{n-1}{2},
$$

so that we either have

$$
N_{1}(d l d)+N_{1}(d l u)+N_{1}(u l u) \geq \frac{n-1}{4},
$$

or

$$
N_{2}(d l d)+N_{2}(d l u)+N_{2}(u l u) \geq \frac{n-1}{4} .
$$

In both cases we get the following bound

$$
\mathbb{P}(\Gamma) \leq(\sqrt[4]{\gamma})^{n-1}
$$

For $n=1$, the probability of having a contour of length 4 is $\frac{1+\gamma}{2+\gamma}$.

For $n=2$, the 2 possibilities for the shape of $\Gamma$ can be bounded by $\gamma$ as both of them contain at least an $u l u$ or a $d l d$, so that the probability of having a contour of length 8 can be bounded by $2 \gamma$.

Observe that the number of possible triplets for an arbitrary $n$-remembering that a given $\Gamma$ contains $2 n$ triplets - can be bounded by $4^{2 n}=16^{n}$. Indeed the first segment of the first triplet is always oriented upward, and the first segment of any other triplet is determined by the last segment of the previous one, so that the number of possibilities can be roughly bounded by 4 for each of the $D_{j}$ 's. 
Finally, for $\gamma<\frac{1}{16^{4}}$, we get the following bound

$$
\begin{aligned}
\mathbb{P}\left(\tilde{\tau}_{[0,+\infty[}^{0}<\infty\right) & \leq \frac{1+\gamma}{2+\gamma}+2 \gamma+\sum_{n \geq 3} 16^{n}(\sqrt[4]{\gamma})^{n-1} \\
& =\frac{1+\gamma}{2+\gamma}+2 \gamma+16^{3} \sqrt{\gamma} \cdot \frac{16 \sqrt[4]{\gamma}}{1-16 \sqrt[4]{\gamma}}
\end{aligned}
$$

When $\gamma$ goes to 0 the right-hand side of the latter inequality goes to $\frac{1}{2}<1$, from what it follows that $\gamma_{c}^{\prime}>0$.

Having proven the phase transition for the semi-infinite dual process we find ourselves in the same situation as we were for the infinite process at the beginning of section 3.5.1, and it is easy to check that all the arguments given there remain valid in the semi-infinite case. The only results that we really are interested in are the ones concerning the sub-critical regime so let's assume $\gamma<\gamma_{c}^{\prime}$. We have stochastic monotonicity and we can define

$$
\mu_{[0,+\infty[} \stackrel{\text { def }}{=} \lim _{t \rightarrow \infty} \mathbb{P}\left(\xi_{[0,+\infty[}(t) \in \cdot\right)
$$

and

$$
\tilde{\mu}_{[0,+\infty[} \stackrel{\text { def }}{=} \lim _{t \rightarrow \infty} \mathbb{P}\left(\widetilde{\xi}_{[0,+\infty[}(t) \in \cdot\right) .
$$

Moreover $\mu_{[0,+\infty[}$ and $\tilde{\mu}_{[0,+\infty[}$ are extremal invariant, and in the sub-critical regime we have that $\mu_{[0,+\infty[} \neq \delta_{\emptyset}$ and $\tilde{\mu}_{[0,+\infty[} \neq \delta_{\emptyset}$, from what it follows that we actually have

$$
\mu_{[0,+\infty[}(\xi \equiv 0)=0 \quad \text { and } \quad \tilde{\mu}_{[0,+\infty[}(\xi \equiv 0)=0 .
$$

\subsection{Some technical lemmas}

Before entering the discussion about metastability, we establish four lemmas that will be needed in the course of the proof. The first three are only consequences of the nearestneighbors nature of the interaction, even if it might not be immediately clear from the proofs, which entirely rely on the graphical construction. The last one is simply a rigorous statement of an intuitive fact, namely that the more scattered your initial configuration is the higher is the probability to survive. The reader in a hurry might simply skip this part to come back to it later if needed.

Lemma 3.6.1 Define $r_{N}(t)=\max \xi_{N}(t)$ and $l_{N}(t)=\min \xi_{N}(t)$. Then, for any $0 \leq$ $t \leq \tau_{N}$, we have the following

$$
\xi_{N}(t) \cap\left[l_{N}(t), r_{N}(t)\right]=\xi(t) \cap\left[l_{N}(t), r_{N}(t)\right] .
$$


Proof: We only need to show that $\xi(t) \cap\left[l_{N}(t), r_{N}(t)\right] \subset \xi_{N}(t) \cap\left[l_{N}(t), r_{N}(t)\right]$. Let $x \in$ $\xi(t) \cap\left[l_{N}(t), r_{N}(t)\right]$. As $x \in \xi(t)$ there exists $y \in \mathbb{Z}$ such that there is a valid path in the graph $\mathscr{G}$ from $(y, 0)$ to $(x, t)$, and we denote it $P_{y \rightarrow x}$. Now we define the left and right frontiers of the finite process

$$
\partial_{N}^{\text {left }}(t) \stackrel{\text { def }}{=}\left\{\left(l_{N}(s), s\right) \in \mathbb{Z} \times \mathbb{R}_{+}, s \in[0, t]\right\}
$$

and

$$
\partial_{N}^{\text {right }}(t) \stackrel{\text { def }}{=}\left\{\left(r_{N}(s), s\right) \in \mathbb{Z} \times \mathbb{R}_{+}, s \in[0, t]\right\} .
$$

Assume first that $y \notin\{-N, \ldots N\}$. Then the path $P_{y \rightarrow x}$ needs to cross at least one of the frontier. Let $t^{\prime}\left(t^{\prime}<t\right)$ be the last time of crossing and without loss of generality assume that this crossing is a crossing of the left frontier. Then $P_{y \rightarrow x}^{t^{\prime}, t}$ - the restriction of $P_{y \rightarrow x}$ to the time interval $\left[t^{\prime}, t\right]$ - is a valid path from $\left(x^{\prime}, t^{\prime}\right)=\partial_{N}^{\text {left }}\left(t^{\prime}\right)$ to $(x, t)$, but by definition $x^{\prime} \in \xi_{N}\left(t^{\prime}\right)$ so that for some $y^{\prime} \in\{-N, \ldots N\}$ there is a valid path $Q_{y^{\prime} \rightarrow x^{\prime}}$ from $\left(y^{\prime}, 0\right)$ to $\left(x^{\prime}, t^{\prime}\right)$. Finally the concatenation of $Q_{y^{\prime} \rightarrow x^{\prime}}$ and $P_{y \rightarrow x}^{t^{\prime}, t} \rightarrow$ is a valid path from $\left(y^{\prime}, 0\right)$ to $(x, t)$, which prove that $x \in \xi_{N}(t)$. Note that in the two last sentences when we used the expression "valid path" what we really meant is valid path for the finite process.

If $y \in\{-N, \ldots N\}$, then either $P_{y \rightarrow x}$ stays inside the frontiers of the finite process and there is nothing to prove, either it crosses one of the frontiers and the argument is the same as above.

Lemma 3.6.2 Fix some $N \in \mathbb{N}^{*}$ and for some non-empty sets $B \subset \mathbb{Z} \cap[1, N]$ and $C \subset \mathbb{Z} \cap[-N,-1]$ define the stopping times

$$
R_{N}^{B}=\inf \left\{t>0:-N \in \xi_{]-\infty, N]}^{B}(t)\right\}
$$

and

$$
L_{N}^{C}=\inf \left\{t>0: N \in \xi_{[-N, \infty[}^{C}(t)\right\} .
$$

$$
\text { If } \tau_{N}^{B \cup C}>\max \left(R_{N}^{B}, L_{N}^{C}\right) \text {, then for any } t>\max \left(R_{N}^{B}, L_{N}^{C}\right) \text {, }
$$

$$
\xi_{N}(t)=\xi_{N}^{B \cup C}(t)
$$

Proof: The proof is similar to the proof of the previous lemma and relies essentially on the fact that the interaction is between nearest neighbors. We assume $\tau_{N}^{B \cup C}>\max \left(R_{N}^{B}, L_{N}^{C}\right)$ and notice that it implies that both $R_{N}^{B}$ and $L_{N}^{C}$ are finite. What we need to show is that for $t>\max \left(R_{N}^{B}, L_{N}^{C}\right)$ we have $\xi_{N}(t) \subset \xi_{N}^{B \cup C}(t)$.

Let $x \in \xi_{N}(t)$. There exists $y \in \mathbb{Z}$ such that there is a valid path in the graph $\mathscr{G}$ from $(y, 0)$ to $(x, t)$, and we denote it $P_{y \rightarrow x}$. If $y \in B \cup C$ there if of course nothing to prove, so let's assume that $y \notin B \cup C$. Without loss of generality we assume that $y$ belongs to the right part of the graph $\mathbb{Z} \cap[0, N]$. We consider the right frontier of the left part, denoted 
$\partial_{[-N, \infty[}^{C}(t)$, and defined for $t \leq L_{N}^{C}$ as follows

$$
\partial_{[-N, \infty[}^{C}(t) \stackrel{\text { def }}{=}\left\{\left(r_{[-N, \infty[}^{C}(s), s\right) \in \mathbb{Z} \times \mathbb{R}_{+}, s \in[0, t]\right\},
$$

where $r_{[-N, \infty[}^{C}(t) \stackrel{\text { def }}{=} \max \xi_{[-N, \infty[}^{C}(t)$ for any $t \geq 0$.

We write simply $\partial_{[-N, \infty[}^{C}$ for $\partial_{[-N, \infty[}^{C}\left(L_{N}^{C}\right)$. As $L_{N}^{C}$ is finite, $\partial_{[-N, \infty[}^{C}$ goes from $(\max C, 0)$ to $\left(N, L^{C}\right)$. Now, as $y>\max C$ and $x \leq N, P_{y \rightarrow x}$ need to cross the frontier $\partial_{[-N, \infty[}^{C}$ at least once, and as in the previous proof, we let $t^{\prime}$ be the time of the last crossing. Then we can find a valid path $Q_{y^{\prime} \rightarrow x^{\prime}}$ for some $y^{\prime} \in C$, from $\left(y^{\prime}, 0\right)$, to $\left(\partial_{[-N, \infty[}^{C}\left[t^{\prime}\right), t^{\prime}\right)$, and by concatenation with $P_{y}^{t^{\prime}, t} \rightarrow x$ - the restriction of $P_{y \rightarrow x}$ to the time interval $\left[t^{\prime}, t\right]$ - we get a valid path from $\left(y^{\prime}, 0\right)$ to $(x, t)$, which prove that $x \in \xi_{N}^{B \cup C}(t)$.

Lemma 3.6.3 For any $t<\tau_{N}$ we have

$$
\min \xi_{N}(t)=\min \xi_{[-N, \infty[}(t),
$$

and

$$
\max \xi_{N}(t)=\max \xi_{]-\infty, N]}(t)
$$

Proof: Let $t<\tau_{N}$. We prove the lemma only for $\min \xi_{N}(t)$ as the proof for $\max \xi_{N}(t)$ is identical. First notice that by monotonicity we have $\min \xi_{[-N, \infty[}(t) \leq \min \xi_{N}(t)$. Now by definition there exists a path $P_{y \rightarrow x}$ from $(y, 0)$ to $(x, t)=\left(\min \xi_{[-N, \infty[}(t), t\right)$, for some $y \in \mathbb{Z} \cap\left[-N, \infty\left[\right.\right.$. Let $\partial_{N}^{\text {left }}(t)$ be as in the proof of Lemma 3.6.1. If you suppose that $\min \xi_{[-N, \infty[}(t)<\min \xi_{N}(t)$, then $P_{y \rightarrow x}$ needs to cross $\partial_{N}^{\text {left }}(t)$ at least once, so we let $t^{\prime}$ be the last time of crossing. Then as usual there exists a path $Q_{y^{\prime} \rightarrow x^{\prime}}$ for some $y^{\prime} \in\{-N, \ldots, N\}$, from $\left(y^{\prime}, 0\right)$, to $\left(\partial_{N}^{\text {left }}\left(t^{\prime}\right), t^{\prime}\right)$, and by concatenation with $P_{y \rightarrow x}^{t^{\prime}, t}$ - the restriction of $P_{y \rightarrow x}$ to the time interval $\left[t^{\prime}, t\right]$ - we get a valid path from $\left(y^{\prime}, 0\right)$ to $(x, t)$, which is a contradiction.

Lemma 3.6.4 Let $A \subset \mathbb{Z}$, such that $|A|=n$ for some $n \geq 0$. Then

$$
\mathbb{P}\left(\tau^{A}<\infty\right) \leq \mathbb{P}\left(\tau^{\{1, \ldots, n\}}<\infty\right)
$$

Proof: This lemma is the equivalent for our process to part c of Theorem 1.9 in [LIG85] concerning the Contact process, and the proof is quite similar so we will merely sketch it here.

We consider a coupling of $\left(\xi^{\{1, \ldots n\}}(t)\right)_{t \geq 0}$ with two other processes, denoted $\left(\xi^{\prime}(t)\right)_{t \geq 0}$ and $(\xi(t))_{t \geq 0}$. These processes are defined as follows 
- We have $\xi^{\{1, \ldots n\}}(0)=\{1, \ldots n\}$ by definition, and we let $\xi^{\prime}(0)=\xi(0)=A$. Active neurons are paired in increasing order in the three processes.

- Whenever a leakage occurs in $\xi^{\{1, \ldots n\}}(t)$ at some time $t \geq 0$, the corresponding paired neurons are also affected by leakage in $\xi^{\prime}(t)$ and $\xi(t)$.

- When a spike occurs in $\xi^{\{1, \ldots n\}}(t)$ at some site $i \in \mathbb{Z}$ for some $t \geq 0$, the spike is propagated in the paired processes in the following sense: the neuron number $i$ becomes quiescent in all three processes ; if neuron $i-1$ (resp. $i+1$ ) is quiescent in all the processes then it becomes active in all processes, and the newly activated neurons are paired together; if $i-1$ (resp. $i+1$ ) is already active in $\xi^{\{1, \ldots n\}}(t)$ but is quiescent in $\xi(t)$ then a neuron $i-1$ (resp. $i+1$ ) becomes active in $\xi(t)$, but not in $\xi^{\prime}(t)$, and the newly activated neuron then spikes and is affected by leakage effect according to its own independent exponential clocks until further notice; if $i-1$ (resp. $i+1$ ) is not already active in $\xi^{\{1, \ldots n\}}(t)$ but is in $\xi(t)$ then neuron $i-1$ (resp. $i+1$ ) is activated in both $\xi^{\{1, \ldots n\}}(t)$ and $\xi^{\prime}(t)$, and the newly activated neurons are paired together as well as with the neuron that was already active in $\xi(t)$.

With this construction we have that $\left|\xi^{\{1, \ldots n\}}(t)\right|=\left|\xi^{\prime}(t)\right| \leq|\xi(t)|$ for any $t \geq 0$, as whenever a neuron is activated in the two first processes, either it is also activated in $\xi(t)$, either the newly activated neuron is paired with an already supernumerary active neuron in $\xi(t)$. Moreover it is clear that $(\xi(t))_{t \geq 0}$ is distributed like $\left(\xi^{A}(t)\right)_{t \geq 0}$, and the desired result follows.

\subsection{Proof of Theorem 3.1.1}

With this preliminaries completed we can now prove the main result. Note that for reasons related to the way we constructed the proof, even if the theorem is concerned with the time of extinction of the process $\left(\xi_{N}(t)\right)_{t \geq 0}$, the critical value $\gamma_{c}^{\prime}$ for which the theorem is stated and here proved is the critical value for the semi-infinite process (see Theorem 3.5.11).

From the definition of $\left(\xi_{N}(t)\right)_{t \geq 0}$ it is clear that, for any $N \in \mathbb{N}, \mathbb{P}\left(\tau_{N}>t\right)$ is continuous and strictly decreasing in $t$ (putting aside the pathological case $\gamma=0$, in which $\tau_{N}=\infty$ almost surely), so that we can define $\beta_{N}$ the unique value in $\mathbb{R}_{+}$such that

$$
\mathbb{P}\left(\tau_{N}>\beta_{N}\right)=e^{-1} .
$$

The main ingredient of the proof is the Proposition 3.18 below. Theorem 3.1.1 then follows from Corollary 3.7.2 and Proposition 5.2.3, which tell us respectively that $\tau_{N} / \beta_{N}$ converges in distribution to an exponential random variable of mean 1 and that $\mathbb{E}\left(\tau_{N}\right) \underset{N \infty}{\sim}$ $\beta_{N}$. 
Proposition 3.7.1 For any $0<\gamma<\gamma_{c}^{\prime}$ and for any $s, t \geq 0$ the following holds

$$
\lim _{N \rightarrow \infty}\left|\mathbb{P}\left(\frac{\tau_{N}}{\beta_{N}}>s+t\right)-\mathbb{P}\left(\frac{\tau_{N}}{\beta_{N}}>s\right) \mathbb{P}\left(\frac{\tau_{N}}{\beta_{N}}>t\right)\right|=0
$$

Proof: Let $0<\gamma<\gamma_{c}^{\prime}$ and, using the fact that the process is Markovian, observe that

$$
\begin{aligned}
\mathbb{P}\left(\frac{\tau_{N}}{\beta_{N}}>s+t\right)= & \sum_{\substack{A \subseteq\{-N, \ldots N\} \\
A \neq \emptyset}} \mathbb{P}\left(\frac{\tau_{N}}{\beta_{N}}>s+t \mid \xi_{N}\left(\beta_{N} s\right)=A\right) \mathbb{P}\left(\xi_{N}\left(\beta_{N} s\right)=A\right) \\
= & \sum_{\substack{A \subseteq\{-N, \ldots N\} \\
A \neq \emptyset}} \mathbb{P}\left(\frac{\tau_{N}}{\beta_{N}}>s+t \mid \xi_{N}\left(\beta_{N} s\right)=A\right) \mathbb{P}\left(\xi_{N}\left(\beta_{N} s\right)=A, \tau_{N}>\beta_{N} s\right) \\
= & \sum_{\substack{A \subseteq\{-N, \ldots N\} \\
A \neq \emptyset}} \mathbb{P}\left(\frac{\tau_{N}^{A}}{\beta_{N}}>t\right) \mathbb{P}\left(\xi_{N}\left(\beta_{N} s\right)=A, \tau_{N}>\beta_{N} s\right) \\
= & \sum_{\substack{A \subseteq\{-N, \ldots N\} \\
A \neq \emptyset}}\left(\mathbb{P}\left(\frac{\tau_{N}^{A}}{\beta_{N}}>t\right)-\mathbb{P}\left(\frac{\tau_{N}}{\beta_{N}}>t\right)\right) \mathbb{P}\left(\xi_{N}\left(\beta_{N} s\right)=A, \tau_{N}>\beta_{N} s\right) \\
& +\mathbb{P}\left(\frac{\tau_{N}}{\beta_{N}}>s\right) \mathbb{P}\left(\frac{\tau_{N}}{\beta_{N}}>t\right) .
\end{aligned}
$$

Now, for any $b>0$, we define the following subset of $\mathscr{P}(\mathbb{Z})$

$$
F_{b}=\left\{A \in \mathscr{P}(\mathbb{Z}): \frac{|A \cap[-b, 0]|}{b+1}>\frac{\rho}{2}, \frac{|A \cap[0, b]|}{b+1}>\frac{\rho}{2}\right\} .
$$

Here $\rho$ denotes the density of $(\xi(t))_{t \geq 0}$ as defined earlier. This set is the key point of the proof, the idea behind its definition being that, as the process is spatially ergodic, whenever $b$ will be big enough the measure of the set $F_{b}$ will be as close to one as needed.

Now from (3.9), using monotonicity, it follows that 


$$
\begin{aligned}
\left|\mathbb{P}\left(\frac{\tau_{N}}{\beta_{N}}>s+t\right)-\mathbb{P}\left(\frac{\tau_{N}}{\beta_{N}}>s\right) \mathbb{P}\left(\frac{\tau_{N}}{\beta_{N}}>t\right)\right| \\
=\sum_{A \subseteq\{-N, \ldots N\}}\left(\mathbb{P}\left(\frac{\tau_{N}}{\beta_{N}}>t\right)-\mathbb{P}\left(\frac{\tau_{N}^{A}}{\beta_{N}}>t\right)\right) \mathbb{P}\left(\xi_{N}\left(\beta_{N} s\right)=A, \tau_{N}>\beta_{N} s\right) \\
\leq \mathbb{P}\left(\frac{\tau_{N}}{\beta_{N}}>s\right) \mathbb{P}\left(\frac{\tau_{N}}{\beta_{N}}>t\right)-\sum_{A \subseteq\{-N, \ldots N\}} \mathbb{P}\left(\frac{\tau_{N}^{A}}{\beta_{N}}>t\right) \mathbb{P}\left(\xi_{N}\left(\beta_{N} s\right)=A\right) \\
\leq \mathbb{P}\left(\frac{\tau_{N}}{\beta_{N}}>s\right) \mathbb{P}\left(\frac{\tau_{N}}{\beta_{N}}>t\right)-\min _{A \subseteq\{-N, \ldots N\}} \mathbb{P}\left(\frac{\tau_{N}^{A}}{\beta_{N}}>t\right) \mathbb{P}\left(\xi_{N}\left(\beta_{N} s\right) \in F_{b}\right) \\
=\mathbb{P}\left(\frac{\tau_{N}}{\beta_{N}}>s\right)\left[\mathbb{P}\left(\frac{\tau_{N}}{\beta_{N}}>t\right)-\min _{A \subseteq\{-N, \ldots N\}} \mathbb{P}\left(\frac{\tau_{N}^{A}}{\beta_{N}}>t\right)\right] \\
+\min _{A \subseteq\{-N, \ldots N\}} \mathbb{P}\left(\frac{\tau_{N}^{A}}{\beta_{N}}>t\right)\left[P\left(\frac{\tau_{N}}{\beta_{N}}>s\right)-\mathbb{P}\left(\xi_{N}\left(\beta_{N} s\right) \in F_{b}\right)\right] \\
\leq \\
\left.\mathbb{P}\left(\frac{\tau_{N}}{\beta_{N}}>t\right)-\min _{A \subseteq\{-N, \ldots N\}} \mathbb{P}\left(\frac{\tau_{N}^{A}}{\beta_{N}}>t\right)\right]+P\left(\frac{\tau_{N}}{\beta_{N}}>s, \xi_{N}\left(\beta_{N} s\right) \notin F_{b}\right) .
\end{aligned}
$$

From now on let fix $\epsilon>0$. The inequality (3.10) tells us that in order to prove (5.3) it is sufficient to prove that we can find $b=b_{\epsilon}$ and $N_{\epsilon}$ such that, for all $N \geq N_{\epsilon}$,

$$
P\left(\xi_{N}\left(\beta_{N} s\right) \notin F_{b}, \tau_{N}>\beta_{N} s\right)<\epsilon,
$$

and

$$
\mathbb{P}\left(\frac{\tau_{N}}{\beta_{N}}>t\right)-\min _{\substack{A \subseteq\{-N, \ldots N\} \\ A \in F_{b}}} \mathbb{P}\left(\frac{\tau_{N}^{A}}{\beta_{N}}>t\right)<\epsilon
$$

We begin with (3.12). First notice that it is enough to show that there exists $b=b_{\epsilon}$ and $N_{\epsilon}$ such that for all $N \geq N_{\epsilon}$ and all $A \in F_{b}$ we have

$$
\mathbb{P}\left(\frac{\tau_{N}}{\beta_{N}}>t\right)-\mathbb{P}\left(\frac{\tau_{N}^{A}}{\beta_{N}}>t\right)<\epsilon,
$$

and, using monotonicity again, we have 


$$
\mathbb{P}\left(\frac{\tau_{N}}{\beta_{N}}>t\right)-\mathbb{P}\left(\frac{\tau_{N}^{A}}{\beta_{N}}>t\right)=\mathbb{P}\left(\frac{\tau_{N}}{\beta_{N}}>t, \frac{\tau_{N}^{A}}{\beta_{N}} \leq t\right) \leq \mathbb{P}\left(\tau_{N} \neq \tau_{N}^{A}\right)
$$

so that it will be sufficient to bound $\mathbb{P}\left(\tau_{N} \neq \tau_{N}^{A}\right)$.

Now for some big enough $n$, as $\tilde{\mu}$ put no mass on $\xi \equiv 0$, we have that

$$
\tilde{\mu}_{[0,+\infty[}(\{B: B \cap[0, n]=\emptyset\})<\frac{\epsilon}{2} .
$$

We take $b_{1}$ such that $b_{1} \cdot \rho / 2 \geq n$ and choose $N_{1}>b_{1}$. Then for any $A \in F_{b_{1}}$ we have $\left|A \cap\left[0, b_{1}\right]\right| \geq b_{1} \cdot \rho / 2 \geq n$, so using Lemma 3.6.4 we get that for any $A \in F_{b_{1}}$ and for any $N \geq N_{1}$

$$
\mathbb{P}\left(\tau_{[-N, \infty[}^{A \cap\left[0, b_{1}\right]}<\infty\right) \leq \mathbb{P}\left(\tau_{[-N, \infty[}^{\{-N, \ldots,-N+n\}}<\infty\right)
$$

and by duality we have

$$
\begin{aligned}
\mathbb{P}\left(\tau_{[-N, \infty[}^{\{-N, \ldots,-N+n\}}=\infty\right) & =\lim _{t \rightarrow \infty} \mathbb{P}\left(\xi_{[-N, \infty[}^{\{-N, \ldots,-N+n\}}(t) \neq \emptyset\right) \\
& =\lim _{t \rightarrow \infty} \mathbb{P}\left(\widetilde{\xi}_{[-N, \infty[}(t) \cap\{-N, \ldots,-N+n\} \neq \emptyset\right) \\
& =\tilde{\mu}_{[0,+\infty[}(B: B \cap[0, n] \neq \emptyset),
\end{aligned}
$$

which proves that, for the $b_{1}$ and $N$ we chose,

$$
\mathbb{P}\left(\tau_{[-N, \infty[}^{A \cap\left[0, b_{1}\right]}<\infty\right)<\frac{\epsilon}{2} .
$$

With the same arguments we also get that

$$
\mathbb{P}\left(\tau_{]-\infty, N]}^{A \cap\left[-b_{1}, 0\right]}<\infty\right)<\frac{\epsilon}{2} .
$$

This leads us to define the following event

$$
E \stackrel{\text { def }}{=}\left\{\tau_{[-N, \infty[}^{A \cap\left[0, b_{1}\right]}=\infty, \tau_{]-\infty, N]}^{A \cap\left[-b_{1}, 0\right]}=\infty\right\} .
$$

We also define the stopping time

$$
U=\max \left(L_{N}^{A \cap\left[-b_{1}, 0\right]}, R_{N}^{A \cap\left[0, b_{1}\right]}\right),
$$

where $L_{N}^{A \cap\left[-b_{1}, 0\right]}$ and $R_{N}^{A \cap\left[0, b_{1}\right]}$ are as in Lemma 3.6.2.

Then, on $E$ we have $\tau_{N}^{A} \geq \tau_{N}^{A \cap\left[-b_{1}, b_{1}\right]} \geq U$, and from Lemma 3.6.2 it follows that, for $t>U$, we have $\xi_{N}(t)=\xi_{N}^{A \cap\left[-b_{1}, b_{1}\right]}(t)$. By monotonicity we have as well for any $t>U$ 


$$
\xi_{N}(t)=\xi_{N}^{A}(t)
$$

Therefore, on $E$, we have $\tau_{N}=\tau_{N}^{A}$.

Now, using this last remark as well as (3.14) and (3.15), we get

$$
\mathbb{P}\left(\tau_{N} \neq \tau_{N}^{A}\right) \leq \mathbb{P}\left(\left\{\tau_{N} \neq \tau_{N}^{A}\right\} \cap E\right)+\mathbb{P}\left(E^{c}\right)<\epsilon,
$$

which gives a final point to the proof of (3.12).

It remains to prove (3.11). For any choice of $b, N$ and $L$ that satisfies the following condition:

$$
b<N-L<N
$$

we have

$$
\begin{aligned}
& P\left(\xi_{N}\left(\beta_{N} s\right) \notin F_{b}, \tau_{N}>\beta_{N} s\right) \\
& \leq \mathbb{P}\left(\xi_{N}\left(\beta_{N} s\right) \notin F_{b}, \tau_{N}>\beta_{N} s, \min \xi_{N}\left(\beta_{N} s\right)<-N+L, \max \xi_{N}\left(\beta_{N} s\right)>N-L\right) \\
& \quad+\mathbb{P}\left(\min \xi_{N}\left(\beta_{N} s\right) \geq-N+L, \tau_{N}>\beta_{N} s\right) \\
& \quad+\mathbb{P}\left(\max \xi_{N}\left(\beta_{N} s\right) \leq N-L, \tau_{N}>\beta_{N} s\right) .
\end{aligned}
$$

For the first term in the summation in (3.17) we have

$$
\begin{aligned}
& \mathbb{P}\left(\xi_{N}\left(\beta_{N} s\right) \notin F_{b}, \tau_{N}>\beta_{N} s, \min \xi_{N}\left(\beta_{N} s\right)<-N+L, \max \xi_{N}\left(\beta_{N} s\right)>N-L\right) \\
& \leq \mathbb{P}\left(\xi_{N}\left(\beta_{N} s\right) \notin F_{b}, \tau_{N}>\beta_{N} s, \min \xi_{N}\left(\beta_{N} s\right)<-b, \max \xi_{N}\left(\beta_{N} s\right)>b\right)
\end{aligned}
$$

so that using Lemma 3.6.1 this term can be bounded by $\mathbb{P}\left(\xi\left(\beta_{N} s\right) \notin F_{b}\right)$.

By the spatial ergodicity of $\mu$ (Theorem 3.5.9) we have $\mu\left(F_{b}^{c}\right) \underset{b \rightarrow \infty}{\longrightarrow} 0$. From this, and using the stochastic monotonicity (Proposition 3.5.2), it follows that we can find $b_{2}$ such that for any $b>b_{2}$

$$
\mathbb{P}\left(\xi\left(\beta_{N} s\right) \notin F_{b}\right)<\frac{\epsilon}{3} .
$$

From Lemma 3.6 .3 we have for any $N$ and $L$

$$
\mathbb{P}\left(\min \xi_{N}\left(\beta_{N} s\right) \geq-N+L, \tau_{N}>\beta_{N} s\right) \leq \mathbb{P}\left(\min \xi_{[-N, \infty[}\left(\beta_{N} s\right) \geq-N+L\right)
$$


But by monotone convergence once again we have

$$
\begin{aligned}
& \mathbb{P}\left(\min \xi_{[-N, \infty[}\left(\beta_{N} s\right) \geq-N+L\right) \\
& \leq \mu_{[-N,+\infty}(\{A \subset[-N, \infty[\cap \mathbb{Z}: A \cap[-N,-N+L-1]=\emptyset\}) \\
& =\mu_{[0,+\infty[}(\{A \subset[0, \infty[\cap \mathbb{Z}: A \cap[0, L-1]=\emptyset\}) .
\end{aligned}
$$

And the later will be arbitrarily close to 0 for arbitrarily big $L$. We therefore fix some big enough $L$ and then fix some $N_{2}$ such that condition (3.16) is satisfied (which in our case means $\left.\max \left(b_{1}, b_{2}\right)<N_{2}-L<L\right)$ and for any $N \geq N_{2}$ we get that

$$
\mathbb{P}\left(\min \xi_{N}\left(\beta_{N} s\right) \geq-N+L, \tau_{N}>\beta_{N} s\right)<\frac{\epsilon}{3} .
$$

With the same arguments and by symmetry the last term in (3.17) is also bounded by $\epsilon / 3$ for $N \geq N_{2}$ and for the same choice of $L$. To finish of course we take $b_{\epsilon}=\max \left(b_{1}, b_{2}\right)$ and $N_{\epsilon}=\max \left(N_{1}, N_{2}\right)$ and both (3.12) and (3.11) are satisfied.

From Proposition 3.18 we obtain the following corollary.

Corollary 3.7.2 For any $0<\gamma<\gamma_{c}^{\prime}$ the following convergence holds

$$
\frac{\tau_{N}}{\beta_{N}} \underset{N \rightarrow \infty}{\stackrel{\mathscr{D}}{\longrightarrow}} \mathscr{E}(1)
$$

Proof: This result follows from the definition of $\beta_{N}$ and a simple density argument. Taking $s=t=1$ in Proposition 3.18 we have

$$
\lim _{N \rightarrow \infty} \mathbb{P}\left(\frac{\tau_{N}}{\beta_{N}}>2\right)=e^{-2}
$$

Iterating this argument we easily obtain that for any $n \in \mathbb{N}$

$$
\lim _{N \rightarrow \infty} \mathbb{P}\left(\frac{\tau_{N}}{\beta_{N}}>2^{n}\right)=e^{-2^{n}}
$$

Moreover, taking $s=t=2^{-1}$ in Proposition 3.18 we have

$$
\lim _{N \rightarrow \infty} \mathbb{P}\left(\frac{\tau_{N}}{\beta_{N}}>2^{-1}\right)=e^{-2^{-1}}
$$




\section{Chapter 3. Metastability in the sub-critical region for the one-dimensional}

And iterating this argument once again we have for any $n \in \mathbb{N}$

$$
\lim _{N \rightarrow \infty} \mathbb{P}\left(\frac{\tau_{N}}{\beta_{N}}>2^{-n}\right)=e^{-2^{-n}}
$$

Now for any $n \in \mathbb{N}$ consider the set $B_{n}$ of the real numbers which binary expansion contains exactly $n$ times the digit 1 , that is:

$$
B_{n} \stackrel{\text { def }}{=}\left\{x \in \mathbb{R}: x=\sum_{k \in \mathbb{Z}} a_{k} 2^{k}, \text { where }\left(a_{k}\right)_{k \in \mathbb{Z}} \in\{0,1\}^{\mathbb{Z}} \text { is such that } \sum_{k \in \mathbb{Z}} a_{k}=n\right\} .
$$

We can then reformulate equations (3.19) and (3.20) as:

$$
\lim _{N \rightarrow \infty} \mathbb{P}\left(\frac{\tau_{N}}{\beta_{N}}>t\right)=e^{-t} \quad \text { for any } t \in B_{1} .
$$

By induction we then easily get (using Proposition 3.18) that for any $n \in \mathbb{N}$

$$
\lim _{N \rightarrow \infty} \mathbb{P}\left(\frac{\tau_{N}}{\beta_{N}}>t\right)=e^{-t} \quad \text { for any } t \in B_{n} .
$$

Now notice that any real number has a binary expansion, so that it can be easily approximated-by below or by above indifferently — by a sequence in $\bigcup_{n \in \mathbb{N}} B_{n}$. Then the result follows by monotonicity of the function $t \mapsto \mathbb{P}\left(\frac{\tau_{N}}{\beta_{N}}>t\right)$.

It only remains to prove that we can replace $\beta_{N}$ by $\mathbb{E}\left(\tau_{N}\right)$ in Corollary 3.7.2. It follows from the proposition below.

Proposition 3.7.3 The following convergence holds

$$
\lim _{N \rightarrow \infty} \frac{\mathbb{E}\left(\tau_{N}\right)}{\beta_{N}}=1
$$

Proof: We know from (3.9) and by monotonicity that for any $s, t \geq 0$ we have

$$
\mathbb{P}\left(\frac{\tau_{N}}{\beta_{N}}>s+t\right) \leq \mathbb{P}\left(\frac{\tau_{N}}{\beta_{N}}>s\right) \mathbb{P}\left(\frac{\tau_{N}}{\beta_{N}}>t\right),
$$

so that it follows from the definition of $\beta_{N}$ that for any integer $n$ we have

$$
\mathbb{P}\left(\frac{\tau_{N}}{\beta_{N}}>n\right) \leq e^{-n}
$$


In general for any $t \geq 0$ we therefore have

$$
\mathbb{P}\left(\frac{\tau_{N}}{\beta_{N}}>t\right) \leq e^{-\lfloor t\rfloor}
$$

Moreover we have

$$
\frac{\mathbb{E}\left(\tau_{N}\right)}{\beta_{N}}=\int_{0}^{\infty} \mathbb{P}\left(\frac{\tau_{N}}{\beta_{N}}>t\right) d t,
$$

so that finally — by Dominated Convergence Theorem and Proposition 3.7.2 — we get

$$
\lim _{N \rightarrow \infty} \frac{\mathbb{E}\left(\tau_{N}\right)}{\beta_{N}}=\int_{0}^{\infty} \lim _{N \rightarrow \infty} \mathbb{P}\left(\frac{\tau_{N}}{\beta_{N}}>t\right) d t=\int_{0}^{\infty} e^{-t} d t=1
$$


Chapter 3. Metastability in the sub-critical region for the one-dimensional 46

lattice process 


\section{Chapter 4}

\section{Asymptotically deterministic time of extinction in the super-critical region for the one-dimensional lattice process}

\subsection{Asymptotically deterministic time of extinction}

In this chapter we continue to consider the process defined on the one-dimensional lattice and our aim is to prove that the main result of the previous chapter, that is to say the convergence of the renormalized time of extinction to an exponential random variable (Theorem 3.1.1) doesn't hold anymore if $\gamma$ is taken big enough. We actually prove the following result.

Theorem 4.1.1 Suppose that $\gamma>1$. Then the following convergence holds

$$
\frac{\tau_{N}}{\mathbb{E}\left(\tau_{N}\right)} \underset{N \rightarrow \infty}{\stackrel{\mathbb{P}}{\longrightarrow}} 1
$$

where $\mathbb{P}$ denotes a convergence in probability.

This result is in some sense symmetrical to Theorem 3.1.1. Indeed the later tells us that in a portion of the sub-critical region the time of extinction is asymptotically memoryless, which means that it is highly unpredictable: knowing that the process survived up to time $t$ doesn't give you any information about what should happen after time $t$. What we prove here is that in a portion of the super-critical regime (indeed $\gamma_{c}<1$ as proven in in [FGGL18]) the time of extinction is asymptotically constant, so that it is highly predictable.

In order to establish this result, we will prove two propositions, from which Theorem 4.1.1 will follow immediately. We first prove that the renormalized time of extinction evolves asymptotically like a logarithm, which is the object of the following proposition. 
Proposition 4.1.2 Suppose that $\gamma>1$. There exists a constant $0<C<\infty$ depending on $\gamma$ such that the following convergence holds

$$
\frac{\tau_{N}}{\log (2 N+1)} \underset{N \rightarrow \infty}{\stackrel{\mathbb{P}}{\longrightarrow}} C .
$$

Then, we prove that the same is true for the expectation.

Proposition 4.1.3 Suppose that $\gamma>1$. Then the following convergence holds

$$
\frac{\mathbb{E}\left(\tau_{N}\right)}{\log (2 N+1)} \underset{N \rightarrow \infty}{\longrightarrow} C,
$$

where $C$ is the same constant as in Proposition 4.1.2.

In the following we adopt the same notation and conventions as in the previous chapter.

\subsection{Proof of Theorem 4.1.1}

\subsubsection{Proof of Proposition 4.1.2}

Our proof uses some of the ideas developed in [DL88] concerning the Harris contact process. We define the following function

$$
t \mapsto f(t)=\log \left(\mathbb{P}\left(\xi^{0}(t) \neq \emptyset\right)\right) .
$$

We also define the following constant

$$
C^{\prime}=-\sup _{s>0} \frac{f(s)}{s} .
$$

The first step is to show that the function $f$ is superadditive. For any $s, t \geq 0$ we have:

$$
\mathbb{P}\left(\xi^{0}(t+s) \neq \emptyset \mid \xi^{0}(t) \neq \emptyset\right) \geq \mathbb{P}\left(\xi^{0}(s) \neq \emptyset\right) .
$$

This last inequality follows from Markov property and the fact that having a higher number of active neurons in the initial configuration implies having a higher probability of being alive for the process at any given time $s$ (see Proposition 4.2 in [? ]). Moreover if $\xi^{0}(t) \neq \emptyset$ then $\left|\xi^{0}(t)\right| \geq 1=\left|\xi^{0}(0)\right|$. Furthermore it can be rewritten as follows

$$
\mathbb{P}\left(\xi^{0}(t+s) \neq \emptyset\right) \geq \mathbb{P}\left(\xi^{0}(t) \neq \emptyset\right) \mathbb{P}\left(\xi^{0}(s) \neq \emptyset\right),
$$

and taking the $\log$ gives the superaddtivity we are looking for. Now from a well-known result about superadditive functions, sometimes called the Fekete lemma (see Section B.2 
in Appendix B), we get the following convergence

$$
\frac{f(t)}{t} \underset{t \rightarrow \infty}{\longrightarrow}-C^{\prime}
$$

For any $t>0$ we also have

$$
\mathbb{P}\left(\xi^{0}(t) \neq \emptyset\right) \leq e^{-C^{\prime} t} .
$$

Notice that while it is clear that $0 \leq C^{\prime}<\infty$, it is not obvious that $C^{\prime}>0$. We show that it is the case using a coupling with a continuous time branching process having birth rate 1 and death rate $\gamma$ (see Section B.3 in B). Let $\left(Z_{t}\right)_{t \geq 0}$ represent the number of individual in the population of this branching process, with $Z_{0}=1$. The expectation at time $t$ can be explicitly computed. For any $\gamma \geq 0$ and $t \geq 0$ we have

$$
\mathbb{E}\left(Z_{t}\right)=e^{-(\gamma-1) t}
$$

The coupling is done as follows, at time 0 the only active neuron in $\xi^{0}(0)$ is coupled with the only individual in $Z(0)$. By this we mean that if this neuron becomes quiescent then the individual dies, and if the neuron spikes, then the individual is replaced by two new individuals. When a spike occurs, there is three possibilities: two neurons are activated, one neuron is activated, or no neuron is activated, depending on how much neighbors are quiescent. In any of these cases each activated neuron is coupled with a newborn individuals, and any supernumerary newborn individual is given his own independent exponential clocks. At any time $t \geq 0$ we obviously have $\left|\xi_{t}^{0}\right| \leq Z_{t}$. Using (4.3) and Markov inequality it follows that

$$
\mathbb{P}\left(\xi^{0}(t) \neq \emptyset\right) \leq \mathbb{P}\left(Z_{t} \geq 1\right) \leq e^{-(\gamma-1) t} .
$$

Then we take the $\log$ and divide by $t$ in the previous inequality and we obtain at the limit that $C^{\prime} \geq \gamma-1$, and from the assumption that $\gamma>1$ we get $C^{\prime}>0$.

Let us break the suspense and already reveal that the constant $C$ we are looking for is actually simply the inverse of $C^{\prime}$. Therefore in order to prove our result we are going to prove that for any $\epsilon>0$ we have the two following convergences

$$
\mathbb{P}\left(\frac{\tau_{N}}{\log (2 N+1)}-\frac{1}{C^{\prime}}>\epsilon\right) \underset{N \rightarrow \infty}{\longrightarrow} 0
$$

and

$$
\mathbb{P}\left(\frac{\tau_{N}}{\log (2 N+1)}-\frac{1}{C^{\prime}}<-\epsilon\right) \underset{N \rightarrow \infty}{\longrightarrow} 0 .
$$

Let us start with (4.4), which is the easiest part. Using additivity (Proposition 3.2.1) 
and inequality (4.2) we get

$$
\mathbb{P}\left(\xi_{N}(t) \neq \emptyset\right) \leq(2 N+1) \mathbb{P}\left(\xi^{0}(t) \neq \emptyset\right) \leq(2 N+1) e^{-C^{\prime} t} .
$$

Now, for any $\epsilon>0$, if you let $t=\left(\frac{1}{C^{\prime}}+\epsilon\right) \log (2 N+1)$ then the following holds

$$
\mathbb{P}\left(\frac{\tau_{N}}{\log (2 N+1)}-\frac{1}{C^{\prime}}>\epsilon\right)=P\left(\xi_{N}(t) \neq \emptyset\right) \leq e^{-C^{\prime} \epsilon \log (2 N+1)} .
$$

Then the fact that $C^{\prime}>0$ ensures us that the term on the right-hand side of the inequality goes to 0 as $N$ diverges, which proves (4.4).

It remains to prove (4.5). If for some $N \in \mathbb{N}^{*}$ we take $t=\left(\frac{1}{C^{\prime}}-\epsilon\right) \log (2 N+1)$, then we can write

$$
\mathbb{P}\left(\frac{\tau_{N}}{\log (2 N+1)}-\frac{1}{C^{\prime}}<-\epsilon\right)=\mathbb{P}\left(\xi_{N}(t)=\emptyset\right),
$$

so that it suffices to show that the right-hand side converges to 0 for this choice of $t$ as $N$ goes to $\infty$. For reasons that will become clear in a moment we will actually write $t=\frac{1}{C^{\prime}}\left(1-\epsilon^{\prime}\right) \log (2 N+1)$, with $\epsilon^{\prime}=C^{\prime} \epsilon$.

From (4.1) (and from the fact that $C^{\prime}>0$ ) we get that for any $\epsilon>0$ and for big enough

$$
\frac{f(t)}{t} \geq-\left(1+\epsilon^{\prime}\right) C^{\prime}
$$

which can be written

$$
\mathbb{P}\left(\xi_{t}^{0}=\emptyset\right) \leq 1-e^{-\left(1+\epsilon^{\prime}\right) C^{\prime} t} .
$$

Therefore, with $t=\frac{1}{C^{\prime}}\left(1-\epsilon^{\prime}\right) \log (2 N+1)$ and $N$ big enough we have

$$
\mathbb{P}\left(\xi_{t}^{0}=\emptyset\right) \leq 1-\frac{1}{(2 N+1)^{1-\epsilon^{\prime 2}}} .
$$

Now for any $k \in \mathbb{Z}$ we define

$$
F_{k} \stackrel{\text { def }}{=} \llbracket(2 k-1) K \log (2 N+1),(2 k+1) K \log (2 N+1) \rrbracket,
$$

where $K$ is some constant depending on $N$ which value will be chosen later in order for $K \log (2 N+1)$ to be an integer. We then consider a modification of the process $\left(\xi_{N}(t)\right)_{t>0}$ where all neurons at the border of one of the sub-windows $F_{k}$ defined above (i.e. all neurons indexed by $(2 k+1) K \log (2 N+1)$ for some $k \in \mathbb{Z})$ are fixed in quiescent state and therefore are never allowed to spike. This modified process is denoted $\left(\bar{\xi}_{N}(t)\right)_{t \geq 0}$. Notice that the fact that the neurons at the borders of the windows $F_{k}$ are never allowed to spike makes 
the evolution of $\left(\bar{\xi}_{N}(t)\right)_{t \geq 0}$ independent from one window to another. We also define the following configuration

$$
A_{N} \stackrel{\text { def }}{=}\left\{2 k K \log (2 N+1) \text { for } k \in \mathbb{Z} \cap\left[-\frac{N}{2 K \log (2 N+1)}, \frac{N}{2 K \log (2 N+1)}\right]\right\} .
$$

In words $A_{N}$ is a finite configuration in which the only active sites are the ones at the center of the $F_{k}$ windows. We refer to Figure 4.1 for a graphical representation of our construction.

Now for any $t \geq 0$ we define $r_{t} \stackrel{\text { def }}{=} \max \xi_{t}^{0}$. Considering the spiking process $\left(\xi_{t}\right)_{t \geq 0}$ with no leaking it is easy to see that the right edge $r_{t}$ can be coupled with an homogeneous Poisson process of parameter 1 , that we denote $(M(t))_{t \geq 0}$, in such a way that for any $m \geq 0$

$$
\mathbb{P}\left(\sup _{s \leq t} r_{s} \geq m\right) \leq \mathbb{P}(M(t) \geq m)
$$

We have

$$
\mathbb{E}\left(e^{M(t)}\right)=e^{t(e-1)},
$$

so taking the exponential, using Markov inequality and taking $m=K^{\prime} t$ (where $K^{\prime}$ is some constant that we are going to fix in a moment) we get

$$
\begin{aligned}
\mathbb{P}\left(\sup _{s \leq t} r_{s} \geq K^{\prime} t\right) & \leq e^{t\left(e-1-K^{\prime}\right)} \\
& \leq e^{t\left(2-K^{\prime}\right)}
\end{aligned}
$$

where in the last inequality we simply used the fact that $e-1<2$.

Now taking again $t=\frac{1}{C^{\prime}}\left(1-\epsilon^{\prime}\right) \log (2 N+1)$ and $K^{\prime}=2\left(1+C^{\prime}\right)$ we get

$$
\mathbb{P}\left(\sup _{s \leq t} r_{s} \geq m\right) \leq e^{-2\left(1-\epsilon^{\prime}\right) \log (2 N+1)},
$$

and assuming without loss of generality that $\epsilon^{\prime}<\frac{1}{2}$ we get

$$
\mathbb{P}\left(\sup _{s \leq t} r_{s} \geq m\right) \leq \frac{1}{2 N+1} .
$$

It is now possible to fix the value of the constant $K$ we introduced earlier. We take

$$
K=\inf \left\{x \in \mathbb{R} \text { such that } x \geq \frac{K^{\prime}}{C^{\prime}} \text { and } x \log (2 N+1) \in \mathbb{N}\right\}
$$


In words we take $K$ equal to $\frac{K^{\prime}}{C^{\prime}}$ and then enlarge it slightly in order for $K \log (2 N+1)$ to be an integer. We also define the following event

$$
E_{t} \stackrel{\text { def }}{=}\left\{\xi_{s}^{0} \text { doesn't escape from } F_{0} \text { for any } s \leq t\right\}
$$

Now taking $N$ large enough and $t=\frac{1}{C^{\prime}}\left(1-\epsilon^{\prime}\right) \log (2 N+1)$ we have

$$
\begin{aligned}
\mathbb{P}\left(\xi_{N}(t)=\emptyset\right) & \leq \mathbb{P}\left(\bar{\xi}_{N}^{A_{N}}(t)=\emptyset\right) \\
& =\mathbb{P}\left(\bar{\xi}_{N}^{0}(t)=\emptyset\right)^{(2 N+1) /(2 K \log (2 N+1))} \\
& \leq\left(\mathbb{P}\left(\bar{\xi}_{N}^{0}(t)=\emptyset \cap E_{t}\right)+\mathbb{P}\left(E_{t}^{c}\right)\right)^{(2 N+1) /(2 K \log (2 N+1))} \\
& \leq\left(\mathbb{P}\left(\xi^{0}(t)=\emptyset\right)+\mathbb{P}\left(E_{t}^{c}\right)\right)^{(2 N+1) /(2 K \log (2 N+1))} \\
& \leq\left(1-\left(\frac{1}{(2 N+1)^{1-\epsilon^{\prime 2}}}-\frac{2}{2 N+1}\right)\right)^{(2 N+1) /(2 K \log (2 N+1))}
\end{aligned}
$$

To obtain the inequality above we used (4.7) and the fact that inside $E_{t}$ the process $\left(\bar{\xi}_{N}^{0}(s)\right)_{0 \leq s \leq t}$ evolves just like $\left(\xi_{s}^{0}\right)_{0 \leq s \leq t}$, which allows us to bound $\mathbb{P}\left(\xi_{N}^{0}(t)=\emptyset\right)$, and we used (4.8) to bound $\mathbb{P}\left(E_{t}^{c}\right)$.

Finally we let

$$
a_{N}=\frac{1}{(2 N+1)^{1-\epsilon^{\prime 2}}}-\frac{2}{2 N+1}
$$

and

$$
b_{N}=\frac{2 N+1}{2 K \log (2 N+1)}
$$

so that the last bound can be written $\left(1-a_{N}\right)^{b_{N}}$. Then

$$
\left(1-a_{N}\right)^{b_{N}}=e^{b_{N} \log \left(1-a_{N}\right)} \leq e^{-b_{N} a_{N}},
$$

and since $a_{N} b_{N} \underset{N \rightarrow \infty}{\longrightarrow} \infty$, it follows that $e^{-b_{N} a_{N}}$ goes to 0 as $N$ goes to $\infty$.

\subsubsection{Proof of Proposition 4.1.3}

The last step consists in showing that the same convergence holds for the expectation. In order to do so we shall recall a classical result from the theory of probability about the convergence of a collection of random variable 


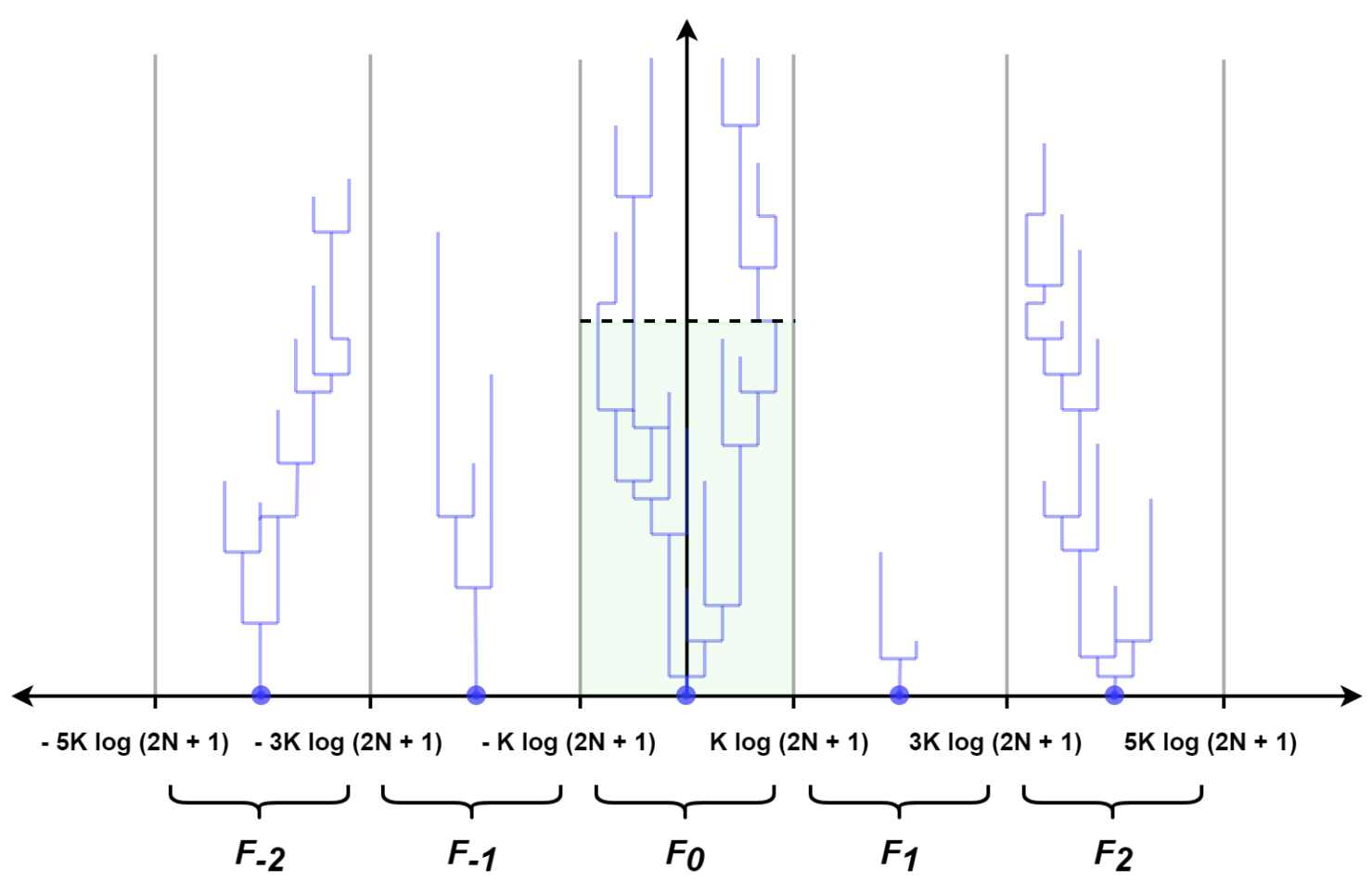

Figure 4.1: The construction of the current section, represented in the space-time diagram introduced in the previous chapter. the horizontal axis represents the lattice $\mathbb{Z}$ and the vertical axis represents the time. The blue points are the elements of the configuration $A_{N}$, while the blue lines represent the valid paths in the graphical construction in a realization of the modified process $\left(\bar{\xi}_{N}^{0}(t)\right)_{t>0}$, starting from these points. The vertical gray lines represents the particles that are not allowed to spike in our construction, acting as hermetic walls in the space-time diagram. The green area represent the portion of the diagram in which the event $E_{t}$ is satisfied.

Definition 4.2.1 A collection of random variable $\left(Y_{i}\right)_{i \in I}$, where $I$ is some countable or uncountable set of indexes, is said to be uniformly integrable if the following holds

$$
\lim _{M \rightarrow \infty}\left(\sup _{i \in I} \mathbb{E}\left(\left|Y_{i}\right| \mathbb{1}_{\left\{\left|Y_{i}\right|>M\right\}}\right)\right)=0
$$

Now it is a well-known fact that if a sequence of random variables $\left(Y_{n}\right)_{n \in \mathbb{N}}$ converges in probability to some random variable $Y$, it doesn't necessarily implies that $\mathbb{E}\left(X_{n}\right) \underset{n \rightarrow \infty}{\longrightarrow} \mathbb{E}(X)$. Nonetheless this implication holds true with the additional assumption that the sequence is uniformly integrable. In fact we have the following theorem.

Theorem 4.2.2 If $Y_{n} \underset{n \rightarrow \infty}{\stackrel{\mathbb{P}}{\longrightarrow}} Y$, then the following are equivalent:

(i) $\left\{Y_{n}, n \geq 0\right\}$ is uniformly integrable,

(ii) $\mathbb{E}\left(Y_{n}\right) \underset{n \rightarrow \infty}{\longrightarrow} \mathbb{E}(Y)<\infty$. 
Proof: See Theorem 5.5.2 in [DUR10].

It is therefore sufficient to show that $\left(\tau_{N} / \log (2 N+1)\right)_{N \in \mathbb{N}^{*}}$ is uniformly integrable, and the result will then follow from Proposition 4.1.2.

For some $M>0$ and some $N \in \mathbb{N}^{*}$ it is easy to see that we have the following

$$
\mathbb{E}\left(\frac{\tau_{N}}{\log (2 N+1)} \mathbb{1}_{\left\{\frac{\tau_{N}}{\log (2 N+1)}>M\right\}}\right)=\int_{0}^{\infty} \mathbb{P}\left(\frac{\tau_{N}}{\log (2 N+1)}>\max (t, M)\right) d t .
$$

Now using inequality (4.6) and the previously proven fact that $C^{\prime}>0$ when $\gamma>1$ we have the following

$$
\begin{aligned}
& \int_{0}^{\infty} \mathbb{P}\left(\frac{\tau_{N}}{\log (2 N+1)}>\max (t, M)\right) d t \\
& =\int_{0}^{M} \mathbb{P}\left(\frac{\tau_{N}}{\log (2 N+1)}>M\right) d t+\int_{M}^{\infty} \mathbb{P}\left(\frac{\tau_{N}}{\log (2 N+1)}>t\right) d t \\
& \leq(2 N+1)\left[\int_{0}^{M} e^{-C^{\prime} \log (2 N+1) M} d t+\int_{M}^{\infty} e^{-C^{\prime} \log (2 N+1) t} d t\right] \\
& =(2 N+1)^{1-C^{\prime} M}\left[M+\frac{1}{C^{\prime} \log (2 N+1)}\right],
\end{aligned}
$$

where $C^{\prime}$ is the same constant as in the previous proof. Without loss of generality we assume that $M>\frac{1}{C^{\prime}}$, so that the bound above is decreasing in $N$, from what we get

$$
\sup _{n \in \mathbb{N}^{*}} \mathbb{E}\left(\frac{\tau_{N}}{\log (2 N+1)} \mathbb{1}_{\left\{\frac{\tau_{N}}{\log (2 N+1)}>M\right\}}\right) \leq 3^{1-C^{\prime} M}\left[M+\frac{1}{C^{\prime} \log (3)}\right]
$$

Finally the right-hand side of inequality (4.9) goes to 0 when $M$ goes to $\infty$, which ends the proof. 


\section{Chapter 5}

\section{The complete graph process}

\subsection{Metastability in the complete graph process}

In this chapter, we're aimed to study the process defined on the complete graph of size $N$. Notice that in the complete graph setting it doesn't really make sense to consider an infinite process anymore, as any neuron would be activated by a spike infinitely many times in any time interval for example, moreover the minimal conditions for the existence of an infinitesimal generator are not satisfied. Luckily, unlike in chapter 3, we won't need any infinite process in the course of the proof.

For some $N \in \mathbb{N}$ we let $S_{N}^{\prime}=\llbracket 1, N \rrbracket$ and for any $i \in S_{N}^{\prime}$ we let $\mathbb{V}_{N, i}^{\prime}=V_{N}^{\prime} \backslash\{i\}$. Now write $\left(\zeta_{N}(t)\right)_{t \geq 0}$ for the equivalent of the finite lattice process $\left(\xi_{N}(t)\right)_{t \geq 0}$ in this complete graph setting, that is to say the process obtained when $S_{N}$ is replaced by $S_{N}^{\prime}$ and $\mathbb{V}_{N}$ by $\mathbb{V}_{N}^{\prime}$. This is the interacting particle system taking value in $\{0,1\}^{N}$ which dynamic is characterized by the following generator

$$
\mathscr{L}_{N}^{c} f(\zeta)=\gamma \sum_{i \in \mathbb{Z}}\left(f\left(\pi_{N, i}^{\dagger, c}(\zeta)\right)-f(\zeta)\right)+\sum_{i \in \mathbb{Z}} \zeta_{i}\left(f\left(\pi_{N, i}^{c}(\zeta)\right)-f(\zeta)\right)
$$

where the $\pi_{N, i}^{\dagger, c}$ 's and $\pi_{N, i}^{c}$ 's are maps from $\{0,1\}^{N}$ to $\{0,1\}^{N}, \pi_{N, i}^{\dagger, c}$ being defined as usual (see (3.2)), and $\pi_{N, i}^{c}$ being defined for any $i \in \llbracket 1, N \rrbracket$ as follows:

$$
\left(\pi_{N, i}^{c}(\zeta)\right)_{j}= \begin{cases}0 & \text { if } j=i \\ \max \left(\zeta_{i}, \zeta_{j}\right) & \text { if } j \neq i\end{cases}
$$

The existence and uniqueness of the process are guaranteed by the finiteness of the state space (see Theorem 3.9 in [LIG85])). In this instantiation every neuron is connected to one another so that when a single active neuron spikes, every other neuron becomes active.

Let $\sigma_{N}$ denote the time of extinction of this finite process,

$$
\sigma_{N} \stackrel{\text { def }}{=} \inf \left\{t \geq 0: \zeta_{N}(t)_{i}=0 \text { for any } i \in \llbracket 1, N \rrbracket\right\} .
$$


The result we're aimed to prove is that for the complete graph instantiation the result of convergence of the renormalized time of extinction toward an exponential random variable that was proven in the lattice case for small $\gamma$ now holds for any positive $\gamma$. This is the object of the following theorem.

Theorem 5.1.1 For any $\gamma>0$ the following convergence holds

$$
\frac{\sigma_{N}}{\mathbb{E}\left(\sigma_{N}\right)} \underset{N \rightarrow \infty}{\stackrel{\mathscr{D}}{\longrightarrow}} \mathscr{E}(1)
$$

\subsection{Proof of Theorem 5.1.1}

We adopt the same conventions as in the previous chapters regarding the notation, identifying $\{0,1\} \llbracket 1, N \rrbracket$ with $\mathscr{P}(\llbracket 1, N \rrbracket)$ and indicating the initial state as a superscript when it is different from the whole space $\llbracket 1, N \rrbracket$. This convention applies to the time of extinction as well.

The Markov process $\left(\zeta_{N}(t)\right)_{t \geq 0}$ has three communicating classes, which are visited in increasing order. The first one contains only the "all one" state, which is left definitively after the first spike/leak, and the third one contains only the "all zero" state, which is an absorbing state. The second class contains all the other states. As the absorbing state is accessible from the second class, standard results on irreducibility tell us that the time of extinction $\sigma_{N}$ is almost surely finite. It implies that $\mathbb{P}\left(\sigma_{N}>t\right)$ converges to 0 as $t$ diverges.

Moreover it is clear from the definition of $\left(\zeta_{N}(t)\right)_{t \geq 0}$ that $\mathbb{P}\left(\sigma_{N}>t\right)$ is a continuous and decreasing function of $t$. Therefore we can define $\lambda_{N}$ to be the unique value in $\mathbb{R}^{+}$satisfying the following equality

$$
\mathbb{P}\left(\sigma_{N}>\lambda_{N}\right)=e^{-1}
$$

The proof of Theorem 5.1.1 follows the same general line as the proof of Theorem 3.1.1. The main ingredient of the proof is the Proposition 5.2.1 below, which is the equivalent of Proposition 3.18 in the complete setting, but is proven using entirely different techniques. Theorem 5.1.1 then follows from Corollary 5.2.2 and Proposition 5.2.3, which tell us respectively that $\sigma_{N} / \lambda_{N}$ converges in distribution to an exponential random variable of mean 1 and that $\mathbb{E}\left(\sigma_{N}\right) \underset{N \infty}{\sim} \lambda_{N}$.

Proposition 5.2.1 For any $\gamma>0$ and for any $s, t \geq 0$ the following holds

$$
\lim _{N \rightarrow \infty}\left|\mathbb{P}\left(\frac{\sigma_{N}}{\lambda_{N}}>s+t\right)-\mathbb{P}\left(\frac{\sigma_{N}}{\lambda_{N}}>s\right) \mathbb{P}\left(\frac{\sigma_{N}}{\lambda_{N}}>t\right)\right|=0 .
$$

Proof: In our complete graph setting there is no spatial dependency between the neurons like in the lattice setting, so that the law of the time of extinction is impacted by the initial 
state only through its cardinal. In particular, for any $1 \leq k \leq N$ and any $A \in \mathscr{P}(\llbracket 1, N \rrbracket)$ of size $k, \sigma_{N}^{A}$ has the same law as $\sigma_{N}^{\llbracket 1, k \rrbracket}$. Using the Markov property and this last remark we have

$$
\begin{aligned}
& \left|\mathbb{P}\left(\frac{\sigma_{N}}{\lambda_{N}}>s+t\right)-\mathbb{P}\left(\frac{\sigma_{N}}{\lambda_{N}}>s\right) \mathbb{P}\left(\frac{\sigma_{N}}{\lambda_{N}}>t\right)\right| \\
& =\sum_{k=1}^{N}\left|\mathbb{P}\left(\frac{\sigma_{N}^{\llbracket 1, k \rrbracket}}{\lambda_{N}}>t\right)-\mathbb{P}\left(\frac{\sigma_{N}}{\lambda_{N}}>t\right)\right| \cdot \mathbb{P}\left(\left|\zeta_{N}\left(\lambda_{N} s\right)\right|=k\right) \\
& \leq \sum_{k=1}^{\left\lfloor\frac{N}{2}\right\rfloor} \mathbb{P}\left(\left|\zeta_{N}\left(\lambda_{N} s\right)\right|=k\right)+\sum_{k=\left\lceil\frac{N}{2}\right\rceil}^{N}\left|\mathbb{P}\left(\frac{\sigma_{N}^{\llbracket 1, k \rrbracket}}{\lambda_{N}}>t\right)-\mathbb{P}\left(\frac{\sigma_{N}}{\lambda_{N}}>t\right)\right|
\end{aligned}
$$

We fix $\epsilon>0$. In order to prove the desired result we show that we can find $N$ big enough such that

$$
\sum_{k=\left\lceil\frac{N}{2}\right\rceil}^{N}\left|\mathbb{P}\left(\frac{\sigma_{N}^{\llbracket 1, k \rrbracket}}{\lambda_{N}}>t\right)-\mathbb{P}\left(\frac{\sigma_{N}}{\lambda_{N}}>t\right)\right|<\epsilon,
$$

and

$$
\sum_{k=1}^{\left\lfloor\frac{N}{2}\right\rfloor} \mathbb{P}\left(\left|\zeta_{N}\left(\lambda_{N} s\right)\right|=k\right)<\epsilon
$$

We start with (5.4), which is the easiest part. For any $\left\lceil\frac{N}{2}\right\rceil \leq k \leq N$ we denote by $E_{k}$ the event in which every active neuron in the process starting from $\llbracket 1, k \rrbracket$ becomes quiescent before any of them is affected by a spike. Notice that on the complementary event $E_{k}^{c}$ there is a spike affecting the process at some point, and that the process starting from $\llbracket 1, k \rrbracket$ and the process starting from $\llbracket 1, N \rrbracket$ become equal at this point (both will be in the state $\llbracket 1, N \rrbracket \backslash\{i\}$, where $i$ is the neuron that just spiked). It follows that we have

$$
\mathbb{P}\left(\frac{\sigma_{N}^{\llbracket 1, k \rrbracket}}{\lambda_{N}}>t \mid E_{k}^{c}\right)=\mathbb{P}\left(\frac{\sigma_{N}}{\lambda_{N}}>t \mid E_{k}^{c}\right) .
$$

From this we get that, for any $\left\lceil\frac{N}{2}\right\rceil \leq k \leq N$, 


$$
\begin{aligned}
& \left|\mathbb{P}\left(\frac{\sigma_{N}^{\llbracket 1, k \rrbracket}}{\lambda_{N}}>t\right)-\mathbb{P}\left(\frac{\sigma_{N}}{\lambda_{N}}>t\right)\right| \\
& =\left|\mathbb{P}\left(\frac{\sigma_{N}^{\llbracket 1, k \rrbracket}}{\lambda_{N}}>t \mid E_{k}\right)-\mathbb{P}\left(\frac{\sigma_{N}}{\lambda_{N}}>t \mid E_{k}\right)\right| \cdot \mathbb{P}\left(E_{k}\right) \\
& \leq \mathbb{P}\left(E_{k}\right) .
\end{aligned}
$$

Now since $\mathbb{P}\left(E_{k}\right)=\left(\frac{\gamma}{1+\gamma}\right)^{k} \leq\left(\frac{\gamma}{1+\gamma}\right)^{\frac{N}{2}}$ for any $k \geq\left\lceil\frac{N}{2}\right\rceil$ we get the following bound

$$
\sum_{k=\left\lceil\frac{N}{2}\right\rceil}^{N}\left|\mathbb{P}\left(\frac{\sigma_{N}^{\llbracket 1, k \rrbracket}}{\lambda_{N}}>t\right)-\mathbb{P}\left(\frac{\sigma_{N}}{\lambda_{N}}>t\right)\right| \leq \frac{N}{2}\left(\frac{\gamma}{1+\gamma}\right)^{\frac{N}{2}}
$$

which goes to zero as $N$ goes to infinity, so that we can find some $N_{1}$ such that (5.4) is satisfied for any $N \geq N_{1}$.

We now turn to (5.5). We will use a coupling. Let $\left(\widetilde{\zeta}_{N}(t)\right)_{t \geq 0}$ be the process defined as follows. For $t<\sigma_{N}$ the process is simply equal to $\zeta_{N}(t)$. Now suppose $i \in \llbracket 1, N \rrbracket$ is the last neuron active in $\left(\zeta_{N}(t)\right)_{t \geq 0}$ before the extinction at $\sigma_{N}$, then when $i$ leaks in $\left(\zeta_{N}(t)\right)_{t \geq 0}$, instead of leaking in $\left(\widetilde{\zeta}_{N}(t)\right)_{t \geq 0}$ it spikes. Then the dynamic of $\left(\widetilde{\zeta}_{N}(t)\right)_{t \geq 0}$ is the same as the dynamic of the process $\left(\zeta_{N}(t)\right)_{t \geq 0}$ with the only difference that whenever there is only one neuron remaining it doesn't leak, and spike at rate $1+\gamma$, so that there is no extinction for this stochastic process. The reason for asking that the last neuron spike at rate $1+\gamma$ instead of simply 1 is that if $X$ and $Y$ are two independent random variable exponentially distributed with rate 1 and $\gamma$ respectively, then one can easily compute that the law of $X$ conditioned on $X \leq Y$ is an exponential distribution of rate $1+\gamma$. Now for any $1 \leq k \leq\left\lfloor\frac{N}{2}\right\rfloor$, we have

$$
\begin{aligned}
\mathbb{P}\left(\left|\zeta_{N}\left(\lambda_{N} s\right)\right|=k\right) & =\mathbb{P}\left(\left|\zeta_{N}\left(\lambda_{N} s\right)\right|=k \mid \sigma_{N}>\lambda_{N} s\right) \mathbb{P}\left(\sigma_{N}>\lambda_{N} s\right) \\
& =\mathbb{P}\left(\left|\widetilde{\zeta}_{N}\left(\lambda_{N} s\right)\right|=k \mid \sigma_{N}>\lambda_{N} s\right) \mathbb{P}\left(\sigma_{N}>\lambda_{N} s\right) \\
& \leq \mathbb{P}\left(\left|\widetilde{\zeta}_{N}\left(\lambda_{N} s\right)\right|=k\right)
\end{aligned}
$$

so that it will be sufficient to prove that for $N$ big enough we have

$$
\sum_{k=1}^{\left\lfloor\frac{N}{2}\right\rfloor} \mathbb{P}\left(\left|\widetilde{\zeta}_{N}\left(\lambda_{N} s\right)\right|=k\right)<\epsilon
$$

One can easily see that the process $\left(\left|\widetilde{\zeta}_{N}(t)\right|\right)_{t \geq 0}$, that is to say the process counting the number of particles at any time $t$, is a Markov jump process taking value in $\llbracket 1, N \rrbracket$, which 
transition diagram is given in Figure 5.1.

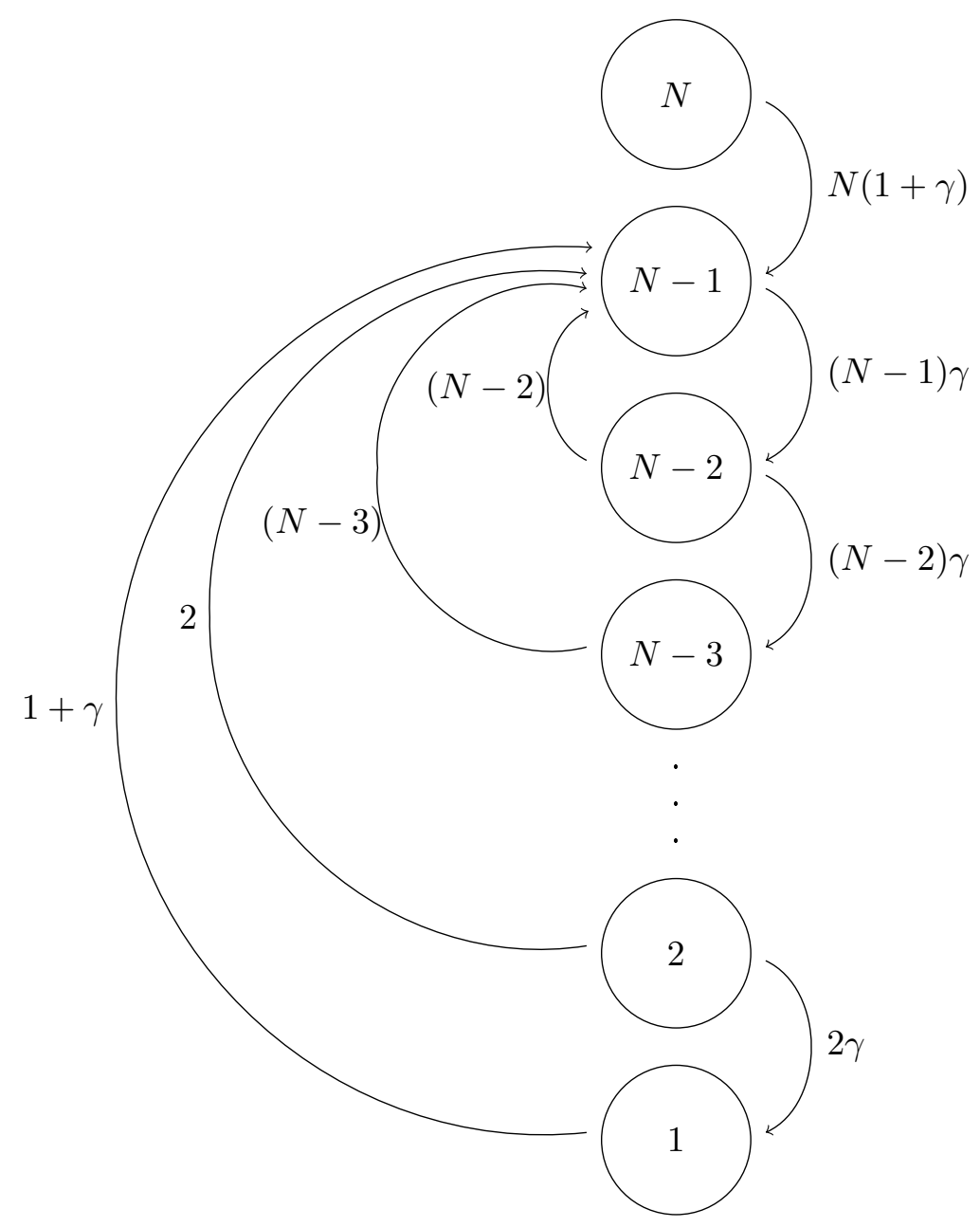

Figure 5.1: The transition diagram of the Markov jump process $\left(\widetilde{\zeta}_{N}(t)\right)_{t \geq 0}$.

We would like to compute an invariant measure for this chain, so that we need to solve the following equation for $\mu$.

$$
\mu Q=0
$$

where $Q$ is the transition intensities matrix, given by

$$
Q=\left(\begin{array}{ccccccc}
-(1+\gamma) & 0 & 0 & \cdots & 0 & 1+\gamma & 0 \\
2 \gamma & -2(1+\gamma) & 0 & \cdots & 0 & 2 & 0 \\
0 & 3 \gamma & -3(1+\gamma) & \cdots & 0 & 3 & 0 \\
\vdots & \vdots & \vdots & \cdots & \vdots & \vdots & \vdots \\
0 & 0 & 0 & \cdots & -(N-2)(1+\gamma) & N-2 & 0 \\
0 & 0 & 0 & \cdots & (N-1) \gamma & -(N-1) \gamma & 0 \\
0 & 0 & 0 & \cdots & 0 & N(1+\gamma) & -N(1+\gamma)
\end{array}\right) .
$$


This is the same as the following system of linear equations.

$$
\left\{\begin{array}{l}
-(1+\gamma) \mu_{1}+2 \gamma \mu_{2}=0, \\
-2(1+\gamma) \mu_{2}+3 \gamma \mu_{3}=0, \\
\quad \vdots \\
-(N-2)(1+\gamma) \mu_{N-2}+(N-1) \gamma \mu_{N-1}=0, \\
(1+\gamma) \mu_{1}+2 \mu_{2}+3 \mu_{3}+\ldots+(N-2) \mu_{N-2}-(N-1) \gamma \mu_{N-1}+N(1+\gamma) \mu_{N}=0, \\
-N(1+\gamma) \mu_{N}=0 .
\end{array}\right.
$$

Solving the system from top to bottom, we get that, for any $n \in \llbracket 2, N-1 \rrbracket$

$$
\mu_{n}=\frac{(1+\gamma)^{n-1}}{n \gamma^{n-1}} \mu_{1}
$$

Moreover $\mu_{N}=0$. Now from (5.9) and from the fact that the elements of $\mu$ need to sum up to 1 we get

$$
\mu_{1}=\left(\sum_{n=1}^{N-1} \frac{(1+\gamma)^{n-1}}{n \gamma^{n-1}}\right)^{-1}
$$

Disregarding all terms in the sum but the last one we obtain the following bound

$$
\mu_{1}<N\left(\frac{1+\gamma}{\gamma}\right)^{2-N}
$$

Hence, for any $1 \leq k \leq\left\lfloor\frac{N}{2}\right\rfloor$

$$
\mu_{k}<N\left(\frac{1+\gamma}{\gamma}\right)^{k+1-N}
$$

As a consequence,

$$
\sum_{k=1}^{\left\lfloor\frac{N}{2}\right\rfloor} \mu_{k}<\frac{N^{2}}{2}\left(\frac{1+\gamma}{\gamma}\right)^{1-\frac{N}{2}}
$$

Let $\left(\widetilde{\zeta}_{N}^{\mu}(t)\right)_{t>0}$ denote the process which initial state is chosen according to the invariant measure $\mu$. By this we mean that a value $k \in \llbracket 1, N \rrbracket$ is sorted according to the invariant measure, and that the process then start from the initial state $\llbracket 1, k \rrbracket$. For any $t \geq 0$ we have 
the following inequality:

$$
\mu_{N-1} \sum_{k=1}^{\left\lfloor\frac{N}{2}\right\rfloor} \mathbb{P}\left(\left|\widetilde{\zeta}_{N}^{\llbracket 1, N-1 \rrbracket}(t)\right|=k\right) \leq \sum_{k=1}^{\left\lfloor\frac{N}{2}\right\rfloor} \mathbb{P}\left(\left|\widetilde{\zeta}_{N}^{\mu}(t)\right|=k\right) .
$$

This last inequality gets us closer to our goal but it is still not exactly what we need as the left hand side involves the process starting from $\llbracket 1, N-1 \rrbracket$ while we would like it to start from the full initial configuration $\llbracket 1, N \rrbracket$. This little problem is solved as follows. Let $T_{N}$ be the time of the first jump of the process $\left(\widetilde{\zeta}_{N}(t)\right)_{t \geq 0}$, that is to say

$$
T_{N} \stackrel{\text { def }}{=} \inf \left\{t \geq 0:\left|\widetilde{\zeta}_{N}(t)\right| \neq N\right\} .
$$

Then, for any $t \geq 0$ and $1 \leq k \leq\left\lfloor\frac{N}{2}\right\rfloor$, the following holds

$$
\mathbb{P}\left(\left|\widetilde{\zeta}_{N}(t)\right|=k\right)=\mathbb{P}\left(\left|\widetilde{\zeta}_{N}^{\llbracket 1, N-1 \rrbracket}\left(\left(t-T_{N}\right)^{+}\right)\right|=k\right)
$$

where $\left(t-T_{N}\right)^{+}$stands for $\max \left(0, t-T_{N}\right)$. This last inequality is obtained from Markov property and the fact that whenever $T_{N}>t$ the events we look at are both of probability 0 for the $k$ we consider (assuming that $N \geq 3$ ).

Now from (5.10), (5.11) and (5.12), we obtain

$$
\begin{aligned}
\sum_{k=1}^{\left\lfloor\frac{N}{2}\right\rfloor} \mathbb{P}\left(\left|\widetilde{\zeta}_{N}\left(\lambda_{N} s\right)\right|=k\right) & =\sum_{k=1}^{\left\lfloor\frac{N}{2}\right\rfloor} \mathbb{P}\left(\left|\widetilde{\zeta}_{N}^{\llbracket 1, N-1 \rrbracket}\left(\left(\lambda_{N} s-T_{N}\right)^{+}\right)\right|=k\right) \\
& \leq \frac{1}{\mu_{N-1}} \sum_{k=1}^{\left\lfloor\frac{N}{2}\right\rfloor} \mathbb{P}\left(\left|\widetilde{\zeta}_{N}^{\mu}\left(\left(\lambda_{N} s-T_{N}\right)^{+}\right)\right|=k\right) \\
& \leq \frac{1}{\mu_{N-1}} \frac{N^{2}}{2}\left(\frac{1+\gamma}{\gamma}\right)^{1-\frac{N}{2}} .
\end{aligned}
$$

Moreover from the penultimate line of (5.8) we get

$$
\mu_{N-1}>\frac{\mu_{1}+\mu_{2}+\mu_{3}+\ldots+\mu_{N-2}}{\gamma(N-1)} .
$$

Furthermore, assuming that $N$ is sufficiently big for $\gamma(N-1)$ to be greater than one, and using again the fact that the elements of $\mu$ need to sum up to 1 , we obtain

$$
\mu_{N-1}+\frac{\mu_{1}+\mu_{2}+\mu_{3}+\ldots+\mu_{N-2}}{\gamma(N-1)} \geq \frac{1}{\gamma(N-1)}
$$


Combining the two previous equations we have

$$
\mu_{N-1} \geq \frac{1}{2 \gamma(N-1)}
$$

Hence, we finally get

$$
\begin{aligned}
\sum_{k=1}^{\left\lfloor\frac{N}{2}\right\rfloor} \mathbb{P}\left(\left|\widetilde{\zeta}_{N}\left(\lambda_{N} s\right)\right|=k\right) & \leq 2 \gamma(N-1) \frac{N^{2}}{2}\left(\frac{1+\gamma}{\gamma}\right)^{1-\frac{N}{2}} \\
& \leq(1+\gamma) N^{3}\left(\frac{1+\gamma}{\gamma}\right)^{-\frac{N}{2}}
\end{aligned}
$$

And the last bound goes to zero as $N$ goes to infinity, so that we can find some $N_{2}$ such that (5.5) is satisfied for any $N \geq N_{2}$.

Finally (5.4) and (5.5) are both satisfied for any $N$ greater than $\max \left(N_{1}, N_{2}\right)$ so that the proof is over.

From Proposition 5.2.1 we obtain the following corollary.

Corollary 5.2.2 For any $\gamma>0$ the following convergence holds

$$
\frac{\sigma_{N}}{\lambda_{N}} \underset{N \rightarrow \infty}{\stackrel{\mathscr{D}}{\longrightarrow}} \mathscr{E}(1) .
$$

Proof: The proof, which relies on a density argument and on the monotonicity of the function $t \mapsto \mathbb{P}\left(\frac{\sigma_{N}}{\lambda_{N}}>t\right)$, is exactly identical to the proof of Corollary 3.7.2.

It only remains to prove that we can replace $\lambda_{N}$ by $\mathbb{E}\left(\sigma_{N}\right)$ in Corollary 5.2.2. It follows from the proposition below.

Proposition 5.2.3 For any $\gamma>0$ the following convergence holds

$$
\lim _{N \rightarrow \infty} \frac{\mathbb{E}\left(\sigma_{N}\right)}{\lambda_{N}}=1
$$

Proof: From the following identity,

$$
\begin{aligned}
& {\left[\mathbb{P}\left(\frac{\sigma_{N}}{\lambda_{N}}>s+t\right)-\mathbb{P}\left(\frac{\sigma_{N}}{\lambda_{N}}>s\right) \mathbb{P}\left(\frac{\sigma_{N}}{\lambda_{N}}>t\right)\right]} \\
& =\sum_{k=1}^{N}\left[\mathbb{P}\left(\frac{\sigma_{N}^{\llbracket 1, k \rrbracket}}{\lambda_{N}}>t\right)-\mathbb{P}\left(\frac{\sigma_{N}}{\lambda_{N}}>t\right)\right] \cdot \mathbb{P}\left(\left|\zeta_{N}\left(\lambda_{N} s\right)\right|=k\right),
\end{aligned}
$$


and from the fact that for any $t \geq 0$ and $k \in \llbracket 1, N \rrbracket$ we have

$$
\mathbb{P}\left(\frac{\sigma_{N}^{\llbracket 1, k \rrbracket}}{\lambda_{N}}>t\right) \leq \mathbb{P}\left(\frac{\sigma_{N}}{\lambda_{N}}>t\right),
$$

it follows that

$$
\mathbb{P}\left(\frac{\sigma_{N}}{\lambda_{N}}>s+t\right) \leq \mathbb{P}\left(\frac{\sigma_{N}}{\lambda_{N}}>s\right) \mathbb{P}\left(\frac{\sigma_{N}}{\lambda_{N}}>t\right)
$$

Moreover from the definition of $\lambda_{N}$ we have that for any $n \in \mathbb{N}$

$$
\mathbb{P}\left(\frac{\tau_{N}}{\lambda_{N}}>n\right) \leq e^{-n}
$$

Therefore, for any $t \geq 0$ we have

$$
\mathbb{P}\left(\frac{\tau_{N}}{\lambda_{N}}>t\right) \leq e^{-\lfloor t\rfloor}
$$

Using the Dominated Convergence Theorem and (5.2.2) we finally get

$$
\begin{aligned}
\lim _{N \rightarrow \infty} \frac{\mathbb{E}\left(\tau_{N}\right)}{\lambda_{N}} & =\lim _{N \rightarrow \infty} \int_{0}^{\infty} \mathbb{P}\left(\frac{\tau_{N}}{\lambda_{N}}>t\right) d t \\
& =\int_{0}^{\infty} \lim _{N \rightarrow \infty} \mathbb{P}\left(\frac{\tau_{N}}{\lambda_{N}}>t\right) d t \\
& =\int_{0}^{\infty} e^{-t} d t \\
& =1 .
\end{aligned}
$$

And the proof is over. 


\section{Chapter 6}

\section{Conclusion and perspectives}

Throughout this thesis, we've stated and proven a series of results that suggest an interesting dichotomy with respect to metastability between loosely connected graph, such as the one-dimensional lattice with nearest-neighbors interaction, and highly connected graphs, such as the complete graph. In the first case the system is metastable only for small values of $\gamma$, while in the second case the system is metastable for any value of $\gamma$. This dichotomy is of course mostly theoretical, as the results we obtained are asymptotical while actual neural network are obviously finite. We can intuitively see that this difference comes from the fact that in the complete graph setting, the connectivity of the graph grows with the number of neurons $N$, raising the effect of spikes as well, so that the effect of leaks is proportionally diminished. At some point the spikes get the advantage, making a series of fatal leaks less probable and therefore setting the stage for metastability to arise ${ }^{1}$.

Yet simulations show that the exponential approximation is actually quite good for relatively small networks (see Figure 1.2 and Figure 1.3 for the one dimensional lattice case), as a matter of fact $N=100$ is already way sufficient to observe a metastable behavior in the lattice setting for $\gamma<0.4$ as well as in the complete graph setting for any reasonable $\gamma$ (i.e. of the same order of magnitude as the spiking rate). Knowing that the number of neurons in actual biological neural networks varies from few hundreds [WSTB86] to billions [LAAMP12], it means that if the parameter are chosen wisely, metastability can be observed in our model for any realistic size. It also suggests that, if we assume that neural networks need to behave in a metastable way in order to process information efficiently, it forces a delicate equilibrium between their different parameters (i.e. tendency to spike, tendency to leak, connectivity and size of the network), which would be interesting to investigate empirically.

The actual organization of the neurons in the cortex is notoriously complicated, and a full comprehension of the underlying structures of the brain are still out of reach from the neuroscience community (we refer to [SPO11] for a general review on this topic). While very specific networks, such as the retina neurons, actually present an interaction structure of the one-dimensional lattice type [BS98], it is questionable whether any networks could be wired

\footnotetext{
${ }^{1}$ This by the way highlight the fact that, while we defined our process to depend only one the parameter, the rate $\gamma$ corresponding to the leaks, letting the spike rate be equal to 1 , the only thing that really matters is actually their ratio.
} 
in a complete fashion. Yet, in the family of connected graphs, the one-dimensional lattice and the complete graph are at the two opposite side of the spectrum, which let us think that, since the metastability holds for both of them, any graph in between should behave in a metastable fashion provided that suitable values are chosen for the parameters. A possible continuation fo this work would therefore be to investigate these graphs in between. May be the one that come to mind most naturally would be the tree. The one-dimensional lattice is actually the regular tree of minimal degree, so that some of the techniques applicable in the lattice might be generalized to regular trees of any degree without too much complications. From here we might be able to prove metastability for any (non-regular) tree.

Moreover it is now well-known that the structure of the brain is not hard-wired (a feature called synaptic plasticity), so that the most interesting graphs we might consider are actually random graphs. The first we might think of is the Erdös-Rényi graph [ER59]. Indeed it is well-known that the super-critical ER graph is locally like a tree, so that if we are able to prove the metastability in the tree it is unlikely that we are not able to prove it for ER graph. Other random graphs of interest, often considered as good candidates for biological neural networks in the neuroscientific literature, are Power-law graphs (see for example [GL05]) and Small-world graphs (see [BB06]). A technical problem which might arise on our way is the question of how to define a sequence of random graphs in a meaningful way, so that we can formulate the possible metastability of the process in terms of a convergence of the time of extinction. While it was trivial for the non-random graph we worked with, it might not be so simple in the case of a random graph.

An other natural question to ask is whether similar results can be obtained for other closely or not so closely related models. The first option that one might consider is to work on the same model but with a different activation function $\phi$. Indeed, the definition of the model as it is given in Section 1.2 include an activation function, that was then kind of forgotten in the rest of this thesis as we always assumed that $\phi$ was the indicator function $\mathbb{1}_{x>0}$. This choice is mathematically convenient as it turns our model into an interacting particle systems, which allows us to use an already well developed literature to serve our purposes, but it is not the most realistic from a biological point of view. Linear or sigmoid functions could be interesting candidates for $\phi$.

The second option is to consider other models related to the original Galves-Löcherbach model. As the author is writing these lines a pre-print [LM20] has been self-archived on arxiv.org, addressing metastability for a version of the Galves-Löcherbach model in which the leakage effect is modeled by a continuous decrease of some fixed rate, with an interaction of the complete setting type. This last work nonetheless, as well as our, still relies on simplifying assumptions such that the homogeneity of the synaptic strength (a spike affects every post-synaptic neurons equally), the absence of inhibitory neurons (a spike never results in a decrease of membrane potential for the post-synaptic neurons), and the absence of electrical synapses, a mechanism induces by so-called gap-junction channels which results in a continuous sharing of membrane potential, and therefore tends to push neural networks toward their average membrane potential value. The next step would be to relax these assumptions in order to study metastability in models of higher complexity, considering models such as the one presented in [DO14] for example, and to try to determine, if metastability arises, for which values of the various parameters it does so. 


\section{Appendix A}

\section{Theory of duality}

We briefly recall the main results about duality for interacting particles systems. All the proof are omitted, and they can be found in [BG77]. In the following $S$ is any countable set.

Definition A.0.1 A map $\pi: \mathscr{P}(S) \mapsto \mathscr{P}(S)$ is called additive if for all $j \in S$, and for all $A \in \mathscr{P}(S)$ we have

$$
\pi(A)=\bigcup_{i \in A} \pi(\{i\})
$$

We define the notion of dual maps.

Definition A.0.2 Let $\pi: \mathscr{P}(S) \mapsto \mathscr{P}(S)$ and $\pi^{\prime}: \mathscr{P}(S) \mapsto \mathscr{P}(S)$. These maps are called dual if for any sets $A, B \in \mathscr{P}(S)$ we have the following

$$
\mathbb{1}_{\pi(A) \cap B}=\mathbb{1}_{A \cap \pi^{\prime}(B)} .
$$

We have the following result

Proposition A.0.3 Let $\pi: \mathscr{P}(S) \mapsto \mathscr{P}(S)$. A necessary and sufficient condition for the existence of a dual map $\pi^{\prime}$ (that is a map satisfying (A.1)) is for $\pi$ to be additive. This dual map is unique and is given by the following equations

$$
\text { for all } i \in S, \pi^{\prime}(\{i\})=\{j \in S: i \in \pi(\{j\})\} \text {, }
$$

and

$$
\text { for any } A \in \mathscr{P}(S), \pi^{\prime}(A)=\bigcup_{i \in A} \pi^{\prime}(\{i\})
$$

Now we define $\Sigma$ to be the set of applications $\pi$ from $\mathscr{P}(S)$ to $\mathscr{P}(S)$ satisfying the following properties 
1. $\pi$ is additive,

2. $\pi(\{i\})$ is a finite set for any $i \in S$,

3. $\{i \in S: \pi(\{i\}) \neq\{i\}\}$ is a finite set for any $i \in S$ as well.

Remark A.0.4 It is easy to verify that $\pi \in \Sigma$ if and only if $\pi^{\prime} \in \Sigma$.

For some application $\lambda: \Sigma \mapsto \mathbb{R}_{+}$we also define the two following infinitesimal generators. Let $\mathscr{L}$ and $\mathscr{L}^{\prime}$ be defined for any cylinder function $f$ by

$$
\mathscr{L} f=\sum_{\pi \in \Sigma} \lambda(\pi)(f \circ \pi-f) \text { and } \mathscr{L}^{\prime} f=\sum_{\pi \in \Sigma} \lambda(\pi)\left(f \circ \pi^{\prime}-f\right) .
$$

For any $i \in S$ we also define the following set

$$
I_{i}=\{\pi \in \Sigma: \pi(\{i\}) \neq\{i\}\} .
$$

Moreover we define the following quantities

$$
\alpha=\sup _{i \in S} \sum_{\pi \in I_{i}} \lambda(\pi) \text { and } \alpha^{\prime}=\sup _{i \in S} \sum_{\pi: \pi^{\prime} \in I_{i}} \lambda(\pi)
$$

as well as

$$
\beta=\sup _{i \in S} \sum_{\pi \in \Sigma} \lambda(\pi)(|\pi(\{i\})|-1) \text { and } \beta^{\prime}=\sup _{i \in S} \sum_{\pi \in \Sigma} \lambda(\pi)\left(\left|\pi^{\prime}(\{i\})\right|-1\right) .
$$

Now we have the following central result

Theorem A.0.5 Assume that $\alpha, \alpha^{\prime}, \beta, \beta^{\prime}<\infty$. Then for any $A \in \mathscr{P}(S)$ there exists two Markov processes $\left(\xi^{A}(t)\right)_{t \geq 0}$ and $\left(\xi^{\prime A}(t)\right)_{t \geq 0}$ on $\mathscr{P}(S)$ with initial state $A$ and having generator $\mathscr{L}$ and $\mathscr{L}^{\prime}$ respectively. These processes are additive in the sense that

$$
\xi^{A}(t)=\bigcup_{i \in A} \xi^{i}(t) \text { and } \xi^{\prime A}(t)=\bigcup_{i \in A} \xi^{i}(t)
$$

Moreover, they verify the duality property, that is, for any $A, B \in \mathscr{P}(S)$ such that at least one is finite, we have

$$
\mathbb{P}\left(\xi^{A}(t) \cap B \neq \emptyset\right)=\mathbb{P}\left(\xi^{B}(t) \cap A \neq \emptyset\right) .
$$




\section{Appendix B}

\section{Some usefull results}

\section{B.1 A measure determining set of functions}

It was claimed in Section 3.5.1 that the set of continuous and increasing function on $\{0,1\}^{\mathbb{Z}}$ is measure determining. While it seems to be considered as a somewhat classical result in the literature, we were unable to find a proof anywhere so that we wrote our own.

Proposition B.1.1 Let $\mu_{1}$ and $\mu_{2}$ be two probability measures on the measurable space $\left(\{0,1\}^{\mathbb{Z}}, \mathscr{B}\left(\{0,1\}^{\mathbb{Z}}\right)\right)$, where $\mathscr{B}\left(\{0,1\}^{\mathbb{Z}}\right)$ denotes the borelian $\sigma$-algebra on $\{0,1\}^{\mathbb{Z}}$. Suppose that for any $\left.\mathscr{B}\left(\{0,1\}^{\mathbb{Z}}\right)\right)$-measurable, continuous and increasing function $f:\{0,1\}^{\mathbb{Z}} \mapsto \mathbb{R}$ we have the following equality

$$
\int f d \mu_{1}=\int f d \mu_{2}
$$

then $\mu_{1}=\mu_{2}$.

Proof: Fix an arbitrary $B \in \mathscr{B}\left(\{0,1\}^{\mathbb{Z}}\right)$ different from the empty set. For any $n \geq 0$ define the function $f_{n}$ on $\{0,1\}^{\mathbb{Z}}$ by

$$
f_{n}(x)=\mathbb{1}\left\{x_{[-n, n]} \geq b_{[-n, n]} \text { for some } b \in B\right\},
$$

where for any $x \in\{0,1\}^{\mathbb{Z}}, x_{[-n, n]}$ is defined as follows

$$
x_{[-n, n]}(i)= \begin{cases}x(i) & \text { if } i \in[-n, n], \\ 0 & \text { otherwise. }\end{cases}
$$

The function $f_{n}$ can be written as follows 


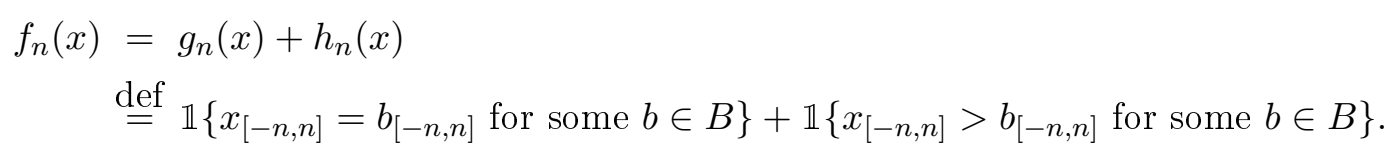

Notice that $f_{n}$ is a continuous and increasing function, so that by hypothesis we have

$$
\int f_{n} d \mu_{1}=\int f_{n} d \mu_{2}
$$

which can be written

$$
\int g_{n} d \mu_{1}+\int h_{n} d \mu_{1}=\int g_{n} d \mu_{2}+\int h_{n} d \mu_{2} .
$$

Now $h_{n}$ is a continuous and increasing function as well, so that the two integrals involving $h_{n}$ simply cancel out in the previous equation, and we are left with

$$
\int g_{n} d \mu_{1}=\int g_{n} d \mu_{2}
$$

Finally we have $\lim _{n \rightarrow \infty} g_{n}(x)=\mathbb{1}\{x \in B\}$, so that by dominated convergence theorem

$$
\mu_{1}(B)=\lim _{n \rightarrow \infty} \int g_{n} d \mu_{1}=\lim _{n \rightarrow \infty} \int g_{n} d \mu_{2}=\mu_{2}(B),
$$

which ends the proof.

\section{B.2 Fekete lemma}

Fekete lemma was initially stated for subadditive sequences in [FEK23], it can be extended to locally bounded superadditive real functions as follows.

Lemma B.2.1 Let $f: \mathbb{R}^{+} \mapsto \mathbb{R}$ be a locally bounded function such that for any $s, t \geq 0$ the following holds

$$
f(s+t) \geq f(s)+f(t) .
$$

Then we have the following

$$
\lim _{t \rightarrow \infty} \frac{f(t)}{t}=\sup _{t>0} \frac{f(t)}{t} .
$$


Proof: Let fix some $s>0$. Then any $t \geq 0$ can be written $t=q(t) s+r(t)$, where $q(t)$ (the "quotient") is a non-negative integer and $r(t)$ (the "remaining") belongs to [0,s[. Iterating the super-additivity property we have $f(q(t) s) \geq q(t) f(s)$ so that

$$
f(t)=f(q(t) s+r(t)) \geq q(t) f(s)+f(r(t)) .
$$

Now, using the assumption that $f$ is locally bounded, we have

$$
\frac{q(t) f(s)+f(r(t))}{t} \underset{t \rightarrow \infty}{\longrightarrow} \frac{f(s)}{s}
$$

from what it follows that

$$
\liminf _{t \rightarrow \infty} \frac{f(t)}{t} \geq \frac{f(s)}{s}
$$

The inequality above being true for any $s>0$ we get

$$
\liminf _{t \rightarrow \infty} \frac{f(t)}{t} \geq \sup _{t>0} \frac{f(t)}{t}
$$

The result then follows from the inequality above together with the trivial inequality

$$
\sup _{t>0} \frac{f(t)}{t} \geq \limsup _{t \rightarrow \infty} \frac{f(t)}{t} \geq \liminf _{t \rightarrow \infty} \frac{f(t)}{t}
$$

\section{B.3 Continuous time branching process}

At time 0 there is a single individual. Two independent exponential random clocks of parameter $\lambda$ and $\gamma$ respectively are attached to this individual. If the rate $\gamma$ clock rings before the other one, then the individual dies. In the contrary case the individual is replaced by two other individuals. Every new individual gets his two own independent exponential clocks of parameter $\lambda$ and $\gamma$ and so on.

We denote by $\left(Z_{t}\right)_{t \geq 0}$ the process corresponding to the number of individuals of the population along the time. Note that by hypothesis we have $Z_{0}=1$. We have the following result, which give the explicit value of the expectation at any time $t \geq 0$.

Proposition B.3.1 For any $\gamma>0$, and for any $t \geq 0$, we have

$$
\mathbb{E}\left(Z_{t}\right)=e^{-(\gamma-\lambda) t}
$$

Proof: The proof, which only involves solving an ordinary differential equation, is the 
same as the proof of Theorem 1.1 in chapter 8 of [SCH14]. 


\section{Bibliography}

[BAK96] P. BAK. How Nature Works. Springer, New York, 1996. 5

[BB06] D. S. BASSETT and E. BULLMORE. Small-world brain networks. Neuroscientist, 6:512-523, 2006. 66

[BG77] F. BERTEIN and A. GALVES. Une classe de systèmes de particules stable par association. 41:73-85, 1977. 13, 22, 67

[BP03] J. M. BEGSGS and D. D. PLENZ. Neuronal avalanches in neocortical circuits. J. Neurosci., 23:11167-11177, 2003. 5

[BS98] V. BRAITENBERG and A. SCHÜZ. Cortex: Statistics and Geometry of Neural Connectivity. Springer-verlag, 2 edition, 1998. 65

[CCMDRB15] J. CHEVALLIER, M. J. CACERES, M M. DOUMIC, and P. REYNAUDBOURRET. Microscopic approach of a time elapsed neural model. Mathematical Models and Methods in Applied Sciences, 25:266-2719, 2015. 2

[CGL17] M. CASSANDRO, A. GALVES, and E. LÖCHERBACH. Information transmission and criticality in the contact process. Journal of Statistical Physics, 168:1180-1190, 2017. 5

[CGOV84] M. CASANDRO, A. GALVES, E. OLIVIERI, and M.E. VARES. Maximum likelihood analysis of spike trains of interacting nerve cells. 35:603-634, 1984. 5

[CSK88] E. S. CHORNOBOY, L.P. SCHRAMM, and A. F. KARR. Maximum likelihood identification of neural point process systems. Biological Cybernetics, 59:265-275, 1988. 2

[DKJR17] G. DECO, M. L. KRINGELBACH, V.K. JIRSA, and P. RITTER. The dynamics of resting fluctuations in the brain: metastability and its dynamical cortical core. Scientific Reports, 7, 2017. 6

[DL88] R. DURRETT and X.F. LIU. The contact process on a finite set. The Annals of Probability, 16:1158-1173, 1988. 48

[DO14] A. DUARTE and G. OST. A model for neural activity in the absence of external stimuli. Markov Processes And Related Fields, 22:37-52, 2014. 2, 66 
[DUR81] R. DURRETT. An introduction to infinite particle systems. 11:109-150, 1981. 25

[DUR10] R. DURRETT. Probability: Theory and Examples. Cambridge University Press, fourth edition edition, 2010. 29, 54

[ER59] P. ERDÖS and A. RÉNYI. On random graphs. Publicationes Mathematicae, 6:290-297, 1959. 66

[FEK23] M. FEKETE. Über die verteilung der wurzeln bei gewissen algebraischen gleichungen mit ganzzahligen koeffizienten. Mathematische Zeitschrift, 17:228249, 1923. 70

[FGGL18] P.A. FERRARI, A. GALVES, I. GRIGORESCU, and E. LÖCHERBACH. Phase transition for infinite systems of spiking neurons. 172:1564-1575, 2018. iii, v, 2, 4, 7, 10, 11, 13, 15, 17, 22, 23, 29, 30, 47

[FL16] N. FOURNIER and E. LÖCHERBACH. On a toy model of interacting neurons. Annales de l'Institut Henri Poincaré, 52:1844-1876, 2016. 2

[FMNS15] R. FERNANDEZ, F. MANZOT, F. R. NARDI, and E. SCOPPOLA. Asymptotically exponential hitting times and metastability: a pathwise approach without reversibility. 20:1-37, 2015. 5

[FUS73] J. M. FUSTER. Unit activity in prefrontal cortex during delayed-response performance: Neuronal correlates of transient memory. Journal of Neurophysiology, 36:61-78, 1973. 6

[GEP19] A. GALVES, E.LÖCHERBACH, and C. POUZAT. A system of interacting neurons with short term synaptic facilitation. Journal of Statistical Physics, 178:869-892, 2019. 2

[GK77] W. GERSTNER and WERNER KISTLER. Spiking Neuron Models: An Introduction. Cambridge University Press, 1977. 1

[GL05] G. GRINSTEIN and R. LINSKER. Synchronous neural activity in scale-free network models versus random network models. Proceedings of the National Academy of Science of the United States of America, 102:9948-9953, 2005. 66

[GL13] A. GALVES and E. LÖCHERBACH. Infinite systems of interacting chains with memory of variable length. 151:896-921, 2013. 2

[GL15] A. GALVES and E. LÖCHERBACH. Modeling networks of spiking neurons as interacting processes with memory of variable length. Journal de la Société Française de Statistique, 157:17-32, 2015. 2

[GRI80] D. GRIFFEATH. The basic contact processes. 11:151-185, 1980. 29

[HAR76] T.E. HARRIS. On a class of set valued markov processes. 4:175-194, 1976. 22 
[HAR78] T.E. HARRIS. Additive set-valued markov processes and graphical methods. 6:355-378, 1978. 19

[HAW71] A.G. HAWKES. Point spectra of some mutually exciting point processes. 33:438-443, 1971. 2

[HOL72] R. HOLLEY. Markovian interaction processes with finite range interaction. 43:1961-1967, 1972. 29

[HRBR15] N. R. HANSEN, P. REYNAUD-BOURET, and V. RIVOIRARD. Lasso and probabilistic inequalities for multivariate point processes. Bernoulli, 21(1):83-143, 2015. 2

[KV01] R. E. KASS and V. VENTURA. A spike-train probability model. Neural computation, 13(8):1713-1720, 2001. 2

[LAAMP12] R. LENT, F. A. C. AZEVEDO, C. H. ANDRADE-MORAES, and A. V. O. PINTO. How many neurons do you have? some dogmas of quantitative neuroscience under revision. European Journal of Neuroscience, 35(1):1-9, 2012. 65

[LIG85] T. M. LIGGETT. Interacting Particle Systems. Grundlehren der mathematischen Wissenschaften, 1985. 2, 4, 12, 25, 27, 37, 55

[LM20] E. LÖCHERBACH and P. MONMARCHÉ. Metastability for systems of interacting neurons. https://arxiv.org/abs/2004.13353v1, 2020. 66

[LP71] J.L. LEBOWITZ and O. PENROSE. Rigorous treatment of metastable states in the van der waals-maxwell theory. 3:211-236, 1971. 5

[MGLP15] A. DE MASI, A. GALVES, E. LÖCHERBACH, and E. PRESUTTI. Hydrodynamic limit for interacting neurons. Journal of Statistical Physics, 158:866-902, 2015. 2

[MOU93] T.S. MOUNTFORD. A metastable result for the finite multidimensional contact process. 36:216-226, 1993. 5

[OV05] E. OLIVIERI and M. E. VARES. Large Deviations and Metastability. Cambridge University Press, Vol. 100 of Encyclopedia of Mathematics and its Applications, 2005. 5

[POI08] H. POINCARÉ. L'invention mathématique. L'enseignement Mathématique, 10:357-369, 1908. 6

[RD10] E. T. ROLLS and G. DECO. The Noisy Brain: Stochastic Dynamics as a Principle of Brain Function. Cambridge University Press, 2010. 2

[SCH85] R.H. SCHONMANN. Metastability for the contact process. 41:445-464, 1985. 5

[SCH14] R.B. SCHINAZI. Classical and Spatial Stochastic Processes With Applications to Biology. Birkhäuser Basel, 2 edition, 2014. 72 
[SPO11] O. SPORNS. The non-random brain: Efficiency, economy and complex dynamics. Frontiers in Computational Neuroscience, 5(5), 2011. 65

[THO95] H. THORISSON. Coupling methods in probability theory. Scandinavian Journal of Statistics, 22:159-182, 1995. 19

[WER07] G. WERNER. Metastability, criticality and phase transitions in brain and its models. Biosystems, 90:496-3508, 2007. 4

[WSTB86] G. J. WHITE, E. SOUTHGATE, J.E. THOMSON, and S. BRENNER. The structure of the nervous sytem of the nematode caenorhabditis elegans. Philos Trans $R$ Soc Lond B Biol Sci., 314:1-340, 1986. 65 


\section{Index}

Activation function, 2, 3

Additivity, 20, 49, 67, 68

Birkhoff's ergodic theorem, 29

Branching process, 14, 15, 49, 71

Chemical synapse, 1

Contour agrument, 29

Coupling, 14, 15, 19, 30, 37, 49, 58

Critical value, $5,11,13,38$

Duality, 13, 14, 22-24, 26, 41, 67, 68

Ergodicity, 28, 39, 42

Extinction time, 7, 14, 18, 21, 22, 38, 47, 55,56

GL model, 2

Graph of interaction, 11

Graphical construction, 19, 20, 23, 25, 35

Interacting particle system, 2, 12, 17, 55

Invariant measure, 4, 5, 24, 26, 27, 59, 60

Extremal, 27, 35
Krein-Milman theorem, 27

Leakage, 1-4, 38

Markov

Process, 4, 39, 58, 68

Property, 57, 61

Membrane potential, 1, 3

Memorylessness, 5, 47

Metastability, 4-6, 18, 38, 56

Phase transition, 4, 11, 12, 23, 29

Point process, 2, 3

Poisson, 3, 19, 23, 32, 33, 51

Postsynaptic, 1, 3

Presynaptic

Neuron, 1, 11

Set, 3

Stochastic monotonicity, 25, 35, 42

Superadditivity, 48, 70

Uniform integrability, 53 